\title{
Environmental Surveillance Master Sampling Schedule
}

L. E. Bisping

February 1995

Prepared for the U.S. Department of Energy under Contract DE-AC06-76RLO 1830

Pacific Northwest Laboratory

Operated for the U.S. Department of Energy by Battelle Memorial Institute 


\title{
DISCLAIMER
}

This report was prepared as an account of work sponsored by an agency of the United States Government. Neither the United States Government nor any agency thereof, nor Battelle Memorial Institute, nor any of their employees, makes any warranty, expressed or implied, or assumes any legal liability or responsibility for the accuracy, completeness, or usefulness of any information, apparatus, product, or process disclosed, or represents that its use would not infringe privately owned rights. Reference herein to any specific commercial product, process, or service by trade name, trademark, manufacturer, or otherwise does not necessarily constitute or imply its endorsement, recommendation, or favoring by the United States Government or any agency thereof, or Battelle Memorial Institute. The views and opinions of authors expressed herein do not necessarily state or reflect those of the United States Government or any agency thereof.

\author{
PACIFIC NORTHWEST LABORATORY \\ operated by \\ BATTELLE MEMORIAL INSTITUTE \\ for the \\ UNITED STATES DEPARTMENT OF ENERGY \\ under Contract DE-ACO6-76RLO 1830
}

Printed in the United States of America

Available to DOE and DOE contractors from the

Office of Scientific and Technical Information, P.O. Box 62, Oak Ridge, TN 37831; prices available from (615) 576-8401. FTS 626-8401.

Available to the public from the National Technical Information Service, U.S. Department of Commerce, 5285 Port Royal Rd., Springfield, VA 22161. 


\section{DISCLAIMER}

Portions of this document may be illegible in electronic image products. Images are produced from the best available original document. 
ENVIRONMENTAL SURVEILLANCE

MASTER SAMPLING SCHEDULE

L. E. Bisping

February 1995

Prepared for

the U.S. Department of Energy

under Contract DE-AC06-76RLO 1830

Pacific Northwest Laboratory

Richland, Washington 99352 


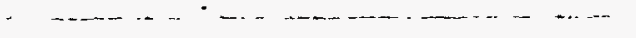


Environmental surveillance of the Hanford Site and surrounding areas is conducted by the Pacific Northwest Laboratory (PNL) ${ }^{(a)}$ for the U.S. Department of Energy (DOE). This document contains the planned 1994 schedules for routine collection of samples for the Surface Environmental Surveillance Project (SESP), Drinking Water Project, and Ground-Water Surveillance Project.

Samples are routinely collected for the SESP and analyzed to determine the quality of air, surface water, soil, sediment, wildlife, vegetation, foodstuffs, and farm products at Hanford Site and surrounding communities. In some cases, the location name is in an abbreviated form. This is due to the fact that the location name is exactly as it appears in the SESP database.

The responsibility for monitoring onsite drinking water falls outside the scope of the SESP. The Hanford Environmental Health Foundation is responsible for monitoring the nonradiological parameters as defined in the National Drinking Water Standards while PNL conducts the radiological monitoring of the onsite drinking water. PNL conducts the drinking water monitoring project concurrent with the SESP to promote efficiency and consistency, utilize expertise developed over the years, and reduce costs associated with management, procedure development, data management, quality control, and reporting.

The ground-water sampling schedule identifies ground-water sampling events used by PNL for environmental surveillance of the Hanford Site. Sampling is indicated as annual, semi-annual, quarterly, or monthly in the sampling schedule. Some samples are collected and analyzed as part of groundwater monitoring and characterization programs at Hanford (e.g. Resources Conservation and Recovery Act (RCRA), Comprehensive Environmental Response, Compensation, and Liability Act (CERCLA), or Operationa7). The number of samples planned by other programs are jdentified in the sampling schedule by a number in the analysis column. and a project designation in the Cosample column. Well sampling events may be merged to avoid redundancy in cases where sampling is planned by both environmental surveillance and another program. Thus a designation for a particular constituent to be collected by

(a) Pacific Northwest Laboratory is operated by Battelle Memorial Institute for the U.S. Department of Energy under Contract DE-AC06-76RLO 1830. 
environmental surveillance and by another program does not necessarily indicate that separate samples will be collected. The distribution of wells sampled by the different programs is shown in Section 2.0 of Part II, Well Distribution According to Program. The pocket at the end of the document includes a plate of the Monitoring Well Locations at the Hanford Site Washington. Additional detail on the rationale for ground-water sampling is presented in Ground-Water Sample Collection and Analysis Plan for the Hanford Site Ground-Water Surveillance Project, R. W. Bryce, J. C. Evans, and K. C. 01 sen, 1991, PNL-7872, Pacific Northwest Laboratory, Richland, Washington.

This schedule is subject to modification during the year in response to changes in site operations, program requirements, and the nature of the observed results. Operational limitations such as weather, mechanical failures, sample availability, etc., may also require schedule modifications. Changes will be documented in the respective project files, but this schedule will not be reissued and, therefore, may not be an accurate record of samples collected during the year.

The purpose of these monitoring projects is to evaluate levels of radioactive and nonradioactive pollutants in the Hanford environs, as required in DOE Order 5400.1, "General Environmental Protection Program," and DOE Order 5400.5, "Radiation Protection of the Public and the Environment." The sampling methods will be the same as those described in the Environmental Monitoring PIan. United States Department of Energy, Richland Field Office, DOE/RL 91-50, U.S. Department of Energy, Richland, Washington.

Environmental and ground-water samples that are split with the Washington State Department of Health are indicated in the schedule as are environmental samples that are split with the U.S. Food and Drug Administration.

Questions can be directed to Roger Dirkes, Manager, Surface Environmental Surveillance Project; Ernest Antonio, Manager, Drinking Water Monitoring Project; and Stuart Luttre11, Manager, Ground-Water Survei1lance Project. 


\section{CONTENTS}

SUMMARY

FIGURES

PART I. HANFORD SURFACE ENVIRONMENTAL SURVEILLANCE

1.0 AIR SURVEILLANCE $\ldots \ldots \ldots \ldots$

1.1 AIR - PARTICULATE FILTER . . . . . . . . 1

1.2 AIR - GASES AND VAPOR ............ 3

2.0 SURFACE-WATER SURVEILLANCE $\ldots \ldots \ldots$

2.1 WATER - COLUMBIA RIVER ........... 5

2.2 RIVERBANK SPRINGS ............. 6

2.3 ONSITE PONDS ......................... 6

2.4 OFFSITE WATER SYSTEMS $\ldots \ldots \ldots . \ldots$

2.5 ONSITE DRINKING WATER $\ldots \ldots \ldots \ldots$

3.0 FOODSTUFFS AND FARM PRODUCTS $\ldots \ldots \ldots$

3.1 WHOLE MILK .................. 9

3.2 POULTRY AND EGGS .................... 9

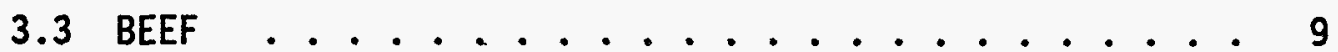

3.4 LEAFY VEGETABLES ............. 10

3.5 VegetABLES ................... 10

3.6 FRUIT $\ldots \ldots \ldots \ldots 11$

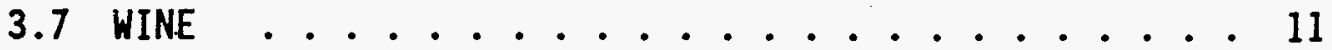

3.8 WHEAT AND ALFALFA $\ldots \ldots \ldots 12$

4.0 WILDLIFE ........................... 14

4.1 AqUATIC BIOTA .................. 14

4.2 GEESE .................... 15

4.3 GAME BIRDS .................. 15 


\section{CONTENTS (contd)}

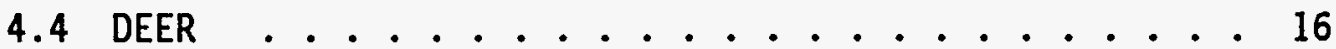

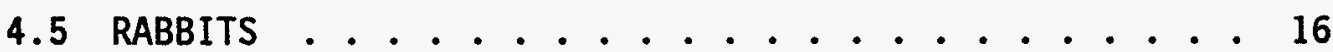

5.0 SOIL AND VEGETATION .................. 19

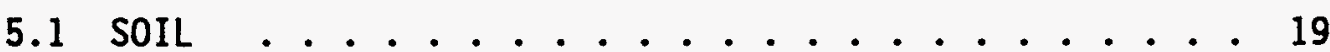

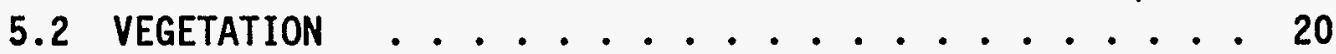

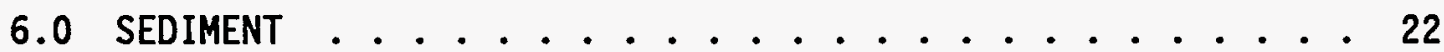

7.0 EXTERNAL RADIATION .................... 24

7.1 THERMOLUMINESCENT DOSIMETERS (TLDs) . . . . . 24

7.1.1 Terrestrial Locations ........... 24

7.1.2 Columbia River Shoreline Locations . . . . 28

7.2 COLUMBIA RIVER SHORELINE RADIATION SURVEYS . . . . 29

PART II. HANFORD GROUND-WATER SURVEILLANCE

1.0 HANFORD SITE WELLS ................ 33

2.0 WELL DISTRIBUTION ACCORDING TO PROGRAM ........ 53 


\section{FIGURES}

PART I. HANFORD SURFACE ENVIRONMENTAL SURVEILLANCE •

Figure 1.1 Air Sampling Locations ............. 4

Figure 2.1 Surface Water and Drinking Water Sampling Locations . 8

Figure 3.1 Food and Farm Product Sampling Locations . . . . . 13

Figure 4.1 Wildlife Sampling Areas ........... 18

Figure 5.1 Soil and Vegetation Sampling Locations ...... 21

Figure 6.1 Sediment Sampling Locations ........... 23

Figure 7.1 Thermoluminescent Dosimeter (TLD) Locations on the Hanford Site ............... 26

Figure 7.2 Thermoluminescent Dosimeter (TLD) Locations for Perimeter and Community Sites ......... 27

Figure 7.3 Thermoluminescent Dosimeter (TLD) Locations on the Hanford Reach of the Columbia River . . . . . . 30

PART II. HANFORD GROUND-HATER SURVEILLANCE

Figure 2.I Distribution of Wells Sampled by the Ground-Water Surveillance, CERCLA, and RCRA/Operational

Monitoring Programs ............. 53

Plate 1. Monitoring Hell Locations at the Hanford Site, Washington 


\section{ABBREVIATIONS}

\section{FREQUENCY SYMBOLS USED}

$\begin{array}{ll}\text { A } & \text { annually } \\ \text { BA } & \text { biannually (every } 2 \text { years) } \\ \text { BM } & \text { bimonthly (every } 2 \text { months) } \\ \text { BW } & \text { biweekly (every } 2 \text { weeks) } \\ \text { M } & \text { monthly } \\ \text { M Comp } & \text { monthly composite } \\ \text { Q } & \text { quarterly } \\ \text { Q Comp } & \text { quarterly composite } \\ \text { SA } & \text { semiannually } \\ \text { W } & \text { weekly }\end{array}$

Numbers The numbers in the ground-water tables indicate the estimated number of samples to be collected by other projects.

\section{ANALYSIS SYMBOLS USED}

Generally, standard elements, chemical, and isotope designations are used to indicate the analyses performed. Other analysis designations used are:
ATk
alkalinity
Alpha
total alpha activity of sample
Anions
major anions-generally $\mathrm{Cl}, \mathrm{F}, \mathrm{NO}_{2}, \mathrm{NO}_{3}, \mathrm{SO}_{4}$
Beta
total beta activity of sample
Comp. On7y
sample analyzed as part of composite only
DO
dissolved oxygen
DS
dissolved solids
Gamma Scan
analysis of photon energy spectrum for individual photon-emitting radionuc7ides, including ${ }^{60} \mathrm{Co},{ }^{65} \mathrm{Zn}$, ${ }^{106} \mathrm{Ru}$, and ${ }^{137} \mathrm{Cs}$
HTO
tritiated water $\left({ }^{3} \mathrm{H}^{1} \mathrm{H}^{16} \mathrm{O}\right)$
ICP-f
major metals by inductively coupled plasma spectrometry - samples filtered in the field
ICP-u
major metals by inductively coupled plasma spectrometry - samples unfiltered
Isotopic uranium
${ }^{234} \mathrm{U},{ }^{235} \mathrm{U},{ }^{238} \mathrm{U}$
Isotopic plutonium
${ }^{238} \mathrm{Pu},{ }^{239-240} \mathrm{Pu}$
LEP
Lo ${ }^{3} \mathrm{H}$
samples analyzed by Low Energy Photon System analytical procedure includes electrolytic enrichment 


\section{ABBRËVIATIONS (contd)}

NRA

SS

TOC

$\mathrm{U}$

VOA

WQ not routinely analyzed

suspended solids

total organic carbon

natural uranium

volatile organic analytes generally including carbon tetrachloride, chloroform, tetrachloroethylene, trichloroethylene, dichloroethylene, vinylchloride, benzene \& toluene, ethylbenzene, xylene

water quality - nonradiological

\section{NOTES/ABBREVIATIONS USED}

The Programs and Projects listed in the Cosample column of the Ground-Water Surveillance section are as follows:

\begin{tabular}{|c|c|}
\hline Cosample & Program \\
\hline $100-B C-5$ & CERCLA \\
\hline $100-F R-3$ & CERCLA \\
\hline $100-H R-3$ & CERCLA \\
\hline $100-K R-4$ & CERCLA \\
\hline $100-N R-2$ & CERCLA \\
\hline $200-B P-5$ & CERCLA \\
\hline 200-UP-1 & CERCLA \\
\hline $200-Z P-1$ & CERCLA \\
\hline $300-F F-5$ & CERCLA \\
\hline 300-FIRE & CERCLA \\
\hline ERDF & CERCLA \\
\hline OPER:200-E & Operational \\
\hline OPER:200-W & Operationa 7 \\
\hline OPER:222-S & Operational \\
\hline OPER: 400 & Operational \\
\hline OPER: 600 & Operational \\
\hline OPER:B-P1 ant & Operational \\
\hline OPER:B-POND & Operational \\
\hline OPER:Gable Mt. & Operational \\
\hline OPER:K & Operational \\
\hline OPER:PUREX & Operational \\
\hline OPER:U-14 & Operational \\
\hline
\end{tabular}
Project

$100-B C-5$

100-FR-3

100-HR-3

$100-K R-4$

$100-N R-2$

200-BP-5

200-UP-1

200-ZP-1

$300-F F-5$

300-Area Fire Station

Environmental Restoration Disposal Facility

200-East Area

200-West Area

222-S Building

400 Area

600 Area

B-Plant

B-Pond

Gable Mountain Pond

100-K Area

PUREX PJant

U-14 Crib 


\section{ABBREVIATIONS (contd)}

\begin{tabular}{|c|c|}
\hline Cosample & Progr \\
\hline OPER:U-Plant & Operationa \\
\hline OPER:U1/2 & Operation \\
\hline OPER:U14 & Operation \\
\hline OPER:U17 & Operation \\
\hline 100-D Pond & RCRA \\
\hline 1301 & RCRA \\
\hline 1324 & RCRA \\
\hline 1325 & RCRA \\
\hline $183-H$ & RCRA \\
\hline 2101-M POND & RCRA \\
\hline $216-A-10$ & RCRA \\
\hline $216-A-29$ & RCRA \\
\hline $216-A-36 B$ & RCRA \\
\hline $216-B-3$ & RCRA \\
\hline $216-B-63$ & RCRA \\
\hline $216-5-10$ & RCRA \\
\hline $216-U-12$ & RCRA \\
\hline 300-TRENCH & RCRA \\
\hline LERF & RCRA \\
\hline LLWMA-1 & RCRA \\
\hline LLWMA-2 & RCRA \\
\hline LLWMA-3 & RCRA \\
\hline LLWMA-4 & RCRA \\
\hline LLHMA-5 & RCRA \\
\hline NRDWL & RCRA \\
\hline SST-A-AX & RCRA \\
\hline SST-B-BY-BX & RCRA \\
\hline SST-C & RCRA \\
\hline$S S T-S-S X$ & RCRA \\
\hline SST-T & RCRA \\
\hline
\end{tabular}

Project

U-Plant

U-1/U-2 Cribs

U-14 Crib

U-17 Crib

100-D Pond

1301-N Liquid Waste Disposal Facility 1324-N/NA site

1325-N Liquid Waste Disposal Facility 183-H Solar Evaporation Basins

2101-M Pond

216-A-10 Crib

216-A-29 Ditch

216-A-36B Crib

216-B-3 Pond System

216-B-63 Trench

216-S-10 Pond and Ditch

216-U-12 Crib

300 Area Process Trenches

200-East Area Liquid Effluent

Retention Facility

Low-Lever Waste Management Area 1

Low-Lever Waste Management Area 2

Low-Lever Waste Management Area 3

Low-Lever Waste Management Area 4

Low-Lever Waste Management Area 5

Non-Radioactive Dangerous Waste Landfill

Single She11 Tank Waste Management Area A-AX

Single She11 Tank Waste Management Area B-BY-BX

Single Shell Tank Waste Management Area $C$

Single She 11 Tank Waste Management Area S-SX

Single She11 Tank Waste Management Area $\mathrm{T}$ 


\section{ABBREVIATIONS (contd)}

- Cosample

SST-TX-TY

SST-U

SWL

DOH
RCRA

Program

RCRA

RCRA

Washington State

Department of

Health

PORTABLE INSTRUMENT SYMBOLS USED

BICRON Microrem meter

GM Geiger-Maeller counter

PIC Pressurized Ionization Chamber

Single She11 Tank Waste Management Area TX-TY

Single Shel1 Tank Waste Management Area U

Solid Waste Landfill 
PART I. HANFORD SURFACE ENVIRONMENTAL SURVEILLANCE 


\subsection{AIR SURVEILLANCE}

\subsection{AIR - PARTICULATE FILTER}

Individual Samples

Cocation fre-

Number $(a)$ quency Analyses

\begin{tabular}{cc}
\multicolumn{3}{c}{ Composited Sample } \\
\hline $\begin{array}{c}\text { Composite } \\
\text { Group }\end{array}$ & $\begin{array}{c}\text { quency } \\
\text { qualyses }\end{array}$ \\
\hline
\end{tabular}

Onsite

$100 \mathrm{~K}$ Area

$100 N-1325$ Crib

100 D Area

$N$ of $200 E$

$E$ of $200 E$

200 ESE

$S$ of $200-E$

B Pond

$B$ Pond

Army Loop Camp

200 Tel. Exchange

200 H SE

200 W SE

300 Hater Intake

300 South Gate

300 Trench

$300 \mathrm{NE}$

$400 \mathrm{E}$

$400 \mathrm{H}$

$400 \mathrm{~S}$

$400 \mathrm{~N}$

Wye Barricade

Wye Barricade

Perimeter

Ringold Met Tower

$W$ End of Fir Rd

$H$ End of Fir Rd

Dogwood Met Tower

Dogwood Met Tower

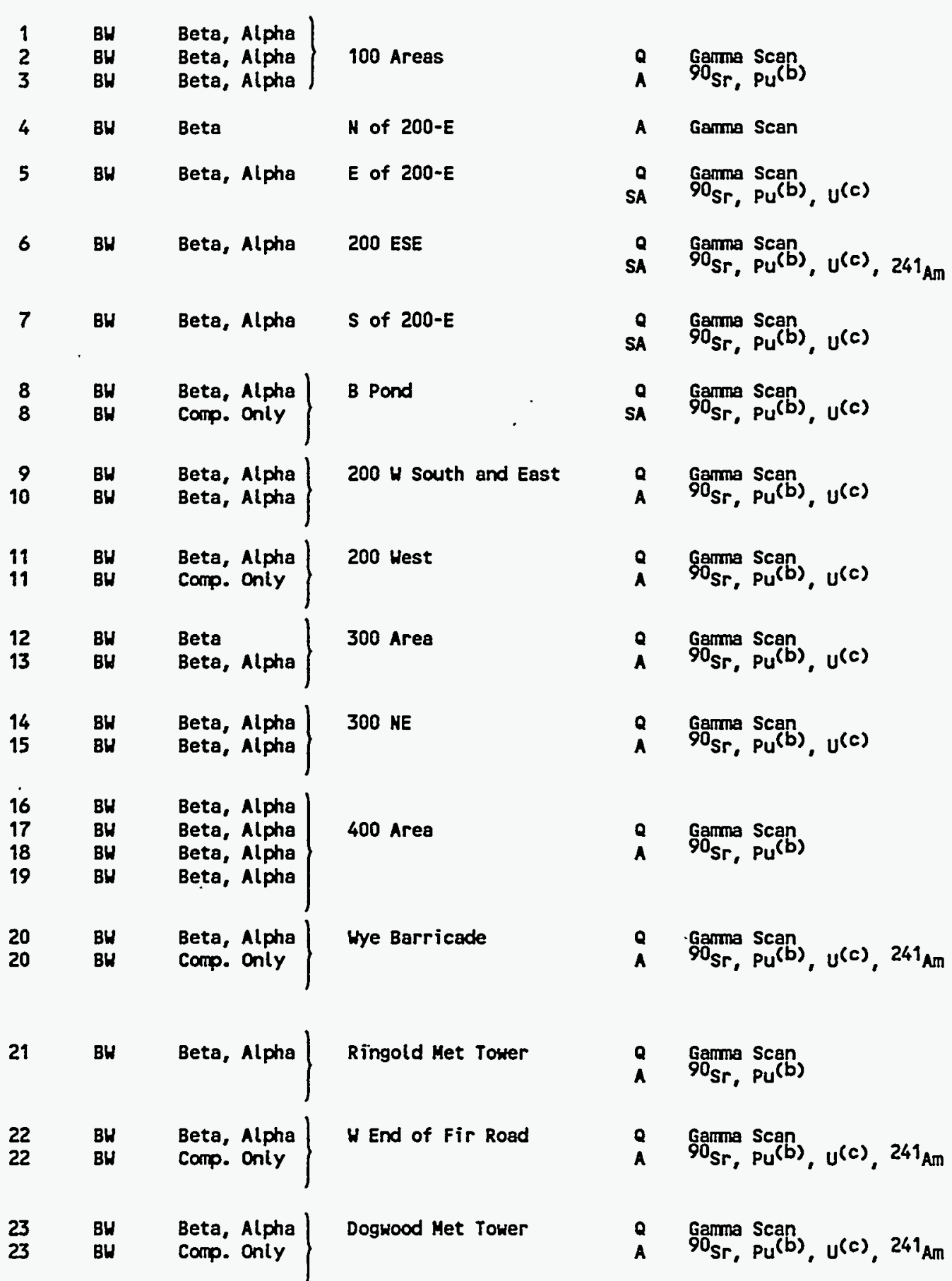




\subsection{AIR - PARTICULATE FILTER (contd)}

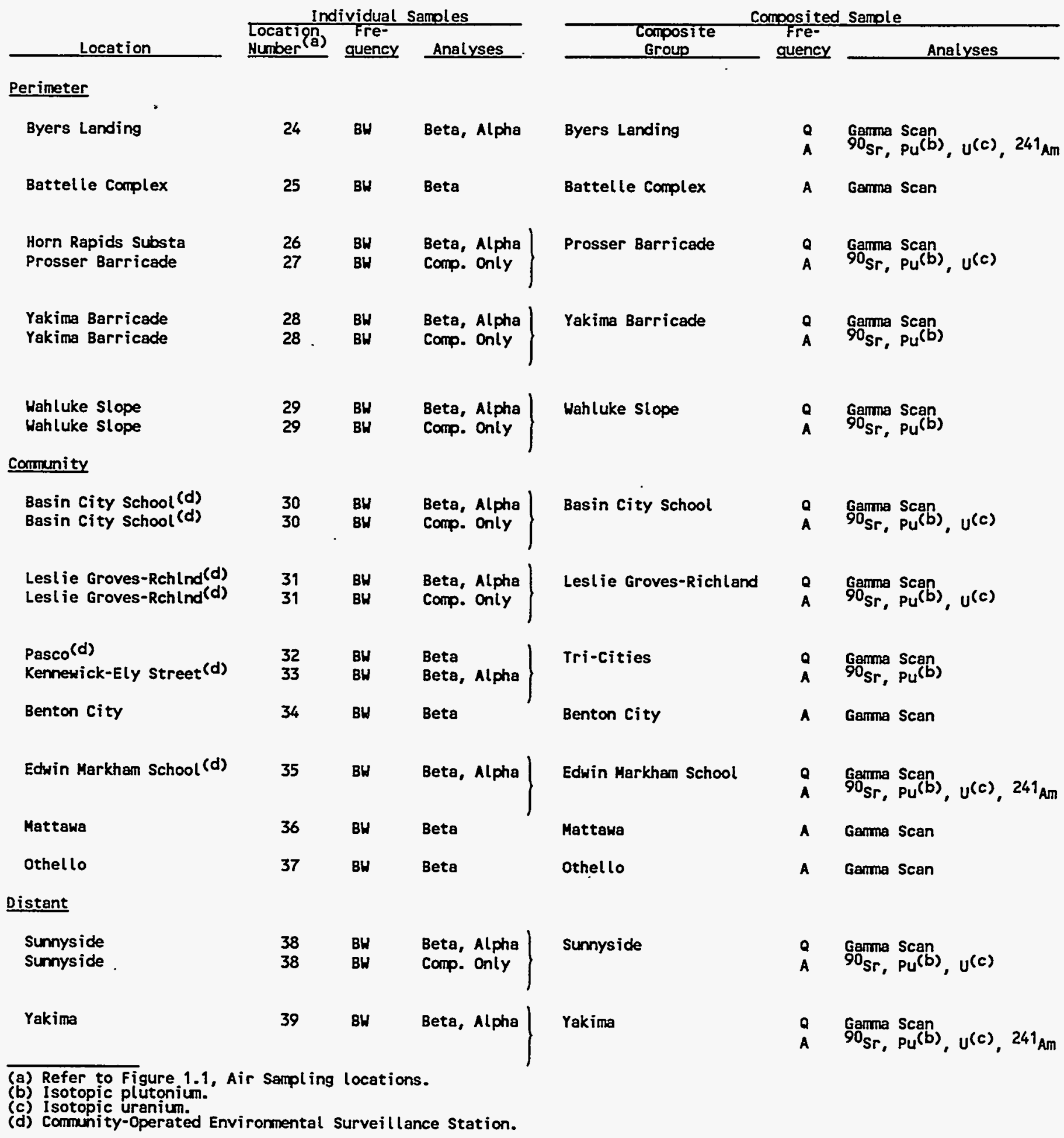


1.2 AIR - GASES AND VAPOR

Location

Location Fre-

guency Analyses

Fre-

quency ${ }^{(b)}$ Analyses

Fre-

Number

Onsite

$100 \mathrm{~K}$ Area
$100 \mathrm{~N}-1325 \mathrm{Crib}$

200 ESE

200 Tel. Exchange

300 South Gate

$300 \operatorname{Trench}^{(c)}$

$300 \mathrm{NE}$

$400 \mathrm{E}$

\section{Perimeter}

Ringold Met Tower Dogwood Met Tower Byers Landing Prosser Barricade

Hahluke Slope

1
2
6
10
13
14
15
16

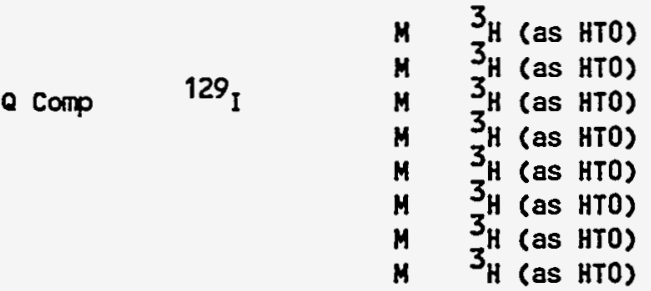

Community

Basin City School (d)

Leslie Groves-Rchlnd(d)

Edwin Markham School (d)

31

32

36

BH

$131_{\text {I }}$

a Comp

129 I

Q Comp $129_{\text {I }}$

BH 131,

Distant

Sunnyside

Yakima

38
39

BH $131_{1}$

a comp

$129_{1}$

$\begin{array}{ll}M & 3_{H} \text { (as HTO) } \\ H & 3_{H} \text { (as HTO) }\end{array}$

(a) Refer to Figure 1.1, Air Samoling locations.

(b) Samples are col lected monthly and composited for quarterly analyses.

(c) Tate it is collected and the second is archived for one month and then submitted to the analytical lab.

(d) Comminity-operated Enviromental Surveillance Station. 


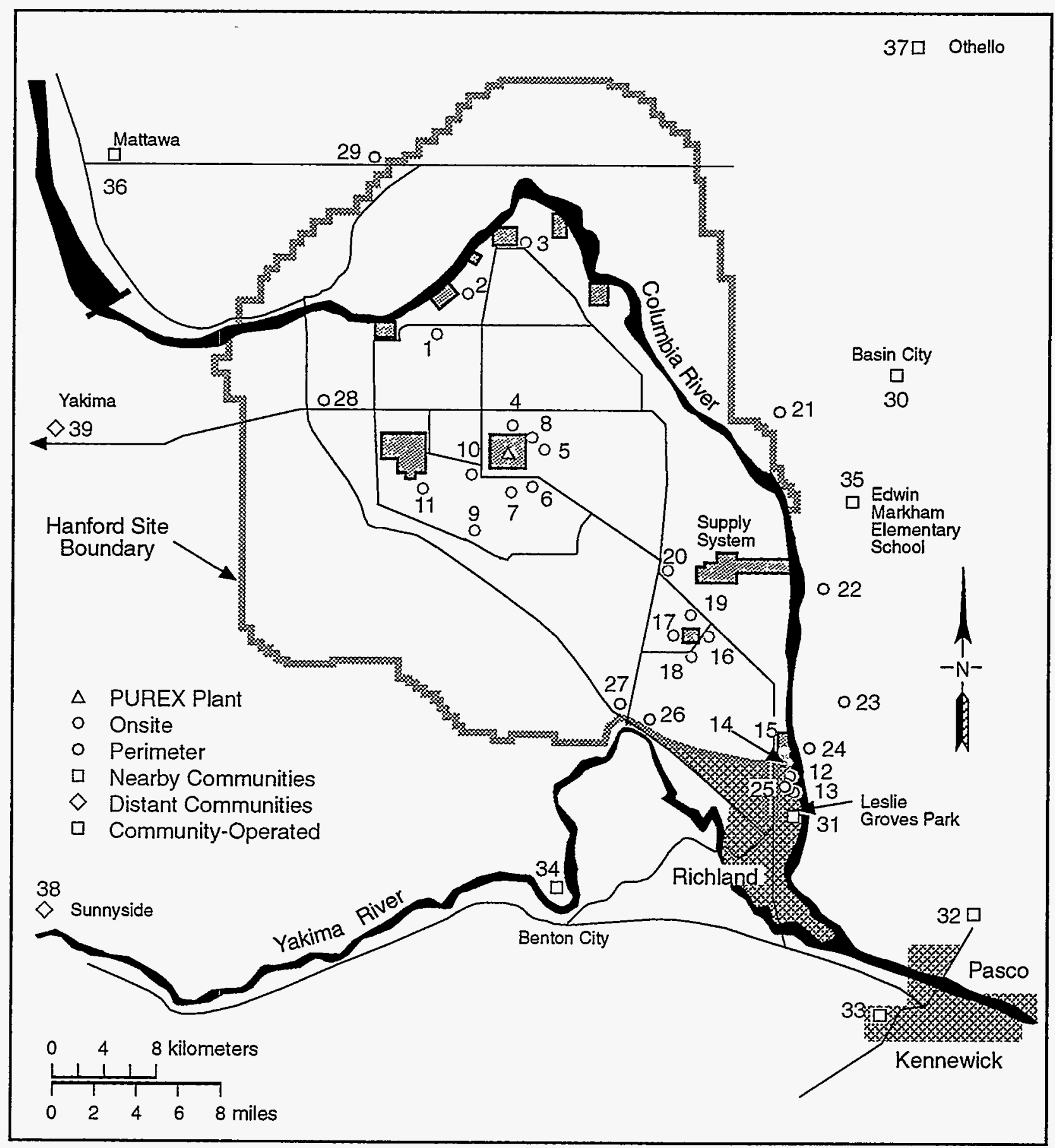

\$9412049.2

Figure 1.1. Air Sampling Locations 


\subsection{SURFACE-WATER SURVEILLANCE}

\subsection{WATER - COLUMBIA RIVER}

\begin{tabular}{|c|c|c|c|}
\hline Location $^{(a)}$ & Sample Type & Erequency & Analyses \\
\hline \multirow[t]{3}{*}{ Priest Rapids Dam } & Cumulative & M Comp(b) & $\begin{array}{l}\text { Alpha, Beta, Lo }{ }^{3} \mathrm{H}, \text { Gamma Scan, }{ }^{90} \mathrm{Sr},{ }^{99} \mathrm{TC} \text {, } \\
U^{(c)}, \text { DOH(d) }\end{array}$ \\
\hline & Particulate (filter) & $\begin{array}{l}\text { M Comp(e) } \\
\text { Q Comp }\end{array}$ & $\begin{array}{l}\text { Gama Scan } \\
\text { Pu(f) }\end{array}$ \\
\hline & Soluble (resin) & $\begin{array}{l}M \operatorname{comp}^{(e)} \\
Q \operatorname{comp}\end{array}$ & $\begin{array}{l}\text { Gamma Scan } \\
129_{I}, P_{U}(f)\end{array}$ \\
\hline \multirow[t]{2}{*}{ Vernita } & Grab & $\mathbf{Q}$ & $\begin{array}{l}\text { Wa-NASQAN }{ }^{(g)} \text { : Temp, DO, Turbidity, PH, Fecal } \\
\text { Coliform, SS, DS, Cond, Hardhess as CaCO3, } \\
\text { P, Cr, H-Kjeldahl, TOC, Fe, } \mathrm{HH}^{-\mathrm{H}}\end{array}$ \\
\hline & Grab (Transect) ${ }^{(h)}$ & a & $\begin{array}{l}\text { Lo }{ }^{3} H_{,},{ }^{90} \mathrm{Sr}, \mathrm{U}^{(\mathrm{C})} \text {, Volatile Organics, ICP-u, } \\
\text { Anions }\end{array}$ \\
\hline $100 \mathrm{H}$ & Grab (Transect) ${ }^{(i)}$ & A & $\begin{array}{l}\text { Alpha, Beta, Lo }{ }^{3} \mathrm{H},{ }^{90} \mathrm{Sr},{ }^{99} \mathrm{TC}, \mathrm{U}^{(\mathrm{C})} \text {, } \\
\text { Gamma Scan, Yolatile Organics, ICP-u, } \\
\text { Anions, DOH(j) }\end{array}$ \\
\hline $100 \mathrm{~F}$ & Grab (Transect) ${ }^{(i)}$ & 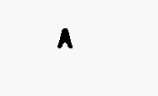 & $\begin{array}{l}\text { Lo }{ }^{3} \mathrm{H},{ }^{90} \mathrm{Sr}, \mathrm{U}^{(\mathrm{C})} \text {, Volatile Organics, ICP-U, } \\
\text { Anions }\end{array}$ \\
\hline Hanford Townsite & Grab (Transect) ${ }^{(i)}$ & A & $\begin{array}{l}\text { Lo }{ }^{3} \mathrm{H},{ }^{90} \mathrm{Sr},{ }^{99} \mathrm{TC}, \mathrm{U}^{(\mathrm{C})} \text {, Volatile Organics, } \\
\text { ICP-U, Anions }\end{array}$ \\
\hline 300 Area & Grab (Transect) $(i)$ & A & $\begin{array}{l}\text { Lo }{ }^{3} \mathrm{H},{ }^{90} \mathrm{Sr}, \mathrm{U}^{(\mathrm{C})} \text {, Volatile organics, ICP-U, } \\
\text { Anions }\end{array}$ \\
\hline \multirow[t]{6}{*}{ Richland Pump house } & Cumulative & $H$ & Alpha, Beta \\
\hline & Cumulative & M Comp $(b)$ & $\underset{U(\mathrm{C})}{\text { Alpha, Beta, Lo }}{ }^{3 \mathrm{H}}$, Gaima Scan, ${ }^{90} \mathrm{Sr},{ }^{99} \mathrm{TC}$, \\
\hline & Particulate (filter) & $\begin{array}{l}\text { M Comp(e) } \\
Q \text { Comp }\end{array}$ & $\begin{array}{l}\text { Gamma Scan } \\
\mathrm{Pu}(f)\end{array}$ \\
\hline & Soluble (resin) & $\begin{array}{l}\text { H Comp(e) } \\
\text { Q Comp }\end{array}$ & $\begin{array}{l}\text { Gamma Scan } \\
129_{1}, P_{u}(f)\end{array}$ \\
\hline & Grab (Transect) ${ }^{(i)}$ & Q & $\begin{array}{l}\text { Lo } 3_{H,} 90_{S r}, U^{(C)} \text {, Volatile Organics, ICP-u, } \\
\text { Anions }\end{array}$ \\
\hline & Grab & a & $\begin{array}{l}\text { Wh-HASQAN(g): Temp, DO, Turbidity, PH, Fecal } \\
\text { Coliform, SS, DS, Cond, Hardiness as CaCOz, } \\
\text { P, Cr, H-Kjeldahl, TOC, Fe, HH3-H }\end{array}$ \\
\hline
\end{tabular}

(a) Refer to Figure 2.1, Surface Water and Drinking Water Sampling Locations.

(b) Composite of veekly samples.

(c) Isotopic uranium.

(d) Duplicate samples with the Washington State Department of Health (January and June only).

(e) Composite of biweekly sample.

(f) Isotopic plutonium.

(g) Numerous water quality (UR) analyses are performed by the United States Geological Survey (USGS) in conjunction Hith the liational Stream Quality Accounting Network (HASQAK) Progran.

(h) 4 samples collected along cross-section.

(i) 10 samples collected along cross-section.

(j) Duplicate samples $w i$ ih the Washington State Department of Health collected at three sample sites along transect. 


\subsection{RIVERBANK SPRINGS}

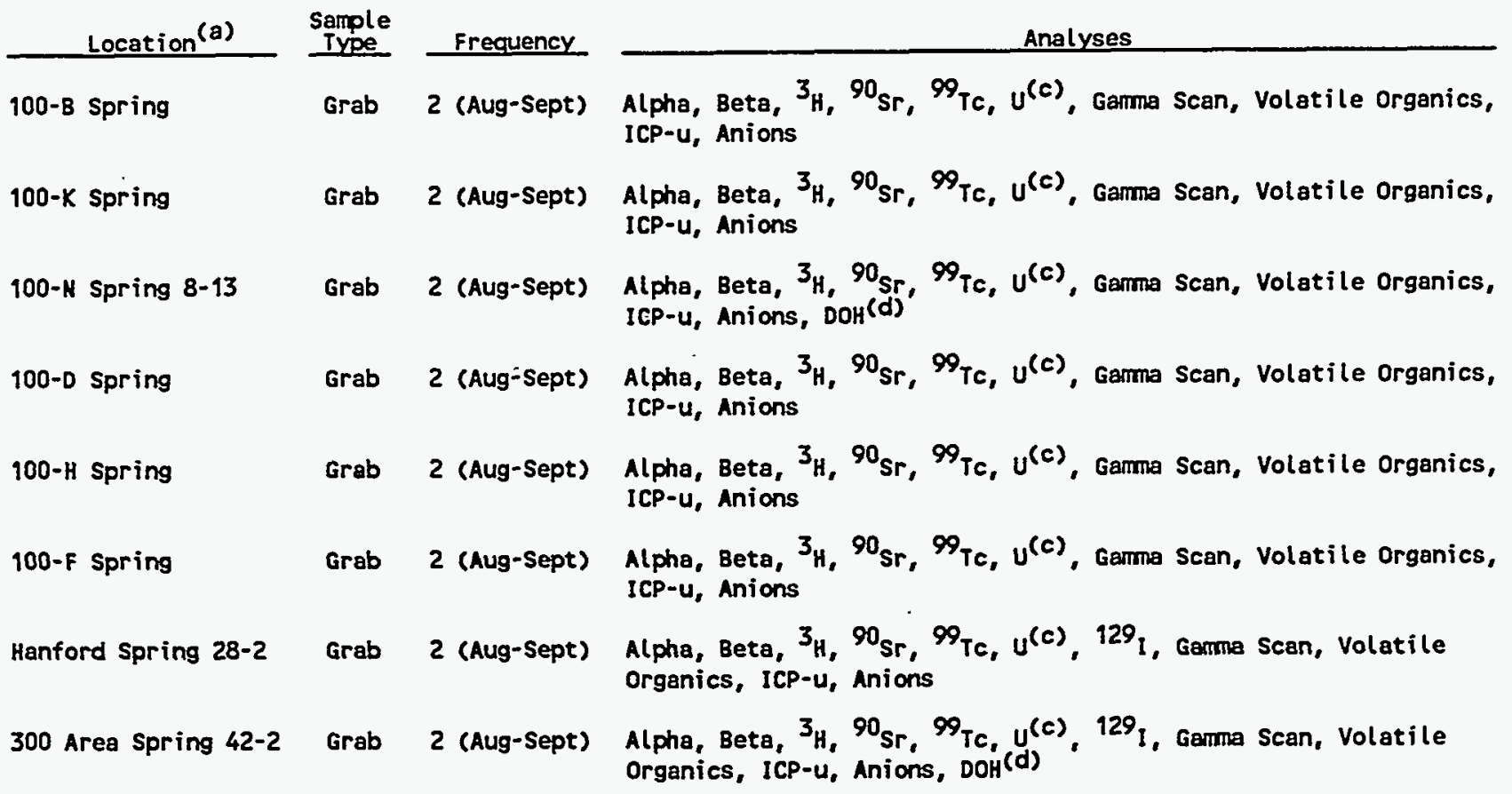

(a) Refer to Figure 2.1, Surface Water and Drinking Water Sampling Locations.

(b) Collected twice a year during low river flow season.

(c) Isotopic uranium.

(d) Duplicate sample with the Washington State Department of Health.

\subsection{ONSITE PONDS}

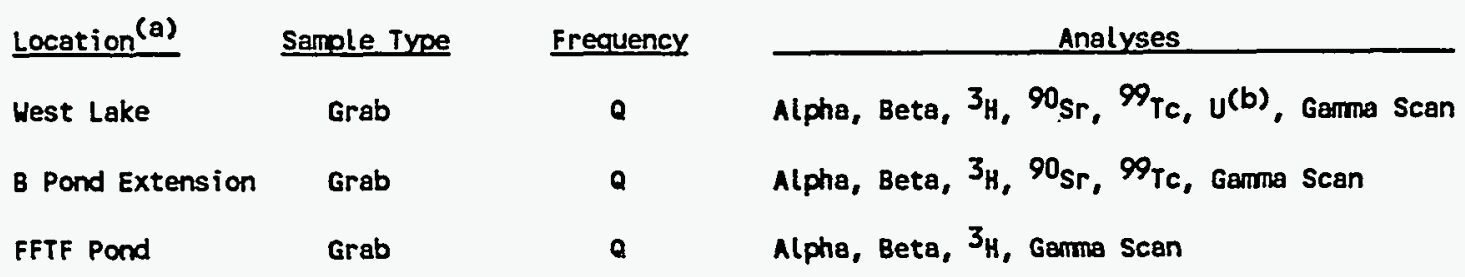

(a) Refer to Figure 2.1, Surface Water and Drinking Water Sampling Locations.

(b) Isotopic uranium. 


\subsection{OFFSITE WATER SYSTEMS}

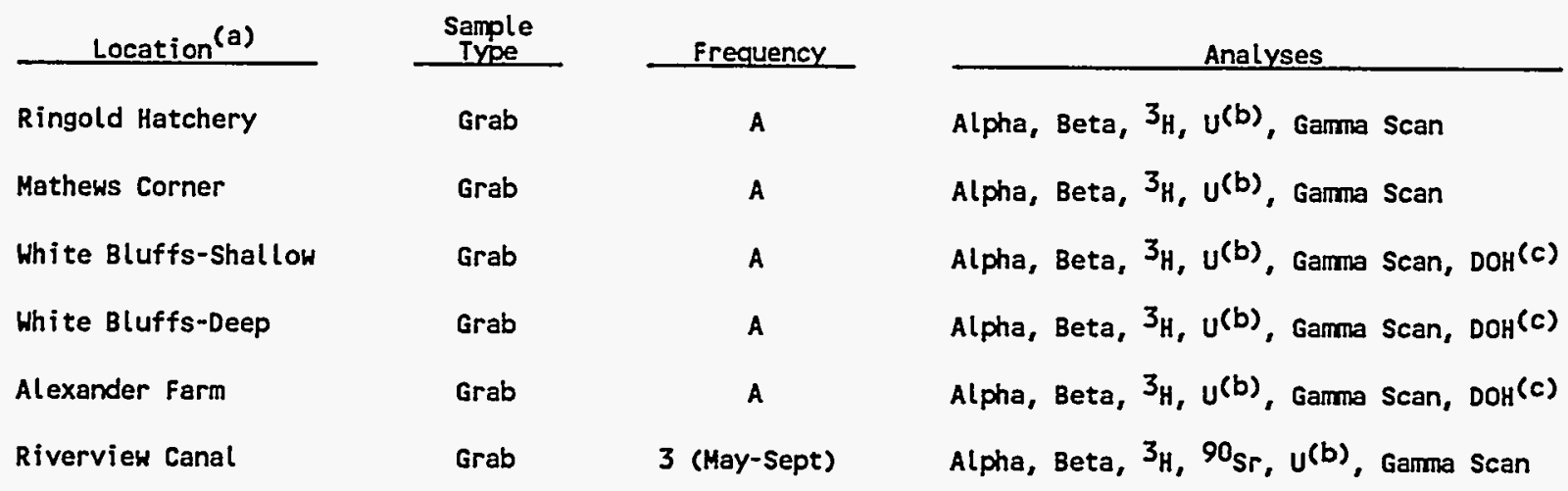

(a) Refer to Figure 2.1, Surface Water and Drinking Water Sampling Locations.

(c) Duplicate samples with the Washington State Department of Health.

\subsection{ONSITE DRINKING WATER}

Location (a)
100-B Area-River
100-D Area-River
Yakima Barricade

FFTF

300 Area

Firing Range

ALE Hdqtrs

$\begin{array}{cc}\begin{array}{c}\text { Sample } \\ \text { Type }\end{array} & \text { Frequency } \\ \text { Grab } & Q \\ \text { Grab } & 0 \\ \text { Grab } & 0 \\ \text { Grab } & \text { M } \\ & Q \\ & \text { A }\end{array}$

cumulative
Analyses

Alpha, Beta, Lo ${ }^{3} \mathrm{H},{ }^{90} \mathrm{Sr}$, Gamma Scan

Alpha, Beta, ${ }^{3}$, ${ }^{90} \mathrm{Sr}$, Gamma Scan, DoH(b)

Alpha, Beta, ${ }^{3}$, $90_{S r}$, Gamma Scan

$3_{\text {H }}$

Alpha, Beta, ${ }^{90} \mathrm{Sr}$, Gamma Scan, DoH $(\mathrm{b})$

Alpha, Beta, Lo ${ }^{3} \mathrm{H}, 9^{90} \mathrm{Sr},{ }^{99} \mathrm{TC}$, $u^{(c)}$, Gamma Scan

Alpha, Beta, $3_{\mathrm{H}}, 90 \mathrm{Sr}$, Gamma Scan

Alpha, Beta, $3_{\mathrm{H},} 90_{\mathrm{Sr}}$, Gamma Scan

(a) Refer to Figure 2.1 Surface Hater and Drinking Hater Sampling Locations.

(b) Duplicate sample collected 2nd quarter with Washington State Department of Health.

(c) Composite of monthly cumulative samples.

(d) Isotopic uranium. 


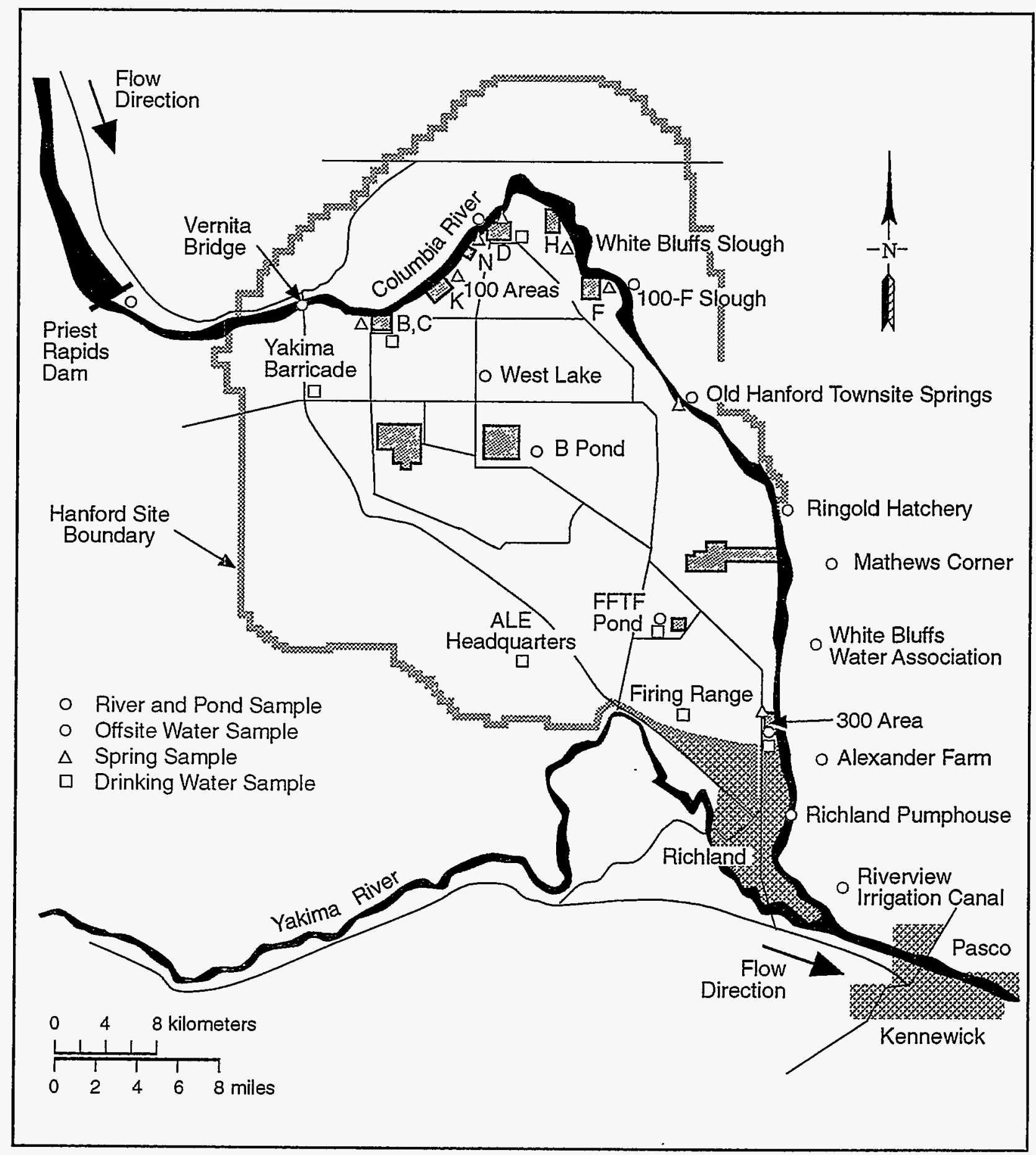

S9412049.9

Figure 2.1. Surface Water and Drinking Water Sampling Locations 


\subsection{FOODSTUFFS AND FARM PRODUCTS}

\subsection{WHOLE MILK}

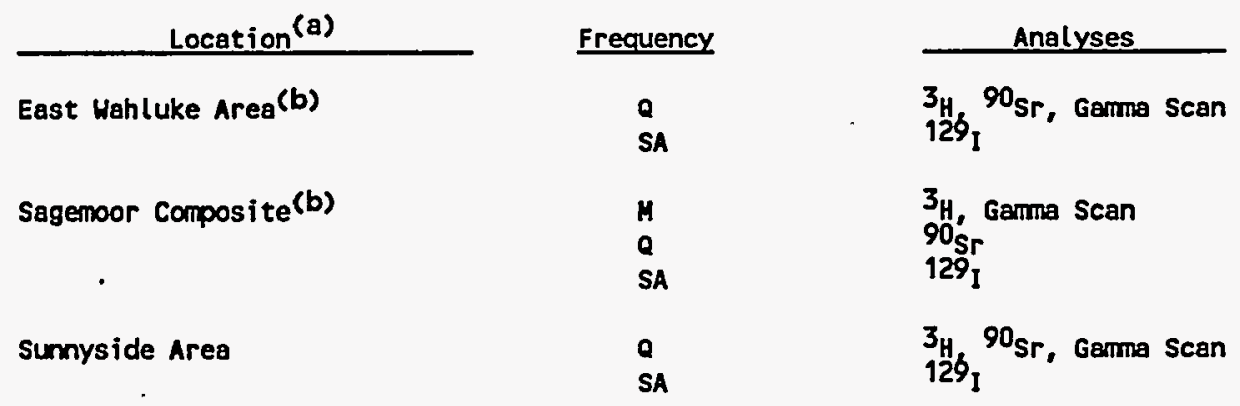

(a) Refer to Figure 3.1, Food and Farm Product Sampling Locations.

(b) Semple composited from three sources in each area.

\subsection{POULTRY AND EGGS}

\begin{tabular}{|c|c|c|c|}
\hline Location $^{(a)}$ & Sample Type & Erequency & Analyses \\
\hline Sagemoor Area & $\begin{array}{l}\text { Chicken } \\
\text { Eggs }\end{array}$ & $\hat{A}$ & $\begin{array}{l}90 \mathrm{Sr}, \text { Garma Scan, DOH } \\
{ }^{(b)} \\
90 \mathrm{Sr} \text {, Garma Scan, DOH }\end{array}$ \\
\hline Sunnyside Area & $\begin{array}{l}\text { Chicken } \\
\text { Eggs }\end{array}$ & $\begin{array}{l}A \\
A\end{array}$ & $\begin{array}{l}90 \mathrm{Sr} \text {, Gamma Scan, DOH }{ }^{(b)} \\
90 \mathrm{Sr} \text {, Gamma Scan, DOH }\end{array}$ \\
\hline
\end{tabular}

(a) Refer to Figure 3.1 Food and Farm Product Sampling Locations.

(b) Duplicate samples with the Hashington State Department of Heal th.

\subsection{BEEF}

Location(a)

Sagemoor Area

Riverview Area

Sunnyside Area
Frequency

A

A

$\mathbf{A}$
Analyses

${ }^{90} \mathrm{Sr}$, Garma Scan

$90 \mathrm{Sr}$, Gamma Scan, DOH(b)

$90_{\mathrm{Sr}}$, Garma Scan, DOH ${ }^{(b)}$

(a) Refer to Figure 3.1 Food and Farm Product Sampling Locations.

(b) Duplicate samples with the Washington State Department of Heaith. 


\subsection{LEAFY VEGETABLES}

Location $(a)$

East Wahluke Area

Sagemoor Area

Riverview Area

Surnyside Area
Frequency (b)

$B A^{(c)}$

A

A

A
Analyses

${ }^{90} \mathrm{Sr}$, Gamma Scan

$90_{\text {Sr, Gamma Scen, }} 129_{1-L E P}$

${ }^{90} \mathrm{Sr},{ }^{9} 9_{\mathrm{TC}}$, Gamma Scan, FDA $(d), \mathrm{DOH}^{(e)}$

90 Sr, Garma Scan, FDA ${ }^{(d)}, \mathrm{DOH}^{(e)}$

(a) Refer to Figure 3.1, food and Farm Product Sampling Locations.

(b) Three samples collected within each area.

(c) Biannual samples will next be collected in 1996.

(d) Duplicate samples sent to U.S. Food and Drug Administration.

(e) Dupl icate samples with the Hashington State Department of Health.

\subsection{VEGETABLES}

\begin{tabular}{|c|c|c|c|}
\hline Location $(a)$ & Sample Type & Frequency (b) & Analyses \\
\hline Rivervien Area & $\begin{array}{l}\text { Potatoes } \\
\text { Tomatoes } \\
\text { Carrots }\end{array}$ & $\begin{array}{l}\mathbf{A} \\
\mathbf{A} \\
\mathbf{A}\end{array}$ & 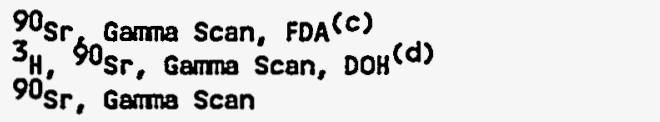 \\
\hline Horn Rapids Area & Potatoes & A & ${ }^{90} \mathrm{Sr},{ }^{99} \mathrm{TC}, \mathrm{Pu}(e), u^{(f)}$, Gamma Scan, $\mathrm{DOH}^{(d)}$ \\
\hline Sagemoor Area & Potatoes & A & ${ }^{90} \mathrm{Sr}, \mathrm{Pu}(\mathrm{e})$, Gamma Scan, FDA ${ }^{(c)}$ \\
\hline Sunnyside Area & Potatoes & $\boldsymbol{A}$ & ${ }^{90} \mathrm{Sr}, \mathrm{Pu}(e), u^{(f)},{ }^{99} \mathrm{Tc}$, Gamma Scan, FDA $(c)$ \\
\hline Harrah/Mapato Area & $\begin{array}{l}\text { Potatoes } \\
\text { Tomatoes }\end{array}$ & $\begin{array}{l}A \\
A\end{array}$ & 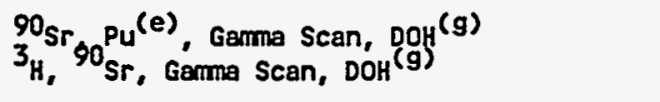 \\
\hline East Wahluke Area & Potatoes & $B A^{(h)}$ & $90 \mathrm{Sr}$, Gama Scan \\
\hline
\end{tabular}

(a) Refer to Figure 3.1, Food and Farm Product Sampling Locations.

(b) Three samples of each type collected within each area.

(c) Duplicate samples sent to U.S. Food and Drug Administration.

(d) Dupl icate samples with the Hashington State Department of Health.

(e) Isotopic plutonium.

(f) Isotopic uranium.

(9) Samples provided to PNL by Washington State Department of Health.

(h) Biannual samples will next be collected in 1996. 


\subsection{FRUIT}

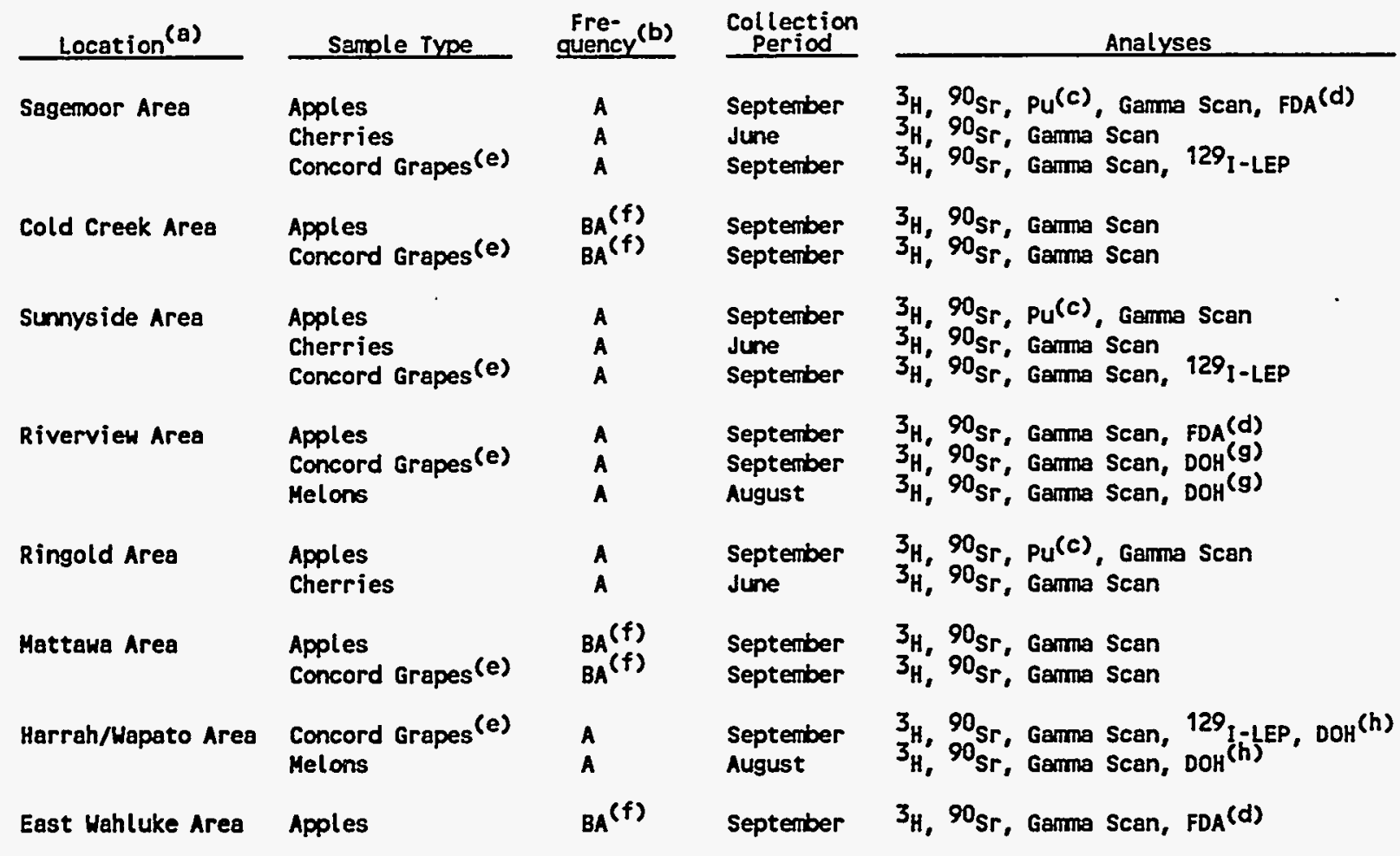

(a) Refer to Figure 3.1, Food and Farm Product Sampling Locations.

(b) Three samples of each type collected within each area.

(c) Isotopic plutonium.

(d) Duplicate samples sent to the U.S. Food and Drug Adninistration.

(e) Concord grapes preferred; table grapes acceptable if concord grapes are unavailable.

(f) Biannual samples will next be collected in 1996.

(g) Duplicate samples with the Washington State Department of Health.

(h) Samples provided to PHL by Washington state Department of Health.

\subsection{WINE}

\begin{tabular}{|c|c|c|c|c|}
\hline Location (a) & Sample Type & Erequency (b) & $\begin{array}{c}\text { Collection } \\
\text { Period } \\
\end{array}$ & Analyses \\
\hline Columbia Basin & $\begin{array}{l}\text { White } \\
\text { Red }\end{array}$ & $\hat{A}$ & $\begin{array}{l}\text { Decenber } \\
\text { Decenber }\end{array}$ & $\begin{array}{l}3 \mathrm{H}, \text { Gamma Scan, } \mathrm{DOH}^{(C)} \\
3_{\mathrm{H}, \text { Gamma Scan, DOH }}(\mathrm{C})\end{array}$ \\
\hline Yakima Valley & $\begin{array}{l}\text { White } \\
\text { Red }\end{array}$ & $\begin{array}{l}\mathbf{A} \\
\mathbf{A}\end{array}$ & $\begin{array}{l}\text { December } \\
\text { Decenber }\end{array}$ & $\begin{array}{l}3_{H} \text {, Gamma Scan, } \mathrm{DOH}^{(c)} \\
3_{H} \text {, Gamma Scan, DOH(c) }\end{array}$ \\
\hline
\end{tabular}

(a) Refer to Figure 3.1, Food and Farm Product Sampling Locations.

(b) Three samples of each type col lected within each area.

(c) Duplicate samples with the Hashington State Department of Health. 


\subsection{WHEAT AND ALFALFA}

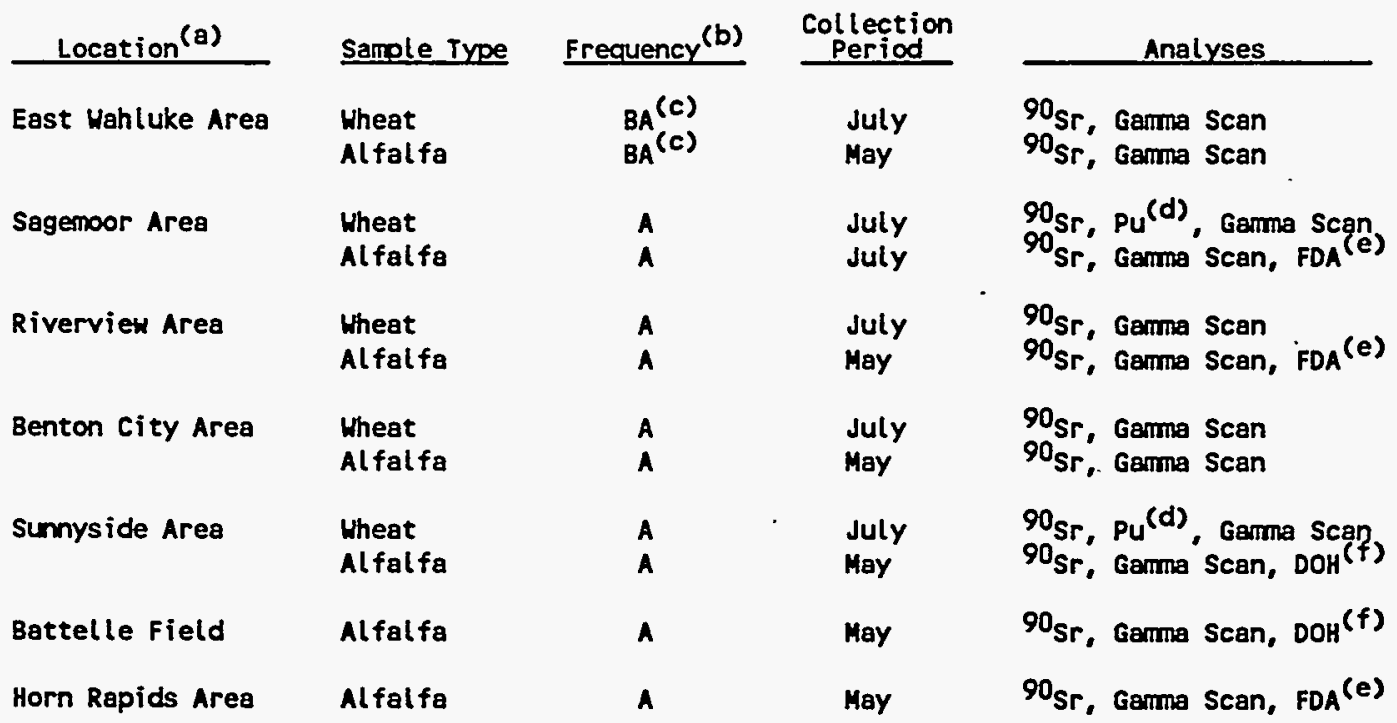

(a) Refer to Figure 3.1, Food and Farm Product Sampling Locations.

(b) Three samples of each type collected within each area.

(c) Biannual samples will next be collected in 1996.

(d) Isotopic plutonium.

(e) Duplicate samples seit to the U.S. Food and Drug Administration.

(f) Duplicate samples with the Washington State Department of Health. 


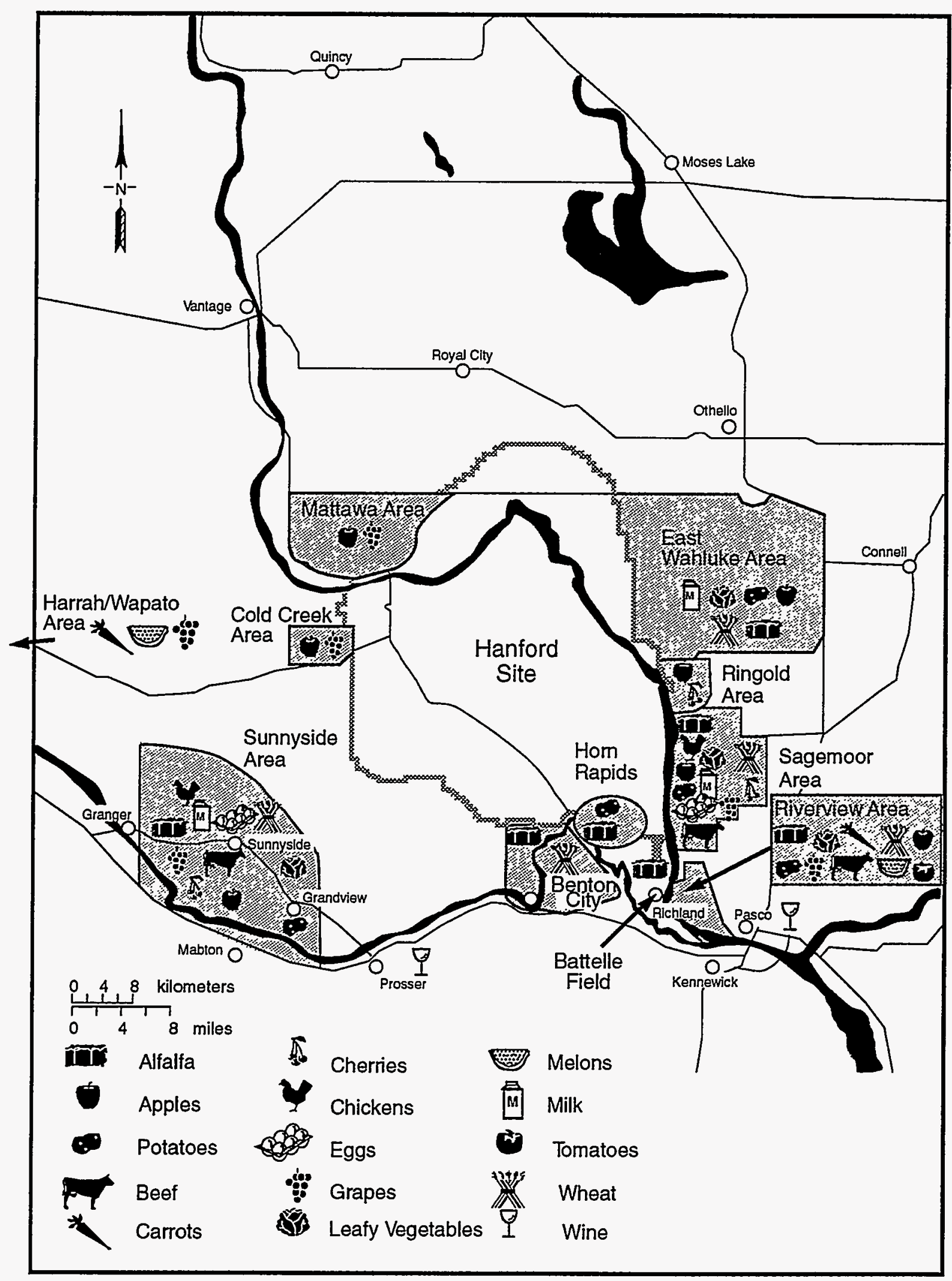

Figure 3.1. Food and Farm Product Sampling Locations

$\$ 9412049.5$ 


\subsection{WILDLIFE}

\subsection{AOUATIC BIOTA}

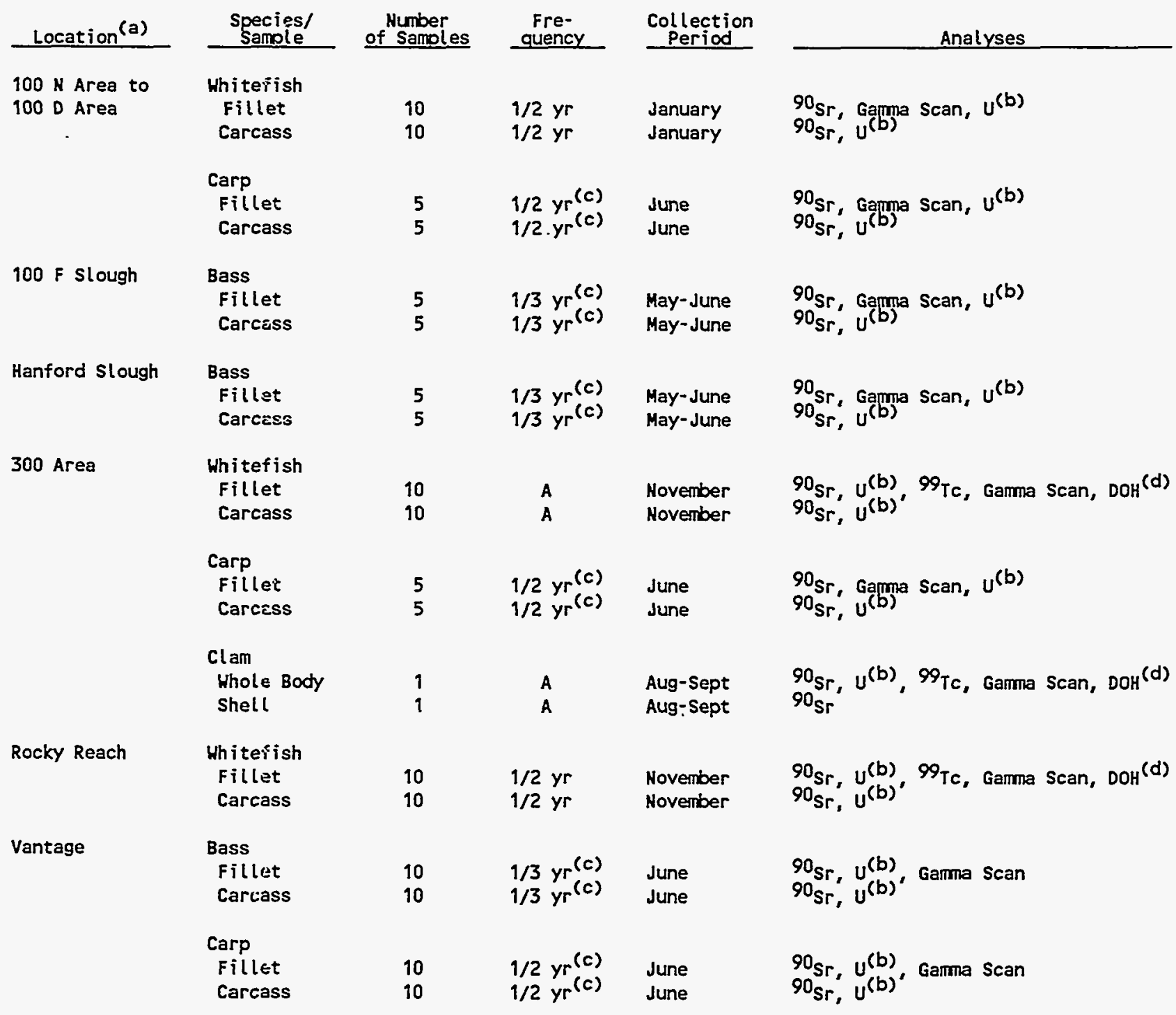

(a) Refer to Figure 4.1, Wildlife Sampling Areas.

(b) Isotopic uranium.

(c) Specified samples are collected once every 2 or 3 years with collection occurring in 1996 , unless otherwise statec (d) Dupl icate samples with the Washington State Department of Health. 


\subsection{GEESE}

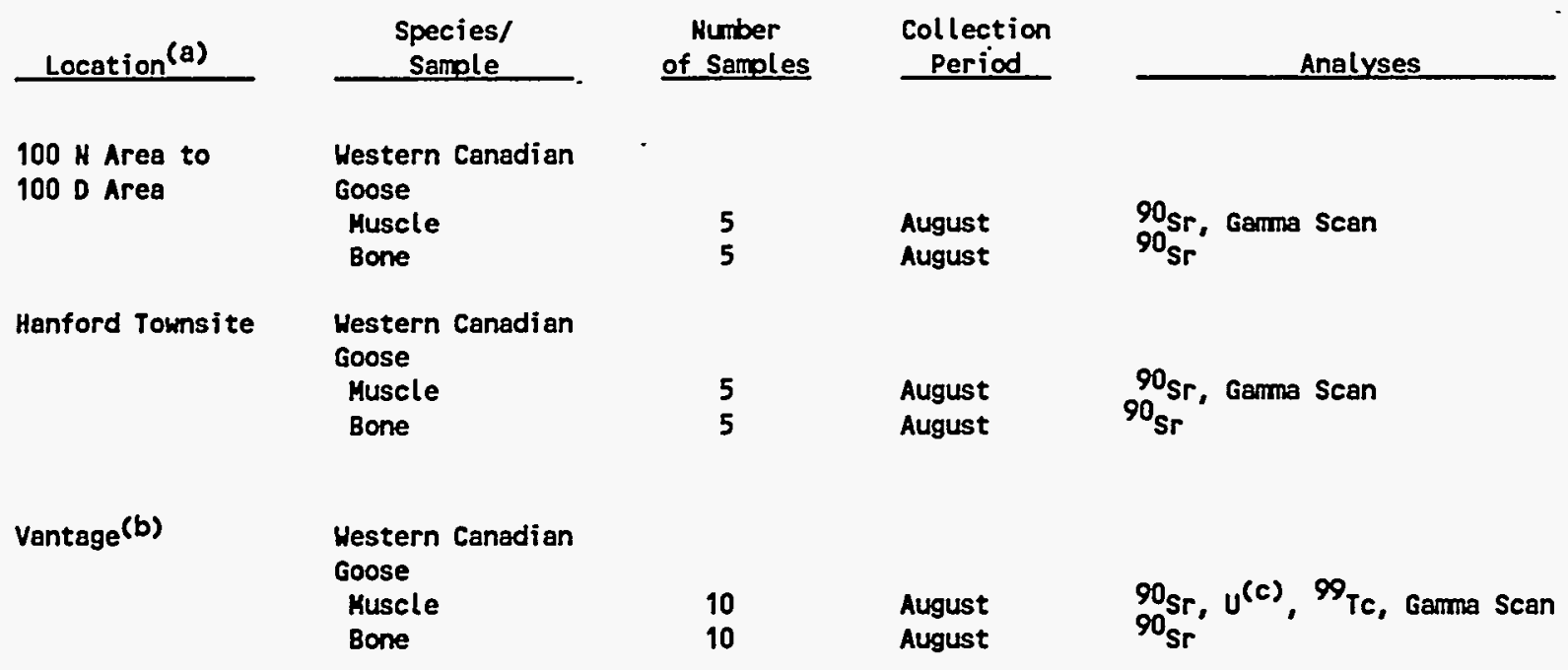

(a) Refer to Figure 4.1, Wildlife Sampling Areas.

(b) As avail lable.

(c) Isotopic uranium.

\subsection{GAME BIRDS}

\begin{tabular}{|c|c|c|c|c|}
\hline Location (a) & $\begin{array}{l}\text { Species/(b) } \\
\text { Sample }\end{array}$ & $\begin{array}{c}\text { Number } \\
\text { of Samples }\end{array}$ & $\begin{array}{c}\text { Collection } \\
\text { Period } \\
\end{array}$ & Analyses \\
\hline Near 100-N & $\begin{array}{l}\text { Pheasant } \\
\text { Muscle } \\
\text { Liver } \\
\text { Bone }\end{array}$ & $\begin{array}{l}3 \\
3 \\
3\end{array}$ & $\begin{array}{l}\text { January } \\
\text { January } \\
\text { January }\end{array}$ & $\begin{array}{l}9_{\mathrm{Sr}} \text {, Gamma Scan } \\
\mathrm{NRA} \text { (ć) } \\
90_{\mathrm{Sr}}\end{array}$ \\
\hline $\begin{array}{l}100 \mathrm{D} \text { Area to } \\
100 \mathrm{H} \text { Area }\end{array}$ & $\begin{array}{l}\text { Pheasant } \\
\text { Muscle } \\
\text { Liver } \\
\text { Bone }\end{array}$ & $\begin{array}{l}4 \\
4 \\
4\end{array}$ & $\begin{array}{l}\text { January } \\
\text { January } \\
\text { January }\end{array}$ & $\begin{array}{l}\text { Gamma Scan } \\
\operatorname{MRA}^{(C)} \\
90_{\mathrm{Sr}}\end{array}$ \\
\hline $\begin{array}{l}100 \text { H Area to } \\
100 \text { F Area }\end{array}$ & $\begin{array}{l}\text { Pheasant } \\
\text { Huscle } \\
\text { Liver } \\
\text { Bone }\end{array}$ & $\begin{array}{l}6 \\
6 \\
6\end{array}$ & $\begin{array}{l}\text { January } \\
\text { January } \\
\text { January }\end{array}$ & $\begin{array}{l}\text { Gemma Scan, } \mathrm{DOH}^{(\mathrm{d})} \\
\text { NRA (c) } \\
90_{\mathrm{Sr}}\end{array}$ \\
\hline Harrah/Hapato Area & $\begin{array}{l}\text { Pheasant } \\
\text { Huscle } \\
\text { Bone }\end{array}$ & $\begin{array}{l}1 \\
1\end{array}$ & $\begin{array}{l}\text { January } \\
\text { January }\end{array}$ & ${ }^{\text {Gomma }} \operatorname{Scan}_{\mathrm{Sr}, \mathrm{DOH}^{(e)}} \mathrm{DOH}^{(e)}$ \\
\hline Yakima County $(f)$ & $\begin{array}{l}\text { Pheasant } \\
\text { Huscle } \\
\text { Liver } \\
\text { Bone }\end{array}$ & $\begin{array}{l}10 \\
10 \\
10\end{array}$ & $\begin{array}{l}\text { September } \\
\text { September } \\
\text { September }\end{array}$ & $\begin{array}{l}90_{S r} u^{(g)} \text {, Gamma Scan } \\
{ }^{P u^{(h)}} \\
{ }^{90} \\
S r\end{array}$ \\
\hline
\end{tabular}

(a) Refer to Figure 4.1, Wildtife Sampl ing Areas.

(b) Pheasant preferred; chukar or quail acceptable if pheasant is unavailable.

(c) Not Routinely Analyzed (KRA) - Retain liver and bone to be analyzed for isotopic plutonium and $90 \mathrm{Sr}$, respectively, if $137 \mathrm{Cs}_{5}$ exceeds $100 \mathrm{pCi} / \mathrm{g}$ (wet weight) in muscle.

(d) Dupl icate sanples with the Washington State Department of Health.

(e) Samples provided to PHL by Washington State Department of Health.

(f) Background samples to be collected at least every 5 years and will next be collected in 1999.

(g) Isotopic uranium.

(h) Isotopic plutonium. 


\begin{tabular}{|c|c|c|c|c|}
\hline Location $(a)$ & $\begin{array}{l}\text { Species/ } \\
\text { Sample }\end{array}$ & $\begin{array}{c}\text { Number } \\
\text { of Samiles }\end{array}$ & $\begin{array}{l}\text { Collection } \\
\text { Period } \\
\end{array}$ & Analyses \\
\hline 100 N Area & $\begin{array}{l}\text { Mule } \\
\text { Muscle } \\
\text { Liver } \\
\text { Bone }\end{array}$ & $\begin{array}{l}2 \\
2 \\
2\end{array}$ & $\begin{array}{l}\text { December } \\
\text { December } \\
\text { December }\end{array}$ & $\begin{array}{l}\text { Gamma Scan } \\
\mathrm{Pu}^{(b)} \\
{ }^{\circ} \mathrm{Sr}\end{array}$ \\
\hline 200 Ponds & $\begin{array}{l}\text { Mule } \\
\text { Muscle } \\
\text { Liver } \\
\text { Bone }\end{array}$ & $\begin{array}{l}2 \\
2 \\
2\end{array}$ & $\begin{array}{l}\text { December } \\
\text { December } \\
\text { December }\end{array}$ & $\begin{array}{l}\text { Gamma Scan } \\
\mathrm{Pu}(\mathrm{b}) \\
9_{\mathrm{Sr}}\end{array}$ \\
\hline $\begin{array}{l}\text { Road Kill at } \\
\text { Onsite Locations(c) }\end{array}$ & $\begin{array}{l}\text { Mule } \\
\text { Muscle } \\
\text { Liver }\end{array}$ & $\begin{array}{l}6 \\
6\end{array}$ & $\begin{array}{l}\text { Annual } \\
\text { Annual }\end{array}$ & $\begin{array}{l}\text { Gamma Scan } \\
\mathrm{Pu}^{(\mathrm{b})}\end{array}$ \\
\hline Background(c), & $\begin{array}{l}\text { Mule } \\
\text { Muscle } \\
\text { Liver } \\
\text { Bone }\end{array}$ & $\begin{array}{l}2^{(d)} \\
2^{(d)} \\
2^{(d)}\end{array}$ & $\begin{array}{l}\text { October } \\
\text { october } \\
\text { October }\end{array}$ & $\begin{array}{l}\text { Gamma Scan } \\
\mathrm{Pu}(\mathrm{b}) \\
{ }^{9} \mathrm{Sr}_{\mathrm{r}}\end{array}$ \\
\hline
\end{tabular}

(a) Refer to Figure 4.1, Wildifife Sampling Areas.

(b) Isotopic plutonium.

(c) As avai lable, according to location.

(d) One west side deer will be provided to PNL by Washington State Department of Health.

\subsection{RABBITS}

\begin{tabular}{|c|c|c|c|c|}
\hline Location (a) & $\begin{array}{r}\text { Type/ } \\
\text { Semple } \\
\end{array}$ & $\begin{array}{c}\text { Number } \\
\text { of Samples }\end{array}$ & $\begin{array}{l}\text { Collection } \\
\text { Period } \\
\end{array}$ & Analyses \\
\hline 100 N Area & $\begin{array}{l}\text { Cottontail } \\
\text { Muscle } \\
\text { Liver } \\
\text { Bone }\end{array}$ & $\begin{array}{l}4 \\
4 \\
4\end{array}$ & $\begin{array}{l}\text { April } \\
\text { April } \\
\text { April }\end{array}$ & $\begin{array}{l}\text { Gamma Scan, } \mathrm{DOH}^{(b)} \\
\mathrm{Pu}^{(\mathrm{c})} \\
{ }^{\mathrm{P}_{\mathrm{Sr}}}\end{array}$ \\
\hline 200 E Area & $\begin{array}{l}\text { Jack Rabbit } \\
\text { Muscle } \\
\text { Liver } \\
\text { Bone }\end{array}$ & $\begin{array}{l}4 \\
4 \\
4\end{array}$ & $\begin{array}{l}\text { April } \\
\text { April } \\
\text { April }\end{array}$ & $\begin{array}{l}\text { Gamma Scan } \\
\mathrm{Pu}(\mathrm{c}) \\
90_{\mathrm{Sr}}\end{array}$ \\
\hline 200 West & $\begin{array}{l}\text { Jack Rabbit } \\
\text { Muscle } \\
\text { Liver } \\
\text { Bone }\end{array}$ & $\begin{array}{l}4 \\
4 \\
4\end{array}$ & $\begin{array}{l}\text { April } \\
\text { April } \\
\text { April }\end{array}$ & $\begin{array}{l}\text { Gamma Scan } \\
\mathrm{Pu}^{(c)} \\
9_{\mathrm{Sr}}\end{array}$ \\
\hline Morth 300 Area & $\begin{array}{l}\text { Cottontail } \\
\text { Muscle } \\
\text { Liver } \\
\text { Bone }\end{array}$ & $\begin{array}{l}4 \\
4 \\
4\end{array}$ & $\begin{array}{l}\text { April } \\
\text { April } \\
\text { Aprit }\end{array}$ & $\begin{array}{l}\text { Garma Scan } \\
\mathrm{Pu}^{(c)} \\
{ }^{\circ}{ }_{\mathrm{Sr}}\end{array}$ \\
\hline
\end{tabular}




\subsection{RABBITS (contd)}

\begin{tabular}{|c|c|c|c|c|}
\hline Location (a) & $\begin{array}{l}\text { Type/ } \\
\text { Sample }\end{array}$ & $\begin{array}{c}\text { Number } \\
\text { of Samples }\end{array}$ & $\begin{array}{l}\text { Collection } \\
\text { Period } \\
\end{array}$ & Analyses \\
\hline Boardman, Orégon(d) & $\begin{array}{l}\text { Jack Rabbit } \\
\text { Kuscle } \\
\text { Liver } \\
\text { Bone }\end{array}$ & $\begin{array}{l}10 \\
10 \\
10\end{array}$ & $\begin{array}{l}\text { April } \\
\text { April } \\
\text { April }\end{array}$ & $\begin{array}{l}\text { Gamma Scan, } \mathrm{DOH}^{(b)} \\
\mathrm{Pu}^{(\mathrm{c})} \\
\mathrm{OO}_{\mathrm{Sr}}\end{array}$ \\
\hline Boarcman, Oregon(d) & $\begin{array}{l}\text { Cottontail } \\
\text { Kuscle } \\
\text { Liver } \\
\text { Bone }\end{array}$ & $\begin{array}{l}10 \\
10 \\
10\end{array}$ & $\begin{array}{l}\text { April } \\
\text { April } \\
\text { April }\end{array}$ & $\begin{array}{l}\text { Gamma Scan, } \mathrm{DOH}^{(\mathrm{b})} \\
\mathrm{Pu}^{(\mathrm{c})} \\
{ }^{90_{\mathrm{Sr}}}\end{array}$ \\
\hline
\end{tabular}

(a) Refer to Figure 4.1 Hild ife Sampling Areas.

(b) Dupl icate samples with the Hashington State Department of Health.

(d) Background samples to be collected at least every 5 years and will next be collected in 1995. 


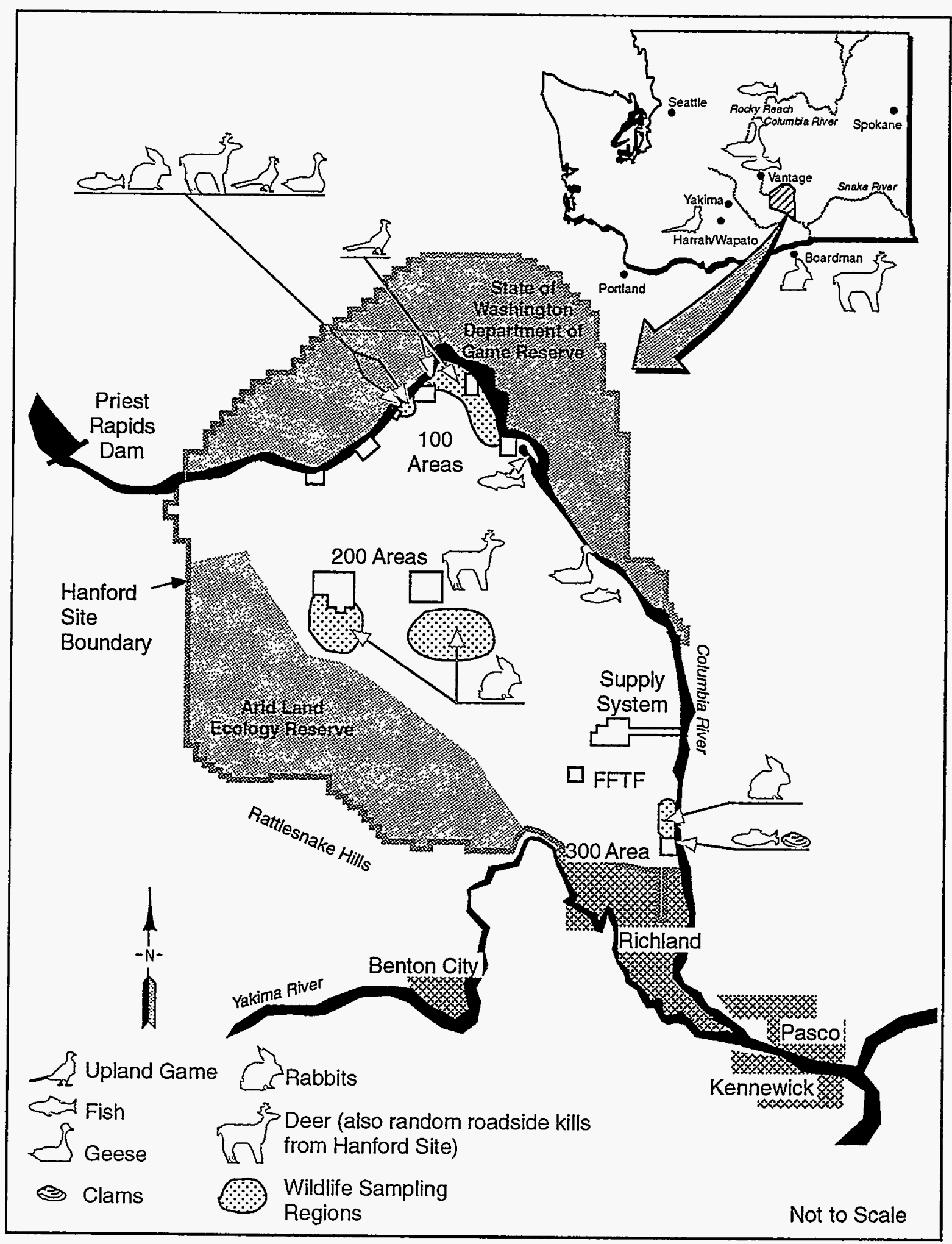

Figure 4.1. Wildlife Sampling Areas 


\subsection{SOIL AND VEGETATION}

\subsection{SOIL}

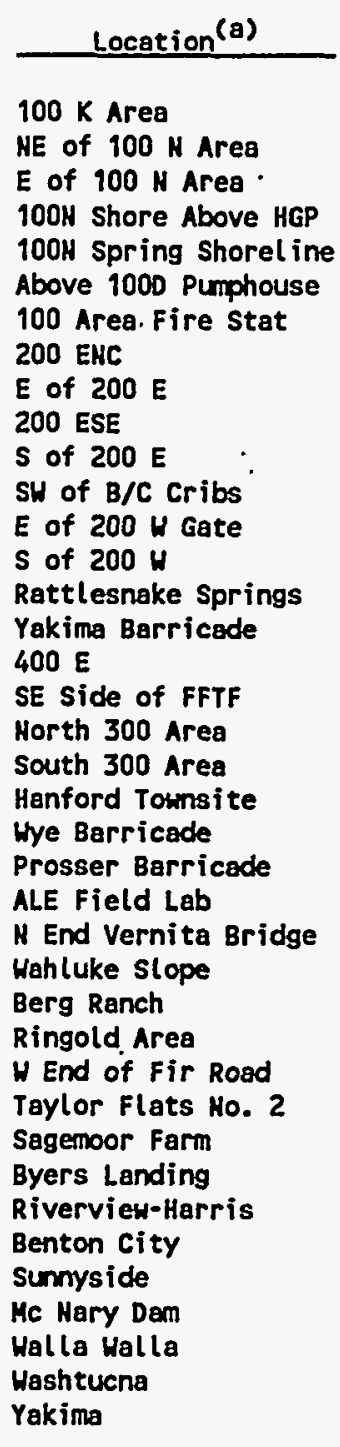

\begin{tabular}{|c|c|}
\hline $\begin{array}{c}\text { Fre- } \\
\text { quency }\end{array}$ & $\begin{array}{l}\text { Collection } \\
\text { Period }\end{array}$ \\
\hline A & July \\
\hline A & July \\
\hline A & July \\
\hline A & July \\
\hline A & July \\
\hline A & July \\
\hline A & July \\
\hline A & July \\
\hline A & July \\
\hline A & suly \\
\hline A & July \\
\hline A & July \\
\hline$A$ & July \\
\hline A (e) & July \\
\hline$q / 3 y r^{(e)}$ & July \\
\hline $1 / 3 y_{r}^{(e)}$ & July \\
\hline$A$ & July \\
\hline$A$ & July \\
\hline A & July \\
\hline A & July \\
\hline $1 / 3 y r_{(e)}^{(e)}$ & July \\
\hline $1 / 3 y r r^{(e)}$ & July \\
\hline $1 / 3 y r r^{(e)}$ & July \\
\hline $1 / 3 y r r^{(e)}$ & July \\
\hline $1 / 3$ yr (e) & July \\
\hline $1 / 3$ yr (e) & July \\
\hline $1 / 3 y r^{(e)}$ & July \\
\hline$\hat{A}_{y r}^{A}(e)$ & July \\
\hline $\begin{array}{l}1 / 3 \text { yr } \\
1 / 3 \text { e }\end{array}$ & July \\
\hline A & July \\
\hline A & July \\
\hline$A^{A}(e)$ & July \\
\hline $1 / 3 y r^{(e)}$ & July \\
\hline $1 / 5^{A} y r^{(e)}$ & $\begin{array}{l}\text { July } \\
\text { July }\end{array}$ \\
\hline $1 / 5 y^{(e)}$ & July \\
\hline${ }_{A}^{1 / 5} y^{(e)}$ & $\begin{array}{l}\text { July } \\
\text { July }\end{array}$ \\
\hline
\end{tabular}

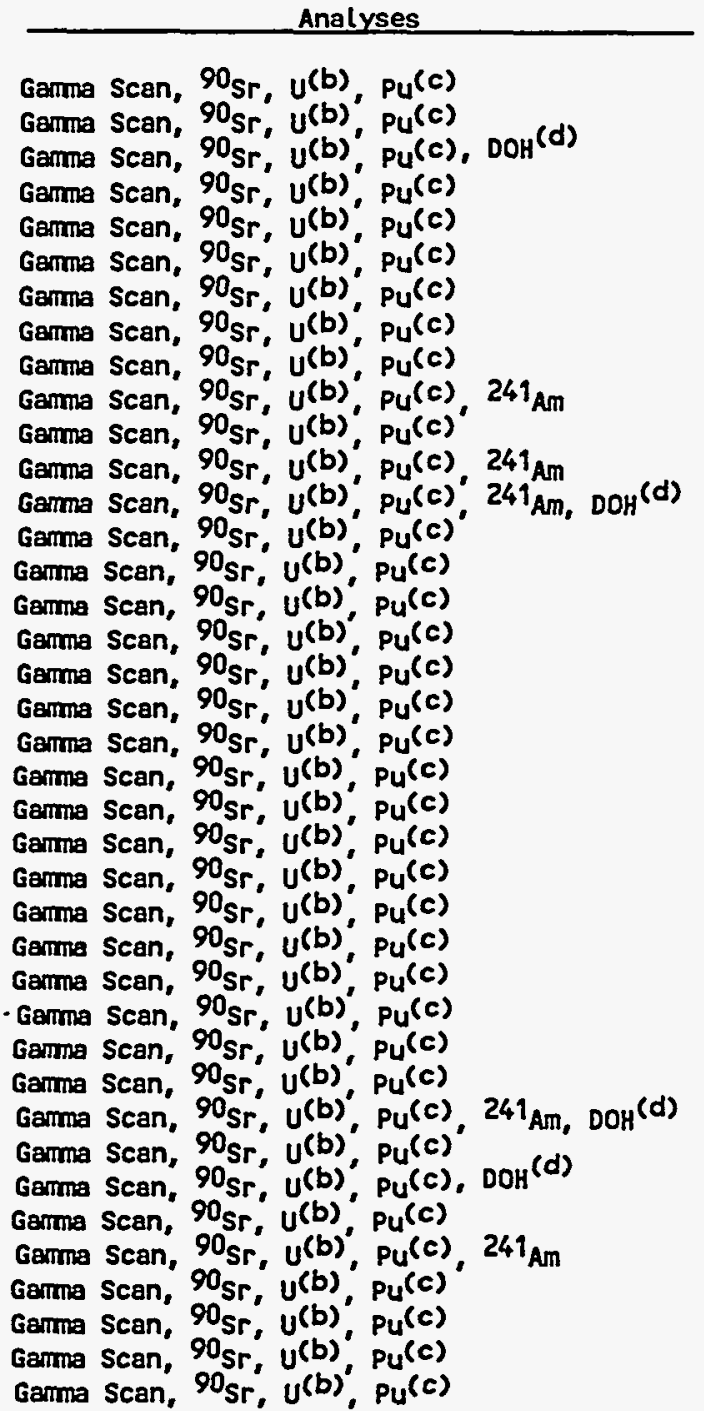

(a) Beffer to Figure 5.1, Soil and Vegetation Sempling Locations.

(b) $235 U$-LEP, 238U-LEP.

(c) Isotopic plutonium.

(d) Dupl icate samples with the Hashington state Department of Health

(e) Specified samples are collected once every 3 or 5 years, respectively. The next samples for 1/3-year and 1/5-year locations will be 1996 and 1995, respectively. 


\subsection{VEGETATION}

Location $^{(a)}$

$100 \times$ Area

NE of 100 N Area

$E$ of $100 \mathrm{~N}$ Area

100N Spring Shoreline

E of $200 \mathrm{~W}$ Gate

300 Area Shoreline

Hanford Tounsite

Hanfrd Twnsite HRM28

Ringold

Sagernoor Farm

Byers Landing

Riverview-Harris

Sunnyside

Yakima

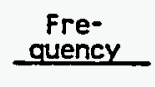

A

A

$\begin{array}{cc}\hat{A} \hat{A r}_{\text {(d) }} & \text { July } \\ \hat{A} & \text { July } \\ \hat{A} & \text { July } \\ \hat{A} & \text { July } \\ \hat{A} & \text { July } \\ \hat{A} & \text { July } \\ \hat{A} & \text { July } \\ \hat{A} & \text { July } \\ & \text { July } \\ & \text { July }\end{array}$

Analyses

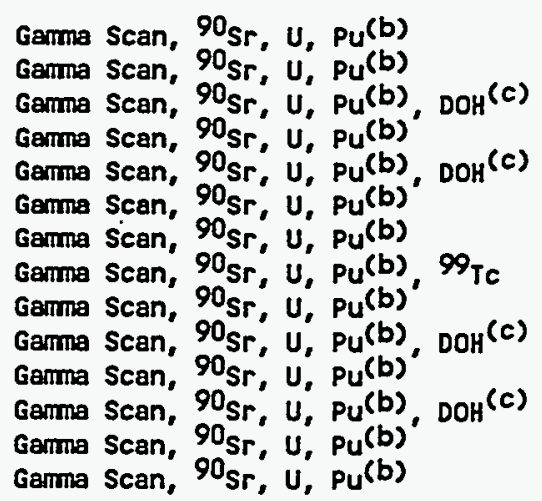

(a) Refer to Figure 5.1, Soil and Vegetation Sempling Locations.

(b) Isotopic plutonium.

(c) Duplicate samples with the Washington State Department of Vildlife.

(d) Specified samples are collected once every 3 years and will next be collected in 1996. 


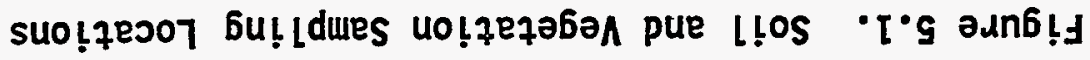

\section{ZLtTES}

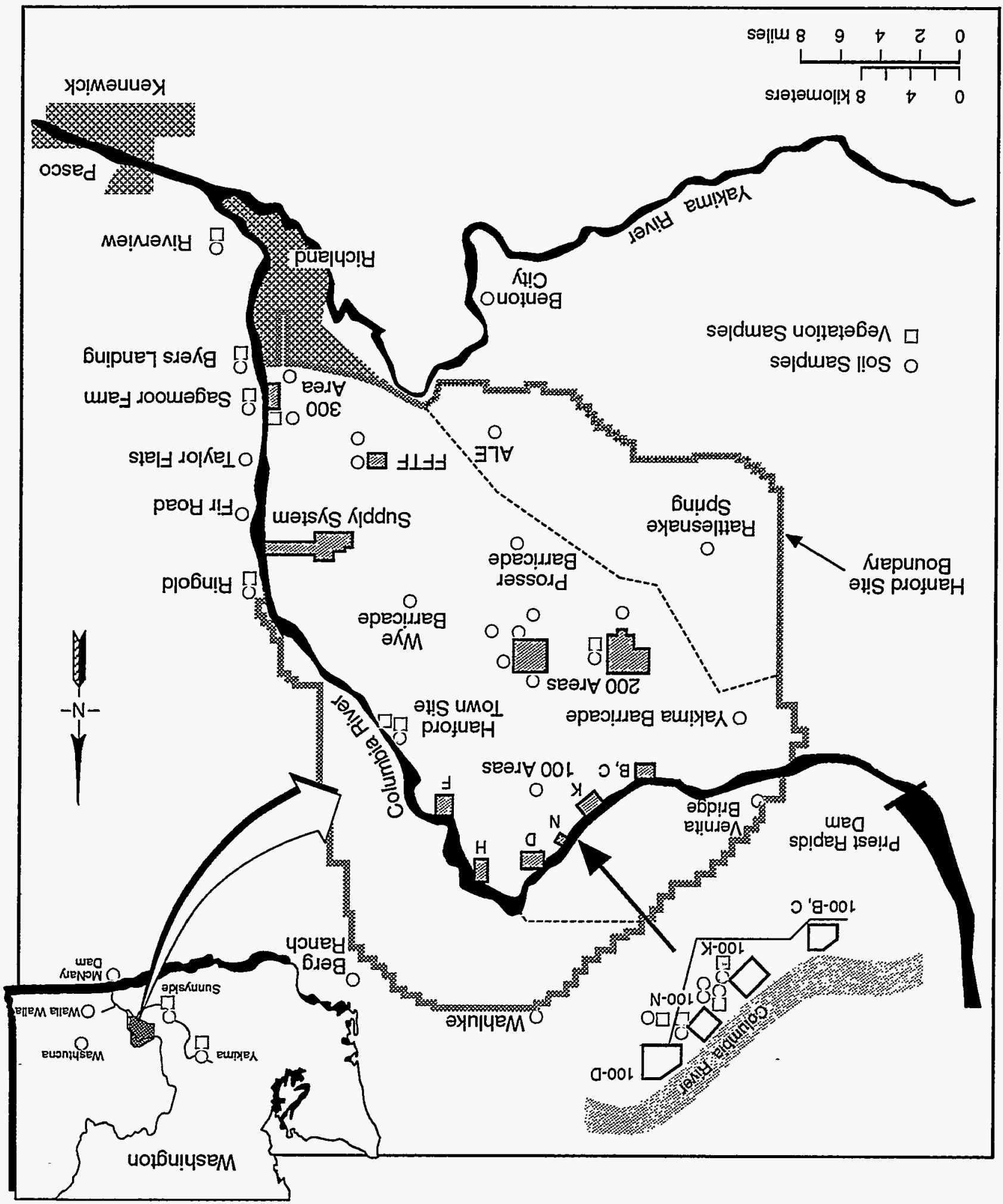




\subsection{SEDIMENT}

Location (a)

River

Mc Nary Dam

McNary Oregon Shore

MeNary-1/3 OR. Shore

McNary-2/3 OR. Shore

McNary-Wash. Shore

Priest Rapids Dan

PRD-Grant County

PRD-1/3 Grant Shore

PRD-2/3 Grant Shore

PRD-Yakima Cnty Shor

White Bluffs Slough

$100 \mathrm{~F}$ slough

Hanford Slough

Richland

\section{Springs}

100-B Spring

100 N Spring 8-13

Hanford Spring 28-2

300 Area Spring 42-2
Frequency

Gamma Scan, ${ }^{90} \mathrm{Sr}, \mathrm{u}^{(b)}, \mathrm{Pu}(\mathrm{c}), I C P-\mathrm{u}, \mathrm{DOH}(\mathrm{d})$

Gamma Scan, $90_{\mathrm{Sr}}, \mathrm{u}(\mathrm{b}), \mathrm{Pu}(\mathrm{c}), \mathrm{ICP}-\mathrm{u}, \mathrm{DOH}(\mathrm{d})$

Garma Scan, $90 \mathrm{Sr}, \mathrm{u}^{(b)}, \mathrm{Pu}(\mathrm{c}), \mathrm{ICP}-\mathrm{u}, \mathrm{DOH}{ }^{(d)}$

Gamma Scan, ${ }^{90} \mathrm{Sr}, \mathrm{U}^{(b)}, \mathrm{Pu}(c), I C P-u, \mathrm{DOH}^{(d)}$

Gamma Scan, ${ }^{90} \mathrm{Sr}, \mathrm{U}^{(b)}, \mathrm{Pu}(\mathrm{c}), I C P-\mathrm{u}, \mathrm{DOH}(\mathrm{d})$

Gamma Scan, $90 \mathrm{Sr}, u^{(b)}, \mathrm{Pu}^{(c)}, \mathrm{ICP}-\mathrm{u}, \mathrm{DOH}(\mathrm{d})$

Gamma Scan, ${ }^{90} \mathrm{Sr}, \mathrm{u}(b), P \mathrm{u}^{(c)}, I C P-u, D O H(d)$

Gamm Scan, ${ }^{90} \mathrm{Sr}, \mathrm{u}(b), \mathrm{Pu}^{(c)}, \mathrm{ICP}-\mathrm{u}, \mathrm{DOH}(\mathrm{d})$

Gamma Scan, ${ }^{90} \mathrm{Sr}, \mathrm{u}^{(b)}, \mathrm{Pu}(\mathrm{c}), \mathrm{ICP}-\mathrm{u}$

Gamma Scan, ${ }^{90} \mathrm{Sr}, \mathrm{u}^{(b)}, \mathrm{Pu}(\mathrm{c}), \mathrm{ICP}-\mathrm{u}$

Gamma Scan, ${ }^{90} \mathrm{Sr}, \mathrm{u}^{(b)}, \mathrm{Pu}(\mathrm{c}), \mathrm{ICP}-\mathrm{u}$

Gamma Scan, ${ }^{90} \mathrm{Sr}, \mathrm{u}^{(b)}, \mathrm{Pu}(\mathrm{c}), \mathrm{ICP}-\mathrm{u}$

A Gamma scan, ${ }^{90} \mathrm{Sr}, \mathrm{u}^{(\mathrm{b})}, \mathrm{ICP}-\mathrm{u}$

A Garma Scan, $90 \mathrm{Sr}, \mathrm{u}^{(b)}, \mathrm{ICP}-\mathrm{u}$

A Gamma Scan, ${ }^{90} \mathrm{Sr}, \mathrm{u}^{(\mathrm{b})}, \mathrm{ICP}-\mathrm{u}$

A

Gamma Scan, ${ }^{90} \mathrm{Sr}, \mathrm{u}^{(\mathrm{b})}, \mathrm{ICP}-\mathrm{u}$

(a) Refer to Figure 6.1, Sediment Sampling Locations.

(b) $235 \mathrm{U}$-LEP, $238 \mathrm{U}-\mathrm{LEP}$.

(c) Isotopic plutonium

(d) Duplicate samples with the Hashington State Department of Health. 


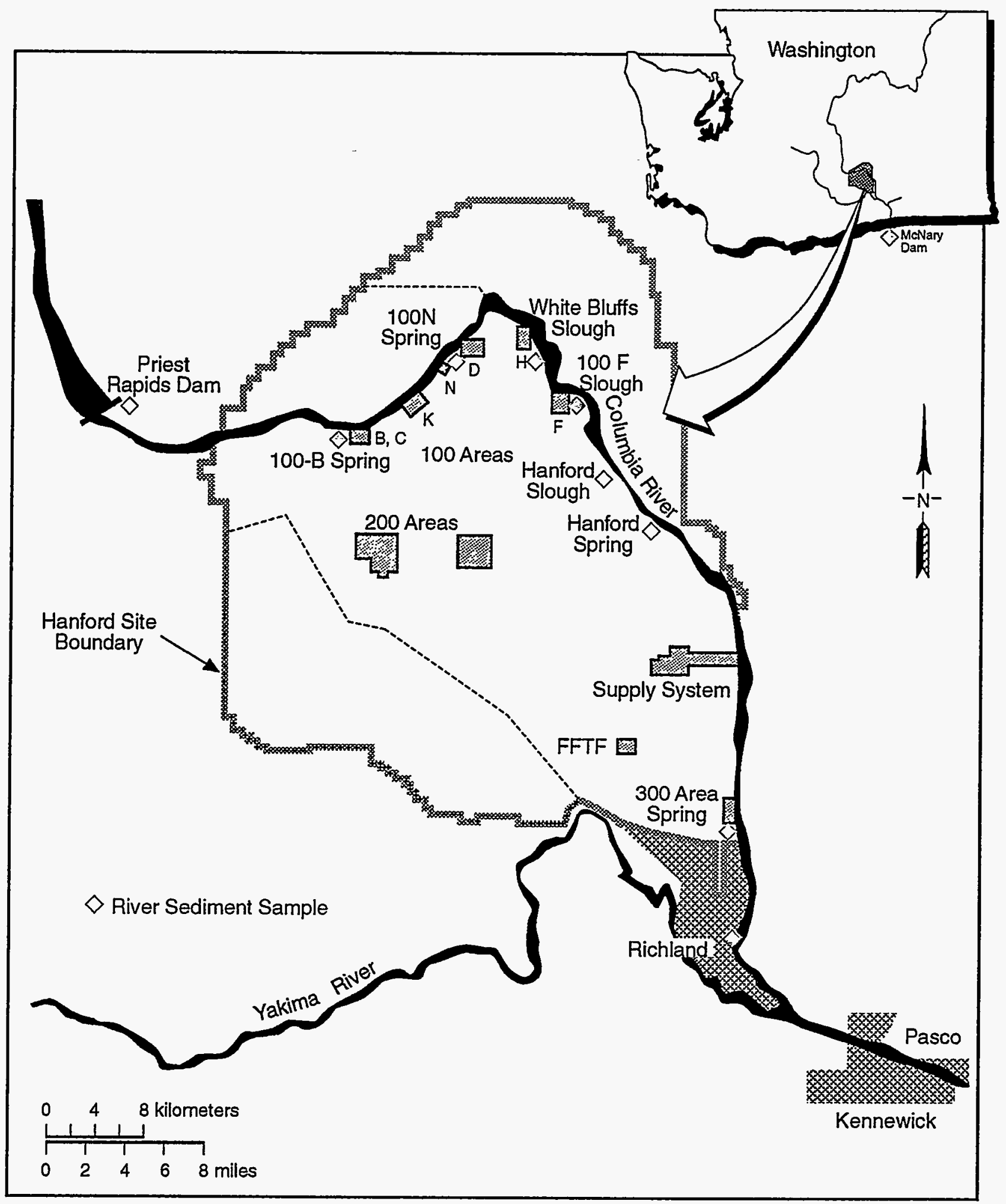

S9412049.7

Figure 6.1. Sediment Sampling Locations 


\subsection{EXTERNAL RADIATION}

\subsection{THERMOLUMINESCENT DOSIMETERS (TLDS)}

\subsubsection{Terrestria? Locations}

\begin{tabular}{|c|c|c|c|c|}
\hline Location & $\begin{array}{l}\text { Location } \\
\text { Number }\end{array}$ & Frequency & Measurement & Instrument \\
\hline \multicolumn{5}{|l|}{ Onsite ${ }^{(a)}$} \\
\hline 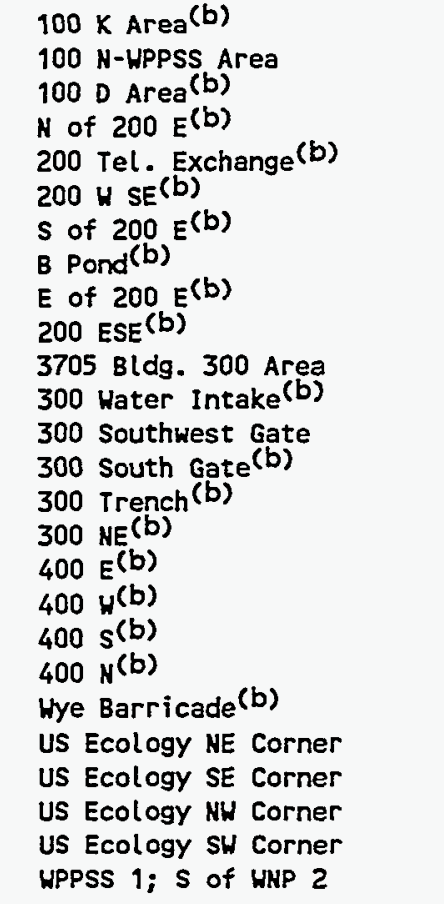 & $\begin{array}{r}1 \\
2 \\
3 \\
4 \\
5 \\
6 \\
7 \\
8 \\
9 \\
10 \\
11 \\
12 \\
13 \\
14 \\
15 \\
16 \\
17 \\
18 \\
19 \\
20 \\
21 \\
22 \\
23 \\
24 \\
25 \\
26\end{array}$ & 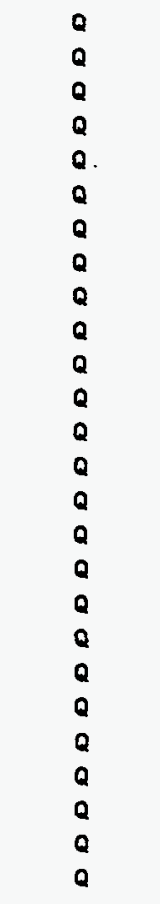 & 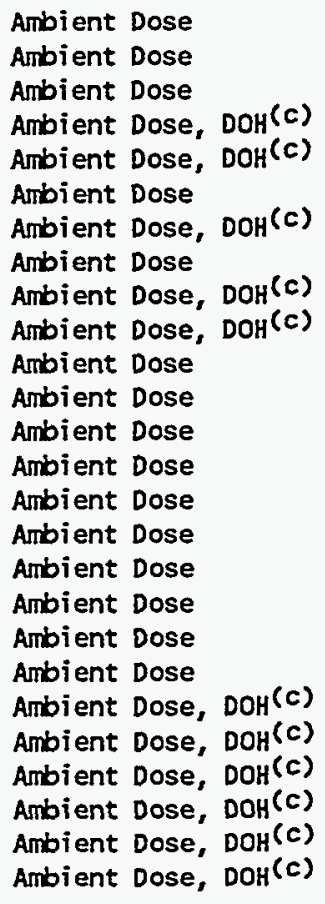 & \\
\hline \multicolumn{5}{|l|}{ Perimeter (d) } \\
\hline $\begin{array}{l}\text { Ringold Met Tower(b) } \\
\text { Byers Landing(b) } \\
\text { Battelle Complex(b) } \\
\text { WPPSS 4; WPS Warehse } \\
\text { WPPSS 8; Fir Road }\end{array}$ & $\begin{array}{l}1 \\
2 \\
3 \\
4 \\
5\end{array}$ & $\begin{array}{l}Q \\
Q \\
Q \\
Q \\
Q\end{array}$ & $\begin{array}{l}\text { Ambient Dose } \\
\text { Ambient Dose } \\
\text { Ambient Dose } \\
\text { Ambient Dose, DOH(c) } \\
\text { Ambient Dose, DOH(c) }\end{array}$ & \\
\hline \multicolumn{5}{|l|}{ Community(d) } \\
\hline $\begin{array}{l}\text { Basin City School }(b)(e) \\
\text { Pasco(b)(e) } \\
\text { Kennewick-Ely Street(b)(e) } \\
\text { Benton City (b) } \\
\text { Mattawa(b) } \\
\text { Othello(b) } \\
\text { Edwin Markham School }(b)(e) \\
\text { Leslie Groves Rchlnd }(b)(e)\end{array}$ & $\begin{array}{r}6 \\
7 \\
8 \\
9 \\
10 \\
11 \\
12 \\
13\end{array}$ & $\begin{array}{l}0 \\
0 \\
0 \\
0 \\
0 \\
0 \\
0 \\
0\end{array}$ & $\begin{array}{l}\text { Ambient Dose } \\
\text { Ambient Dose } \\
\text { Ambient Dose } \\
\text { Ambient Dose } \\
\text { Ambient Dose } \\
\text { Ambient Dose } \\
\text { Ambient Dose } \\
\text { Ambient Dose }\end{array}$ & $\begin{array}{l}\text { PIC } \\
\text { PIC }\end{array}$ \\
\hline
\end{tabular}




\subsubsection{Terrestrial Locations (contd)}

\begin{tabular}{|c|c|c|c|c|}
\hline Locatio & $\begin{array}{l}\text { Location } \\
\text { Number }\end{array}$ & Frequency & Measurement & Instrument \\
\hline \multicolumn{5}{|l|}{ Distant ${ }^{(d)}$} \\
\hline $\begin{array}{l}\text { Sunnyside(b) } \\
\text { Yakima(b) }\end{array}$ & $\begin{array}{l}14 \\
15\end{array}$ & $\begin{array}{l}0 \\
0\end{array}$ & $\begin{array}{l}\text { Ambient Dose, } \mathrm{DOH}^{(c)} \\
\text { Ambient Dose, } \mathrm{DOH}(c)\end{array}$ & \\
\hline
\end{tabular}

(a) Refer to Figure 7.1. Thermoluminescent Dosimeter (TLD) Locations on the Hanford site.

(b) Located at air sampling station.

(c) Mashington State Department of Heal th TLDs also at these locations.

(d) Refer to Figure 7.2, Thermoluminescent Dosimeter (TLD) Locations for Perimeter and Community sites. (e) Communi ty-Operated Énvi ronnental Surveillance Station. 


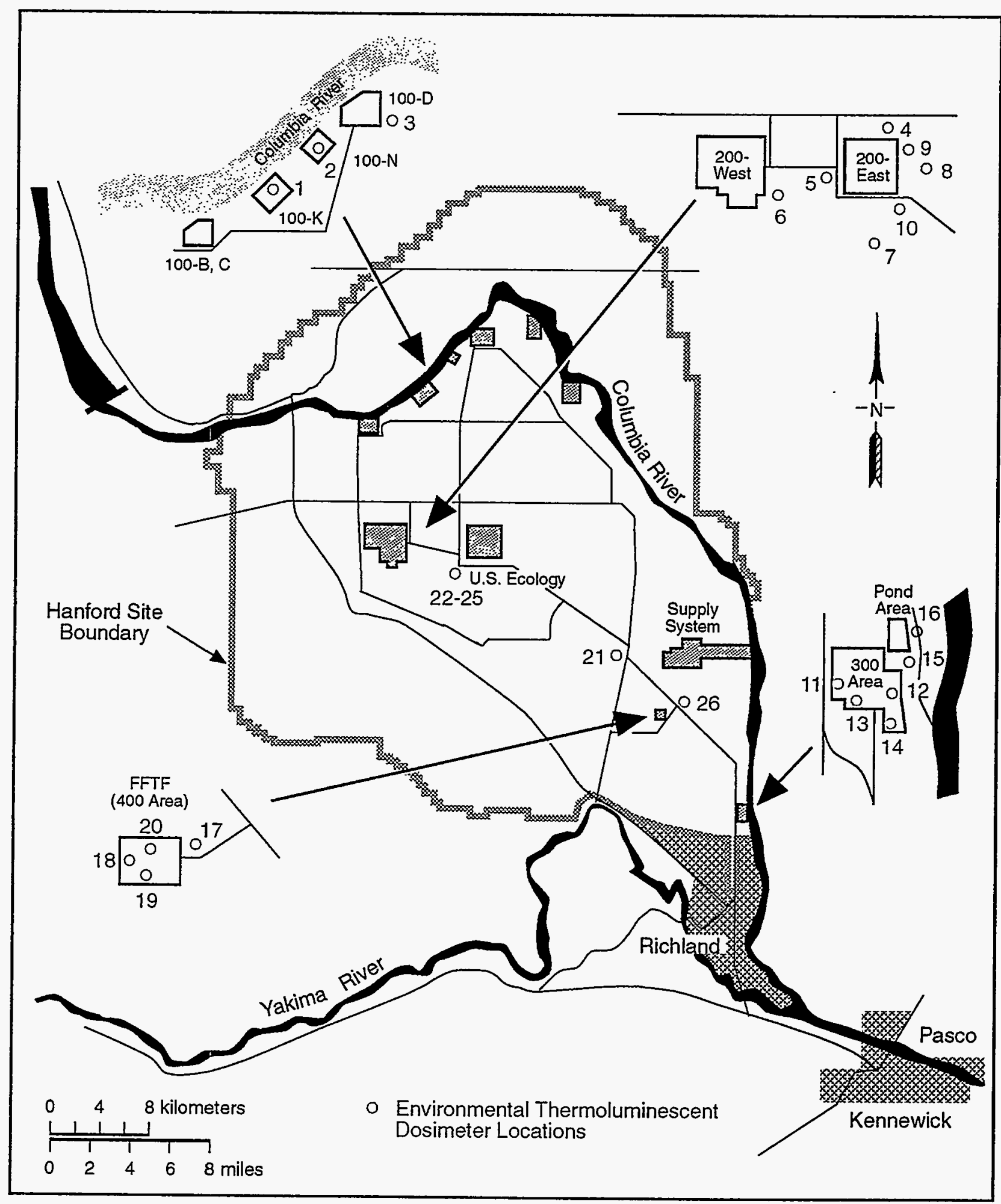

S9412049.6

Figure 7.1. Thermoluminescent Dosimeter (TLD) Locations on the Hanford Site 


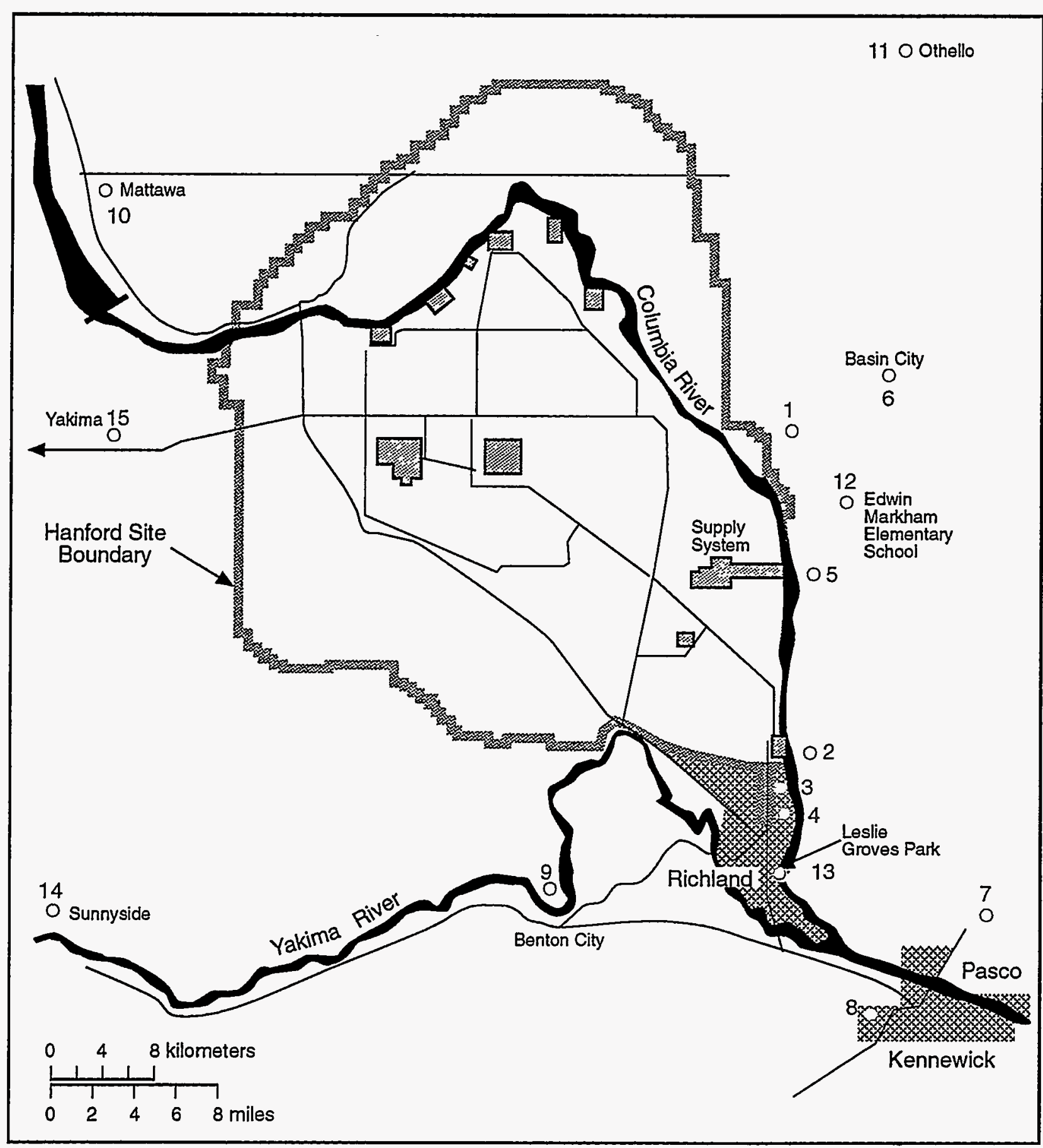

S9412049.3

Figure 7.2. Thermoluminescent Dosimeter (TLD) Locations for Perimeter and Community Sites 


\subsubsection{Columbia River Shoreline Locations}

\begin{tabular}{|c|c|c|c|}
\hline Location (a) & $\begin{array}{l}\text { Location } \\
\text { Number } \\
\end{array}$ & Frequency & Measurement \\
\hline Above 100 B Area & 1 & $Q$ & Ambient Dose \\
\hline Below 100 B Ret Basin & 2 & $\mathbf{Q}$ & Ambient Dose \\
\hline Above 1K Boat Ramp & 3 & $\mathbf{Q}$ & Ambient Dose \\
\hline Below 100N Outfall & 4 & M & Ambient Dose \\
\hline Below 100N stack & 5 & M & Ambient Dose \\
\hline Above Tip 100N Berm & 6 & $\boldsymbol{H}$ & Ambient Dose \\
\hline $100 \mathrm{~K}$ Trench Spring & 7 & $\mathbf{M}$ & Ambient Dose \\
\hline Below 100 D Area & 8 & $Q$ & Ambient Dose \\
\hline Belou Opp 100 D & 9 & $Q$ & Ambient Dose \\
\hline Lo End Locke Isl & 10 & Q & Ambient Dose \\
\hline White Bluffs Fy Lnd. & 11 & $Q$ & Ambient Dose \\
\hline White Bluffs slough & 12 & $\mathbf{Q}$ & Ambient Dose \\
\hline 100 F Floodplain & 13 & $Q$ & Ambient Dose \\
\hline Below $100 \mathrm{~F}$ & 14 & $\mathbf{Q}$ & Ambient Dose \\
\hline Hanford slough & 15 & $Q$ & Anbient Dose \\
\hline Hanf Powerl ine Xing & 16 & $Q$ & Ambient Dose \\
\hline Hanford Ferry Lnd & 17 & $\mathbf{Q}$ & Ambient Dose \\
\hline Hanford RR Track & 18 & $Q$ & Ambient Dose \\
\hline Savage Isl Slough & 19 & $Q$ & Ambient Dose \\
\hline Ringold Island & 20 & Q & Ambient Dose \\
\hline Powerline Crossing & 21 & $Q$ & Ambient Dose \\
\hline N End Hooded Island & 22 & $Q$ & Ambient Dose \\
\hline$S$ End Hooded Island & 23 & Q & Ambient Dose \\
\hline Island Hear 300 Area & 24 & $Q$ & Ambient Dose \\
\hline Is I DS Bateman Isl & 25 & $Q$ & Ambient Dose \\
\hline Port of Benton-River & 26 & $Q$ & Ambient Dose \\
\hline $100-0$ Is land & 27 & $\mathbf{Q}$ & Ambient Dose \\
\hline Is Ind Above 300 Area & 28 & $\mathbf{Q}$ & Ambient Dose \\
\hline
\end{tabular}

(a) Refer to Figure 7.3, Thermoluminescent Dosimeter (TLD) Locations on the Hanford Reach of the Columbia River. 


\subsection{COLUMBIA RIVER SHORELINE RADIATION SURVEYS}

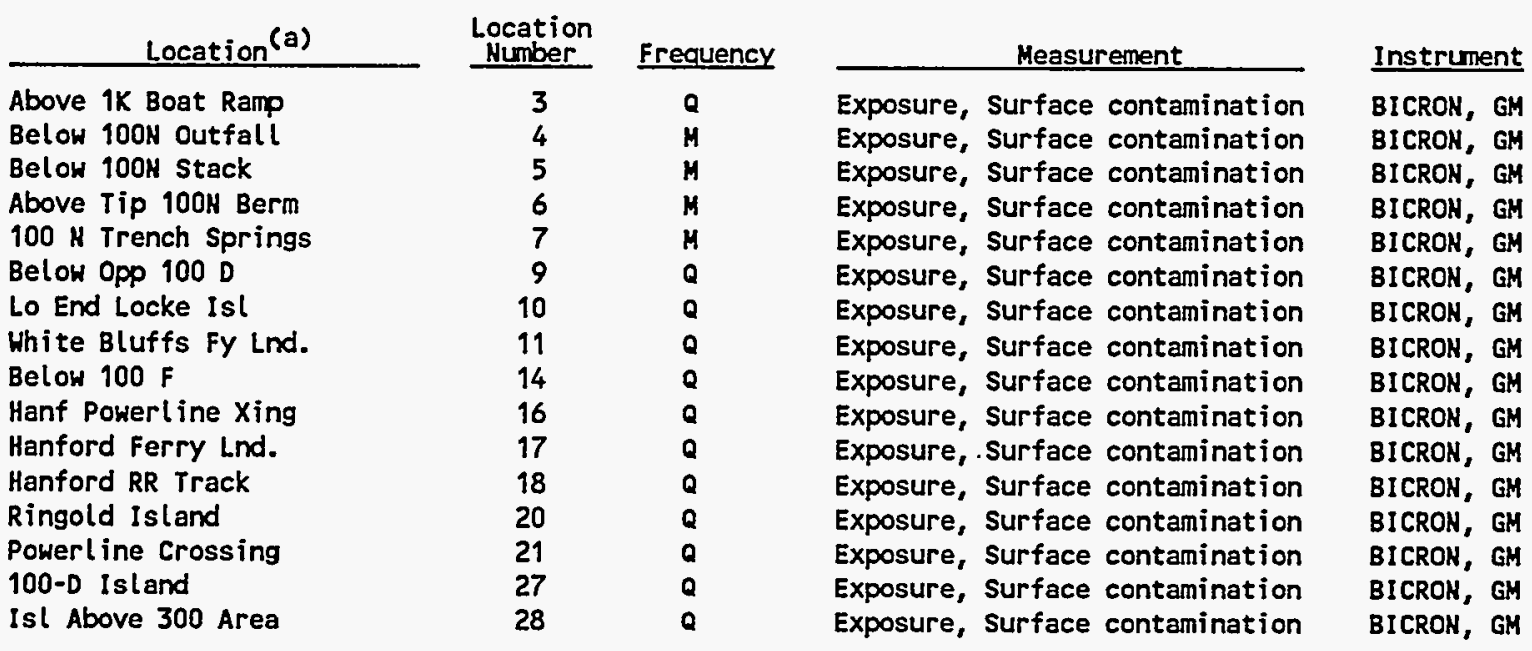

(a) Refer to Figure 7.3, Thermoluminescent Dosimeter (TLD) Locations on the Hanford Reach of the Columbia River. 


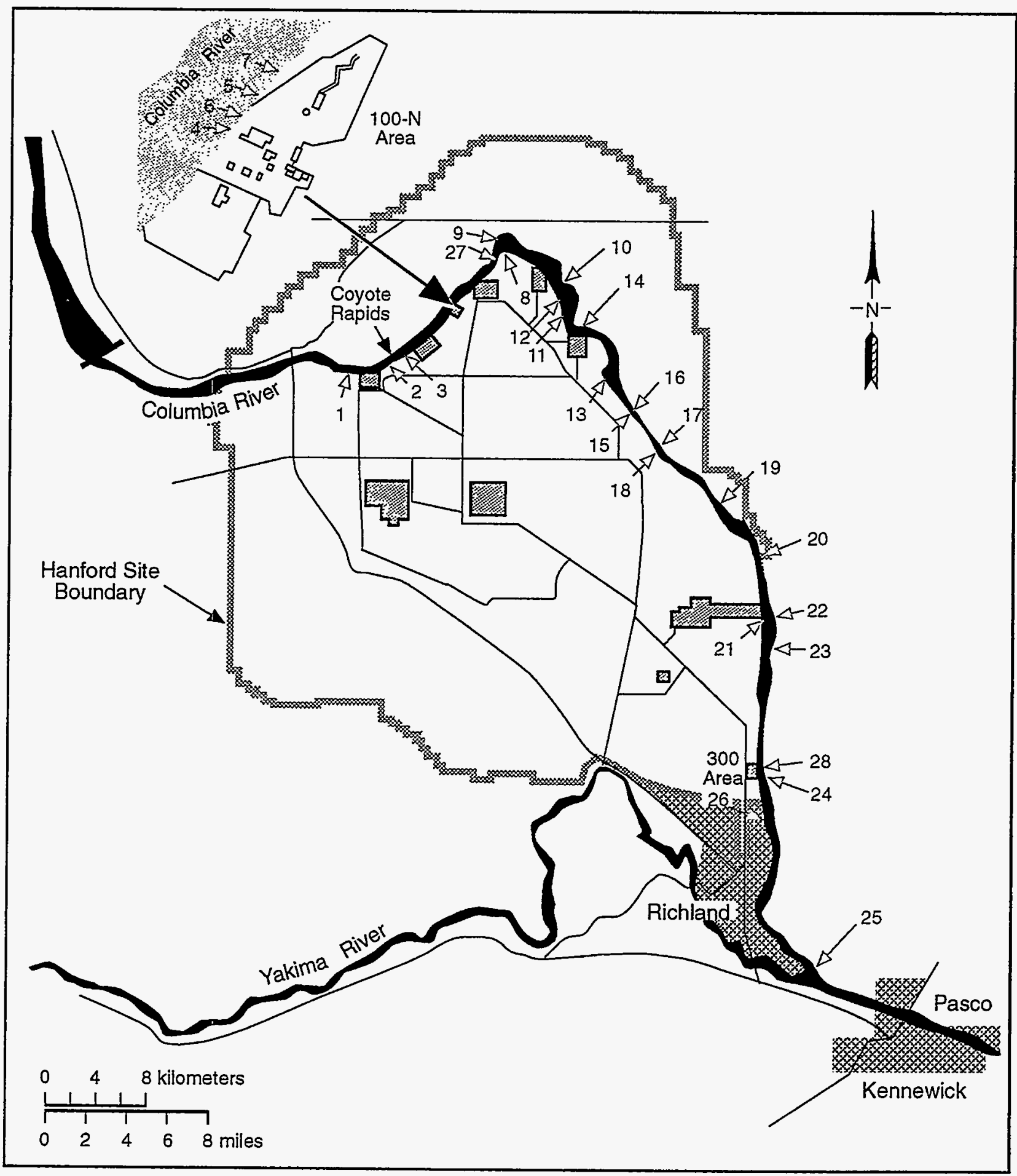

Figure 7.3. Thermoluminescent Dosimeter (TLD) Locations on the Hanford Reach of the Columbia River 
PART II. HANFORD GROUND-HATER SURVEILLANCE 



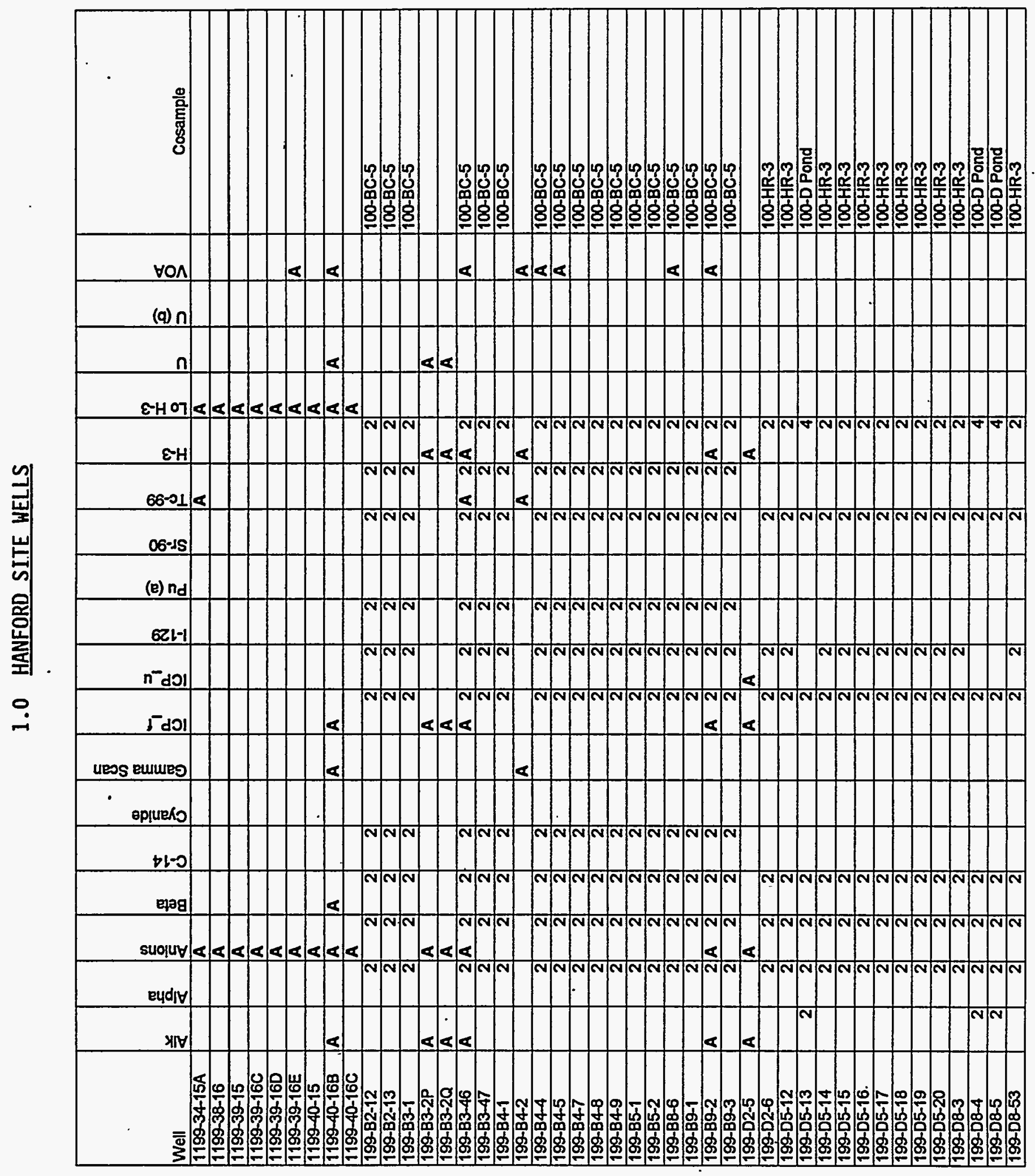




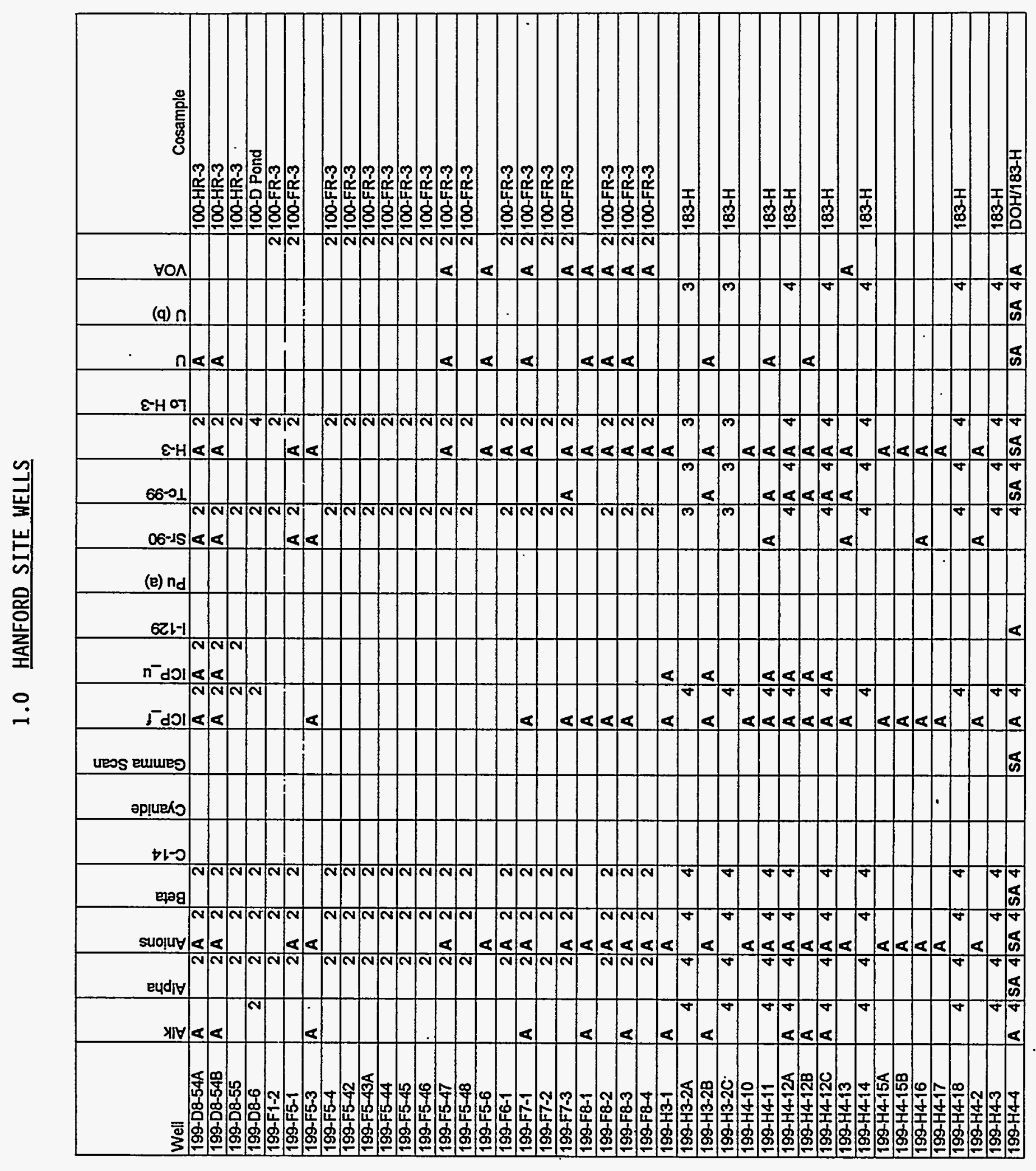




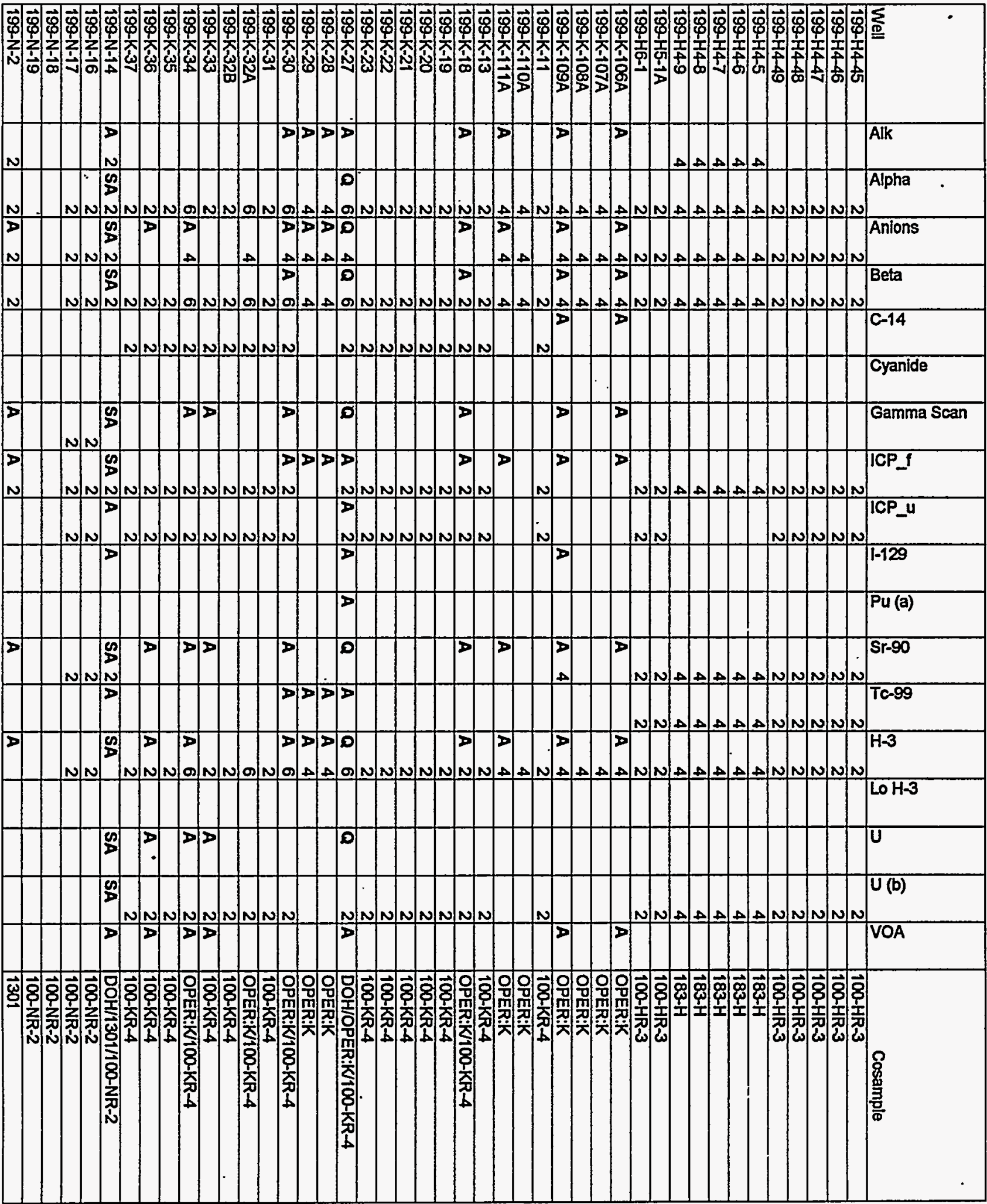




\subsection{HANFORD SITE WELLS}

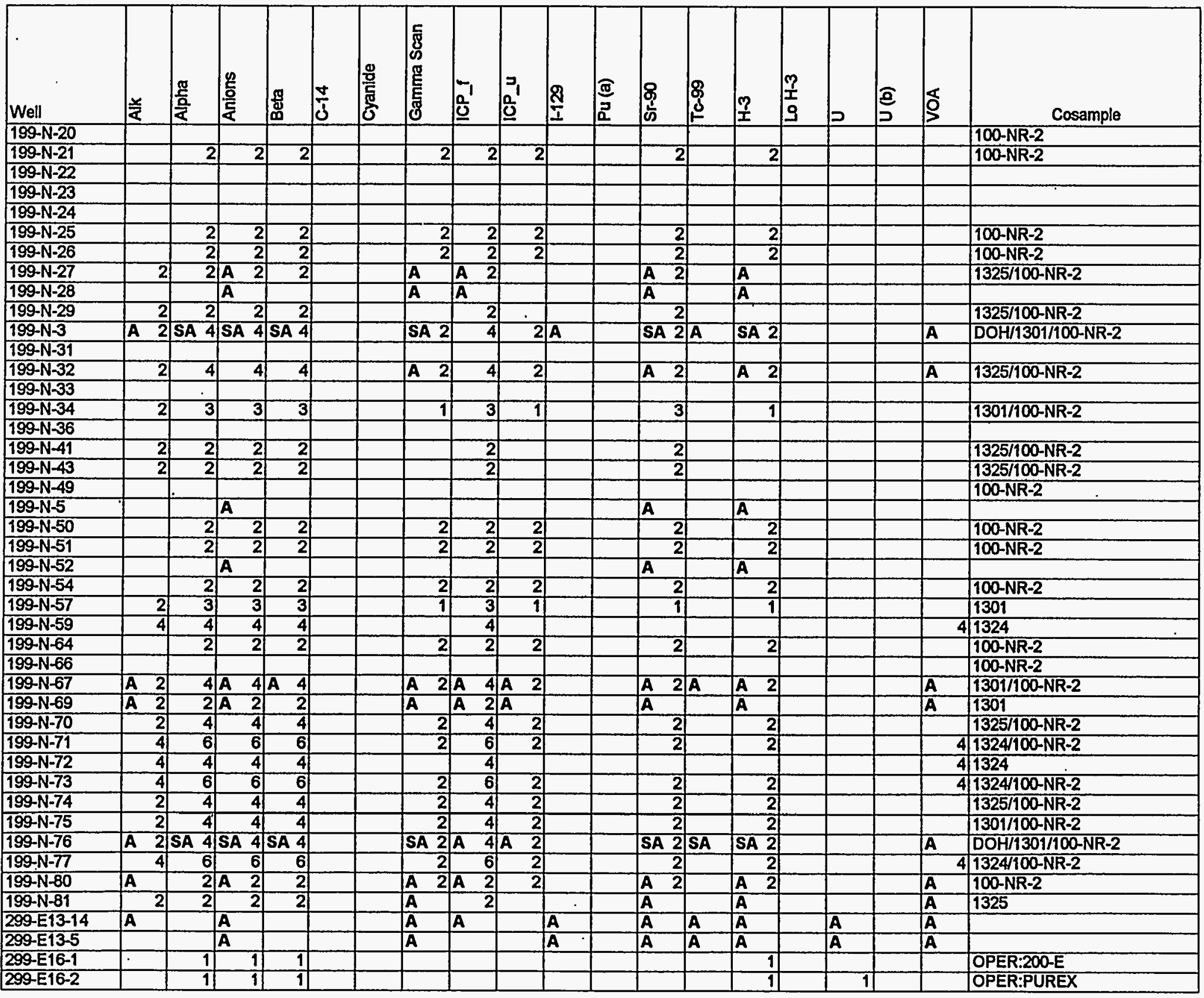




\begin{tabular}{|c|c|c|c|c|c|c|c|c|c|c|c|c|c|c|c|c|c|c|c|}
\hline xaynd:yבdO & & & 1 & & $b$ & & & & & & & & & & 1 & $b$ & $l$ & & $62-923-662$ \\
\hline & & & & & $\forall$ & & & & $\forall$ & & & & & & & $\boldsymbol{\nabla}$ & & : & $82-923-662$ \\
\hline xaynd:yבzdo & & & $l$ & & $b$ & & & & $\mathbf{v}$ & & & & & & $b$ & $b$ & $l$ & & $2 z-923-662$ \\
\hline $6 z-\forall-912$ & & & & & VI & & & & $\forall$ & $b$ & $t$ & & & & & 6 & & $b$ & $12-923-662$ \\
\hline $6 z-6-9 \mid z$ & & & & & & & & & & 6 & $b$ & & & & & 6 & & $b$ & $02-923-662$ \\
\hline $6 z-\forall-912$ & & & & & & & & & & $b$ & $t$ & & & & & $t$ & & $b$ & $61-923-662$ \\
\hline $6 Z-\forall-9 \mid Z$ & & & & & ज & & & & $\forall$ & $b$ & $\bar{b}$ & & & & & t & & t & $81-G Z \exists-66 Z$ \\
\hline 3-00z:y $3 \mathrm{dO}$ & & & & & $\begin{array}{ll}1 & \forall \\
\end{array}$ & & & & $\forall$ & & & & & & I & 1 & $b$ & & $\angle 1-923-662$ \\
\hline$x \exists y \cap d: y \exists d O / 6 z-\forall-91 z$ & & & 1 & & $\begin{array}{ll}1 \quad \forall \\
\end{array}$ & & & & $\forall$ & 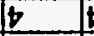 & t & & & & 1 & 9 & 1 & $t$ & $11-923-662$ \\
\hline & & & & & & & & & & & & & & & & & $L$ & - & $0001-923-662$ \\
\hline & & & & & (V) & & & & ( & & & ( & & & ( & $\mathbf{v}$ & & & $8+27-662$ \\
\hline$\exists-00 z: y \exists d O$ & & & & & $l$ & $\forall$ & & & $\mathbf{v}$ & & & & & & 1 & $\ell$ & $l$ & & $\angle+2 \exists-662$ \\
\hline$\exists-002: y \exists d 0$ & & & & & 1 & & & & $\mathbf{V}$ & & & & & & 1 & 1 & 1 & & $9+27-662$ \\
\hline $3-00 z: 4 \exists d 0$ & & & & & 1 & & & & ( & & & & & & 1 & 1 & 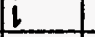 & & $\forall+23-662$ \\
\hline$x \forall-\forall-\perp S S$ & & & 1 & & 2 & $z$ & & & $z$ & & 1 & & & & $\bar{z}$ & I & $\bar{z}$ & 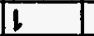 & $0 z+27-662$ \\
\hline $0 t-\forall \div 9 \mid Z$ & & & & & $z$ & & & & & & I & & & & 2 & 6 & $z$ & $b$ & $z-273-66 z$ \\
\hline$\exists-00 z: y \exists d 0 / x \forall-\forall-1 S S$ & & & 1 & & $\varepsilon$ & $z$ & & & 2 & & 1 & & & & $\varepsilon$ & $Z$ & $\varepsilon$ & 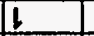 & $61+23-662$ \\
\hline $89 \varepsilon-\forall-9 l Z / O L-\forall-9 \mid Z$ & & & & & 9 & $\mathbf{v}$ & v & & $\mathbf{\forall}$ & & $E$ & & & & 9 & $\varepsilon$ & 9 & $\varepsilon$ & $81+2 \exists-66 z$ \\
\hline $01-\forall-91 Z$ & & & & & 2 & & $\boldsymbol{\theta}$ & & I. & & I & & & & $\bar{z}$ & I & $\bar{z}$ & $\bar{l}$ & $\angle 1+22 \exists-662$ \\
\hline $01-\forall-912$ & & & & & $z$ & & $\forall$ & & $\forall$ & & I & & & & $z$ & I & $z$ & $l$ & $91-+23-662$ \\
\hline & & & & & $\forall$ & $\bar{v}$ & $\forall$ & & 甘 & & 1 & घ) & & & $\mathbf{v}$ & $\mathbf{v}$ & $\mathbf{U}$ & & $\varepsilon 1+23-662$ \\
\hline & & & & & $\forall$ & & $\forall$ & & $\forall$ & & 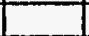 & & & & $\forall$ & 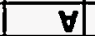 & & & $21+23-662$ \\
\hline & & & & & $\forall$ & & & & $\forall$ & & & & & & & $\boldsymbol{\nabla}$ & & 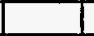 & $11+2 \exists-66 z$ \\
\hline & & & & & $\forall$ & & $\boldsymbol{\theta}$ & & $\forall$ & & & & & & ( & $\mathbf{\forall}$ & & & $1+27-662$ \\
\hline$\exists-00 z: y \exists d 0$ & & & & & $b$ & & & & () & & & & & & 1 & I & $t$ & & $1-\varepsilon 2 \exists-66 z$ \\
\hline puod W-1012 & & & & . & & & & & & & 2 & & & & $z$ & 2 & $\bar{z}$ & $\bar{z}$ & $-813-662$ \\
\hline puod W-lOLZ & & & & & & & & & & & Z & & & & 2 & 2 & $z$ & $z$ & $\varepsilon-81=66 z$ \\
\hline puod w-lol2 & & & & & & & & & & & $z$ & & & & $z$ & 2 & $z$ & $z$ & $z-81 \exists-66 z$ \\
\hline puod $w$-lolz/puod-g:yjdO & & & & & $1 \quad \forall$ & & & & $\forall$ & & 6 & & & & 9 & 9 & 9 & 9 & $1-81 \Xi-662$ \\
\hline $89 \varepsilon-\forall-91 Z$ & & & & & $2 \quad \forall$ & & $\mathbf{v}$ & & ( & & II & & & $\forall$ & 2 & 1 & $z$ & 1 & $6-\angle 1 \exists-662$ \\
\hline & & & & & $\forall$ & & $\forall$ & & $\forall$ & & & & & & $\forall$ & 8 & & & $8-\angle 1 \exists-662$ \\
\hline & & & $\mathbf{y}$ & & $\forall$ & & ( & & $\forall$ & & & $\forall$ & & & 甘 & 甘] & & & $9-213-662$ \\
\hline $89 \varepsilon-\forall-912$ & & & & & $\bar{z}$ & $\mathbf{\theta}$ & $\mathbf{\theta}$ & & $\forall$ & & I & & & & $z$ & $t$ & $\bar{z}$ & I & 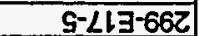 \\
\hline $0 l-\forall-9 l Z / 6 Z-\forall-9 l Z$ & & & & & $\begin{array}{ll}2 & \forall\end{array}$ & & 8 & & $\begin{array}{ll}\mathbf{l} & \mathbf{U}\end{array}$ & $b$ & $s$ & & & & $2 \quad$ & $9 \quad \forall$ & $\bar{z}$ & $\mathrm{~s}$ & $02-\angle 13-662$ \\
\hline & & & & & $\mathbf{v}$ & & $\forall$ & & $\forall$ & & & & & & $\forall$ & v & & & $z-\angle 13-662$ \\
\hline $0 l-\forall-9 l z$ & & & & & 2 & & 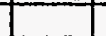 & & & & I & & & & $\bar{z}$ & 1 & $\bar{z}$ & l & $61-\angle 1 \exists-662$ \\
\hline $89 \varepsilon-\forall-912$ & & & & & $\bar{z}$ & & & & $\nabla$ & & I & & & & $2 \forall$ & $1 \quad \forall$ & $\bar{z}$ & L & $81-\angle 1 \exists-66 Z$ \\
\hline 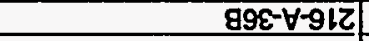 & & & & & $\begin{array}{ll}z & y\end{array}$ & & $\forall$ & & $\forall$ & & 1 & & & & 20 & $1 \quad \forall$ & $z$ & 1 & $\angle 1-\angle 1 \exists-66 Z$ \\
\hline $89 \varepsilon-\forall \cdot 91 z$ & & & & & $\begin{array}{ll}Z & \forall\end{array}$ & & $\boldsymbol{\forall}$ & & $\forall$ & & 1 & & & & $\begin{array}{l}2 \quad \forall \\
0\end{array}$ & $\begin{array}{ll}1 & 0\end{array}$ & $z$ & $b$ & $9 l-\angle L \exists-66 Z$ \\
\hline 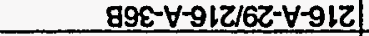 & & & & & $\begin{array}{ll}z & \forall\end{array}$ & $\forall$ & $\forall$ & & $\begin{array}{ll}1 & 0\end{array}$ & $z$ & $\varepsilon$ & & & & $\begin{array}{ll}z & \forall\end{array}$ & {$\left[\begin{array}{ll}\varepsilon & \boldsymbol{y}\end{array} \mid\right.$} & $z$ & $\varepsilon$ & SL- $\angle L \exists-662$ \\
\hline $89 \varepsilon-\forall-912$ & & & & & $z \quad \forall$ & & $\mathbf{v}$ & & $\nabla$ & & 1 & & & & $2 v$ & $1 \quad 8$ & $z$ & t. & $\nabla 1-\angle 1 \exists-662$ \\
\hline xaynd:yヨdo & & & & & 1 & & & & & & & & & & l & It & l & & $\varepsilon l-\angle L=-66 Z$ \\
\hline xaynd:yヨdo & & & & & 1 & & & & & & & & & & 1 & $t$ & 1 & & Zl-Ll $\exists-66 Z$ \\
\hline $01-\forall-9 l Z$ & & & & & $z$ & & $\forall$ & & छ & & 1 & & & & $z$ & 1 & $z$ & 1 & $1-\angle 13-662$ \\
\hline ө|duresoo & §) & 둥 & c & $\frac{5}{6}$ & $\overline{\mathbf{C}}$ & 8 & की & 롱 & $\overline{\overline{\mathrm{V}}}$ & $\begin{array}{l}\bar{\alpha} \\
\text { ' } \\
\end{array}$ & वิ & 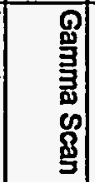 & $\begin{array}{l}\text { Q } \\
\text { : } \\
\text { 言 }\end{array}$ & $\frac{9}{A}$ & 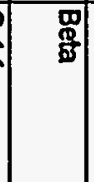 & 年 & 함 & D & $\| \theta M$ \\
\hline
\end{tabular}




\subsection{HANFORD SITE WELLS}

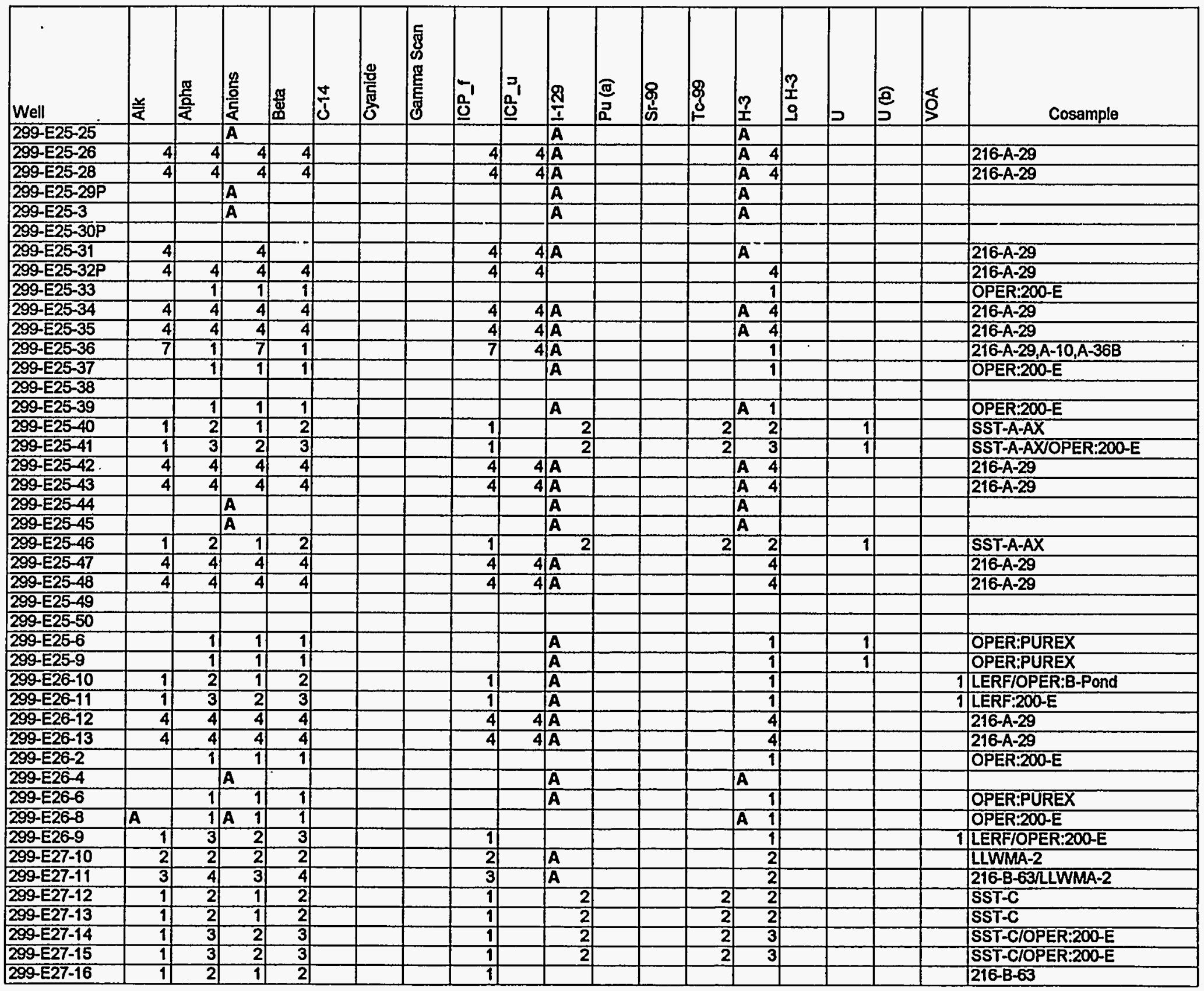




\subsection{HANFORD SITE HELLS}

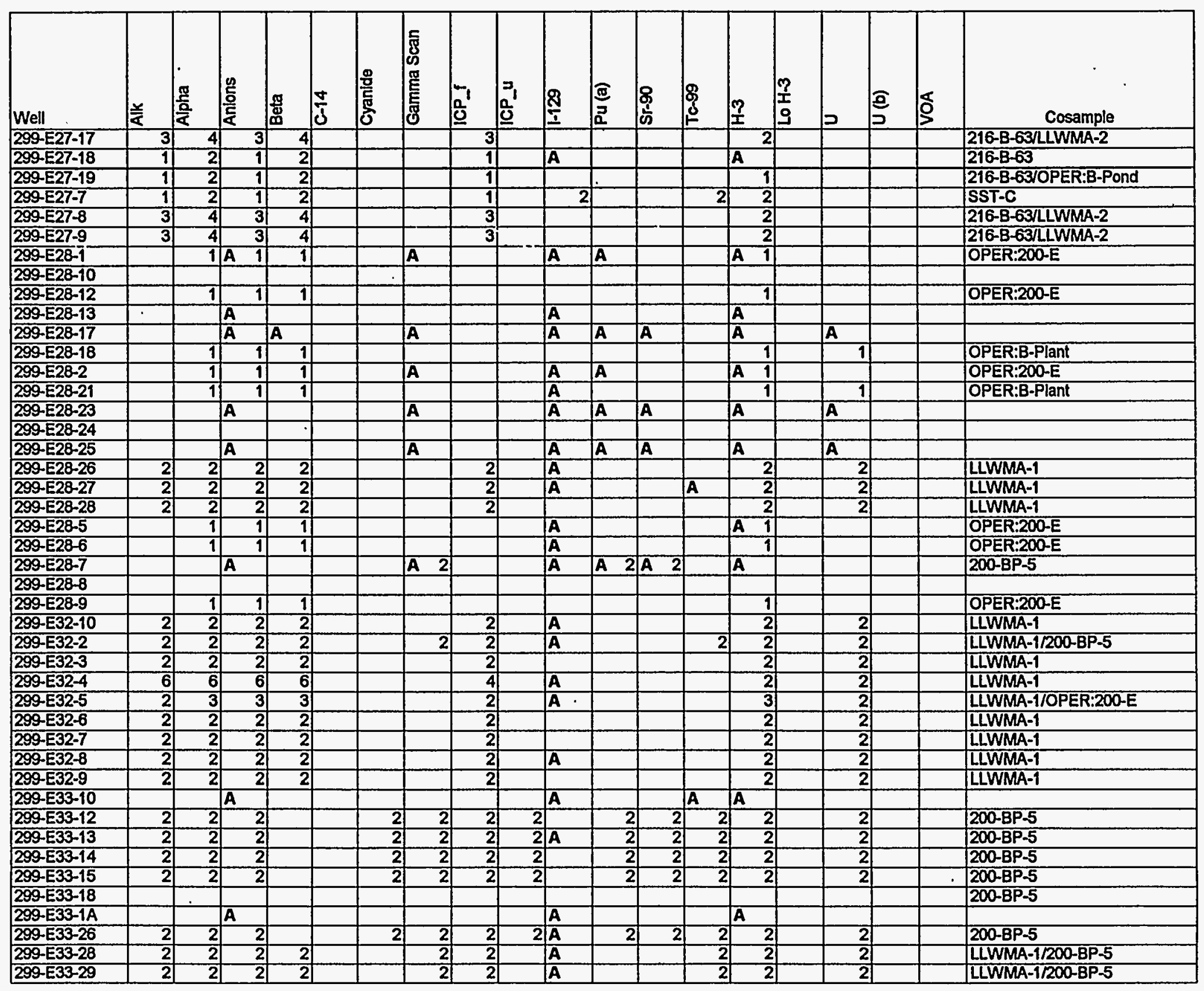




\subsection{HANFORD SITE WELLS}

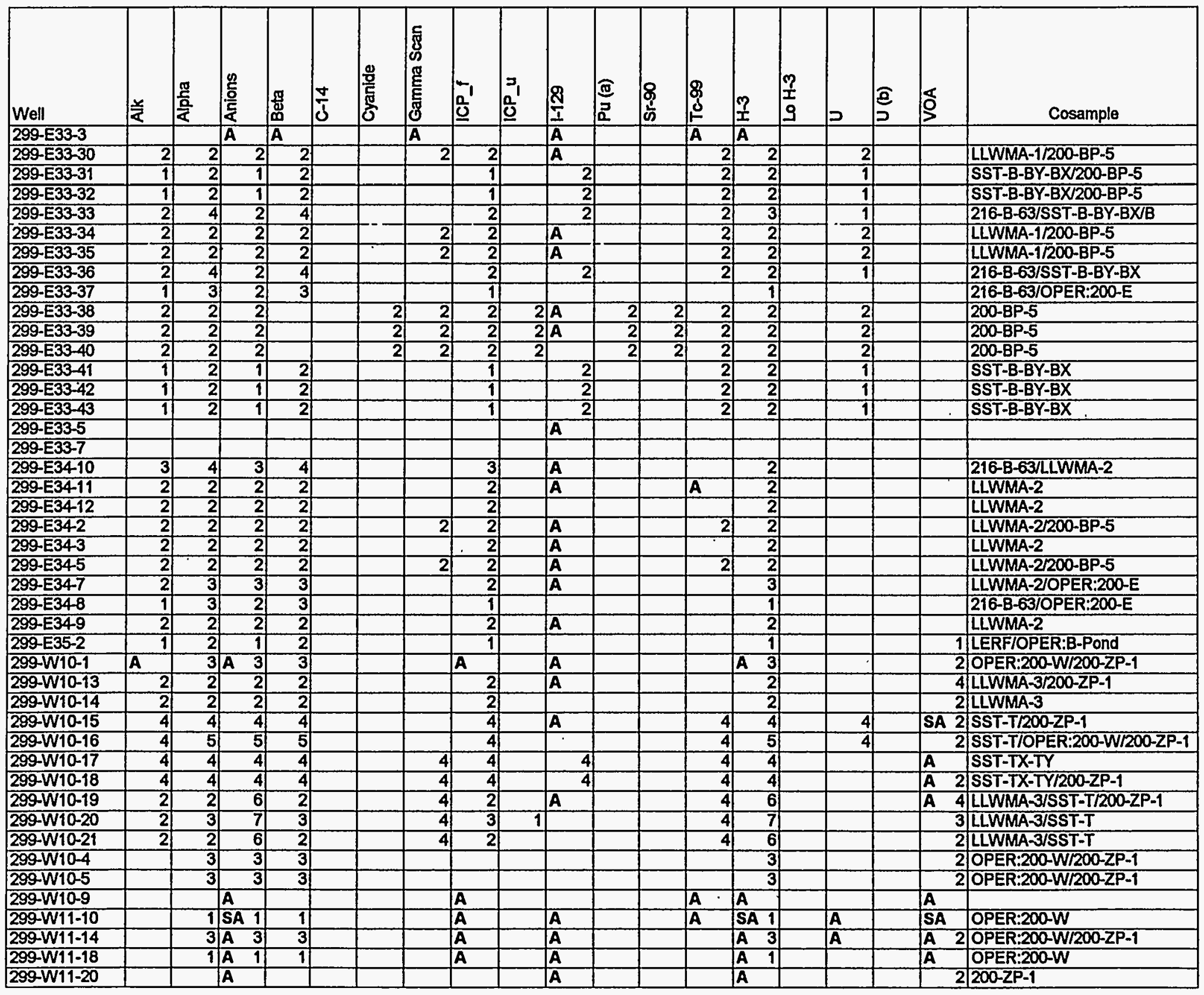




\subsection{HANFORD SITE HELLS}

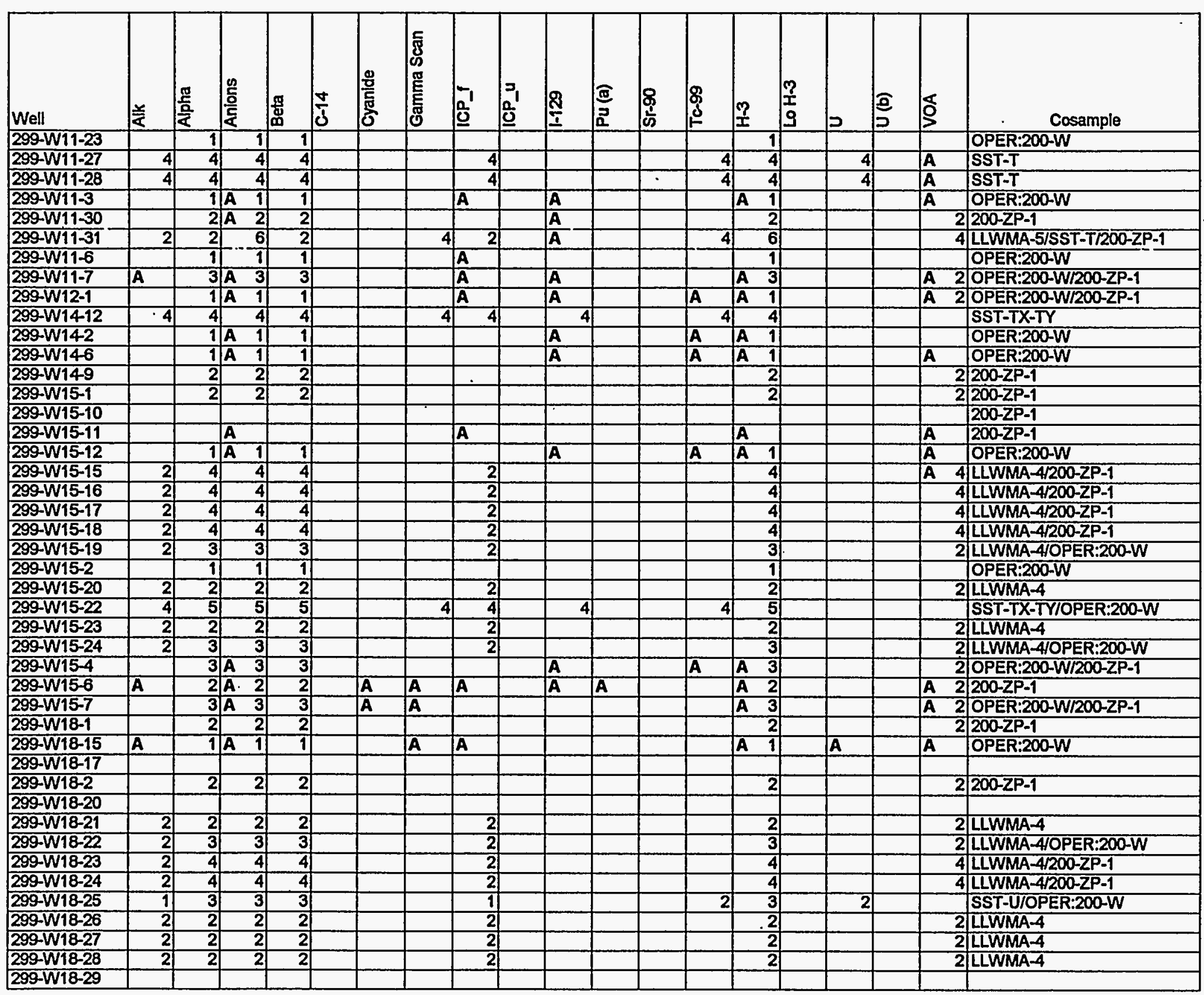




\subsection{HANFORD SITE WELLS}

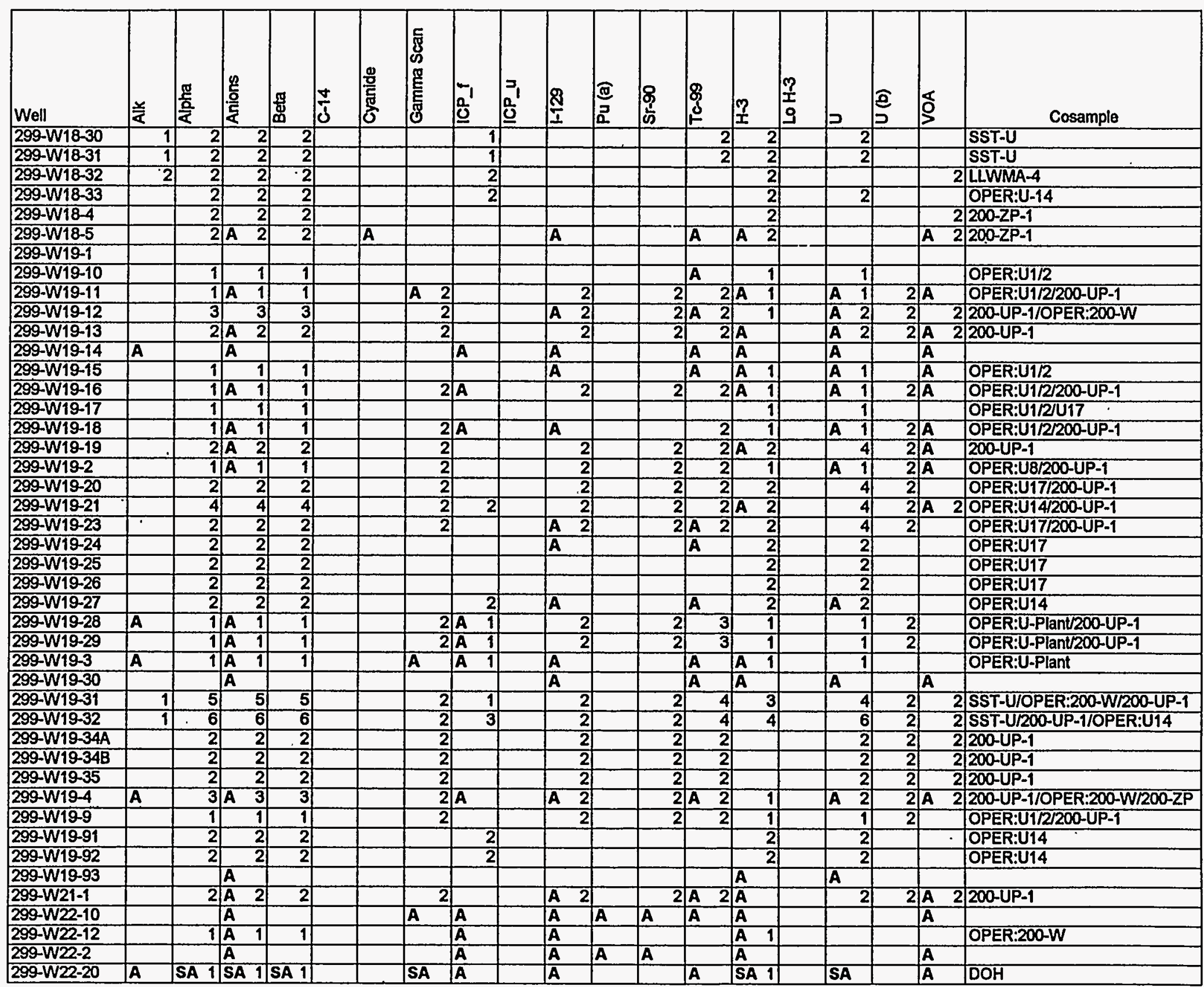




\subsection{HANFORD SITE HELLS}

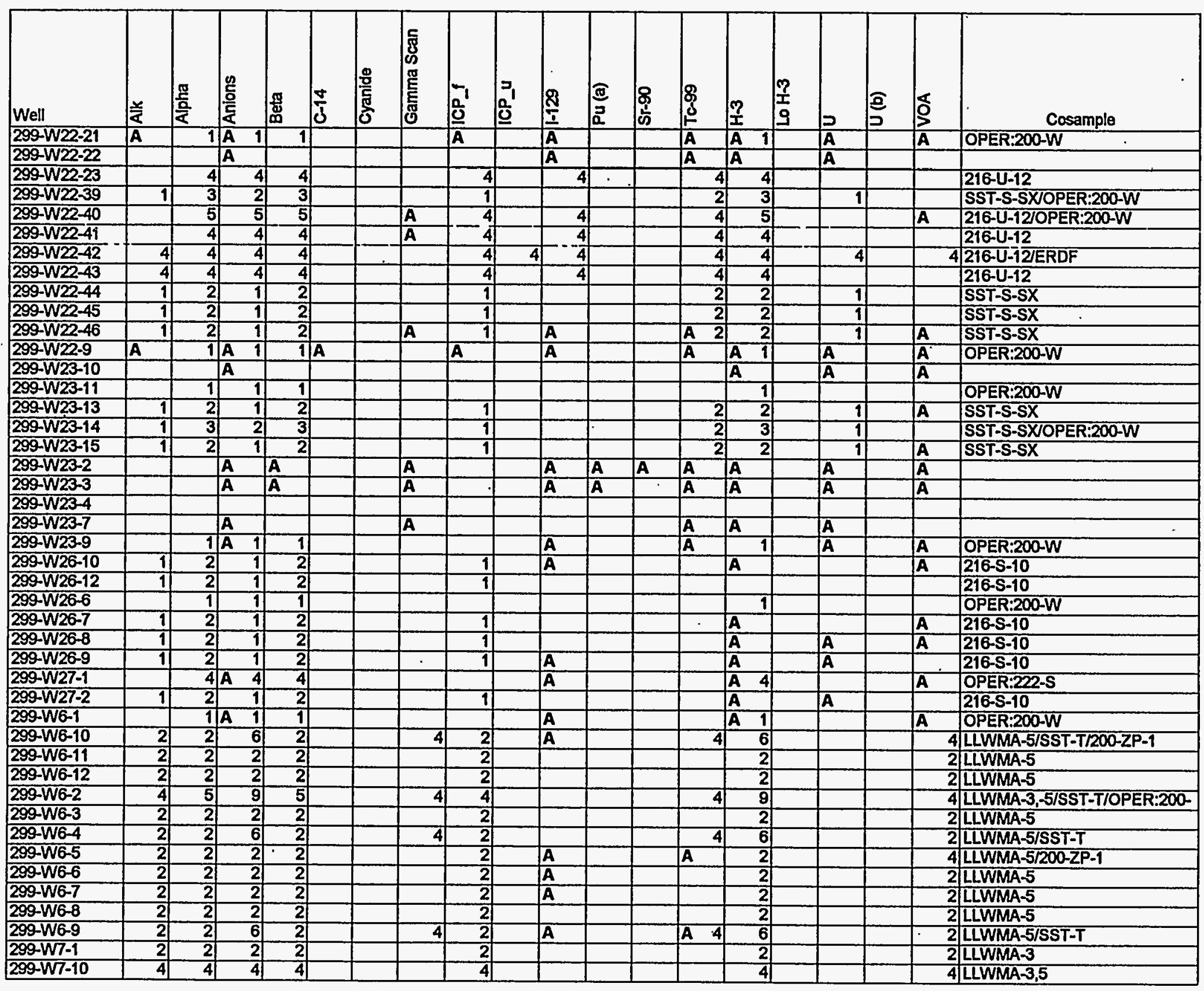




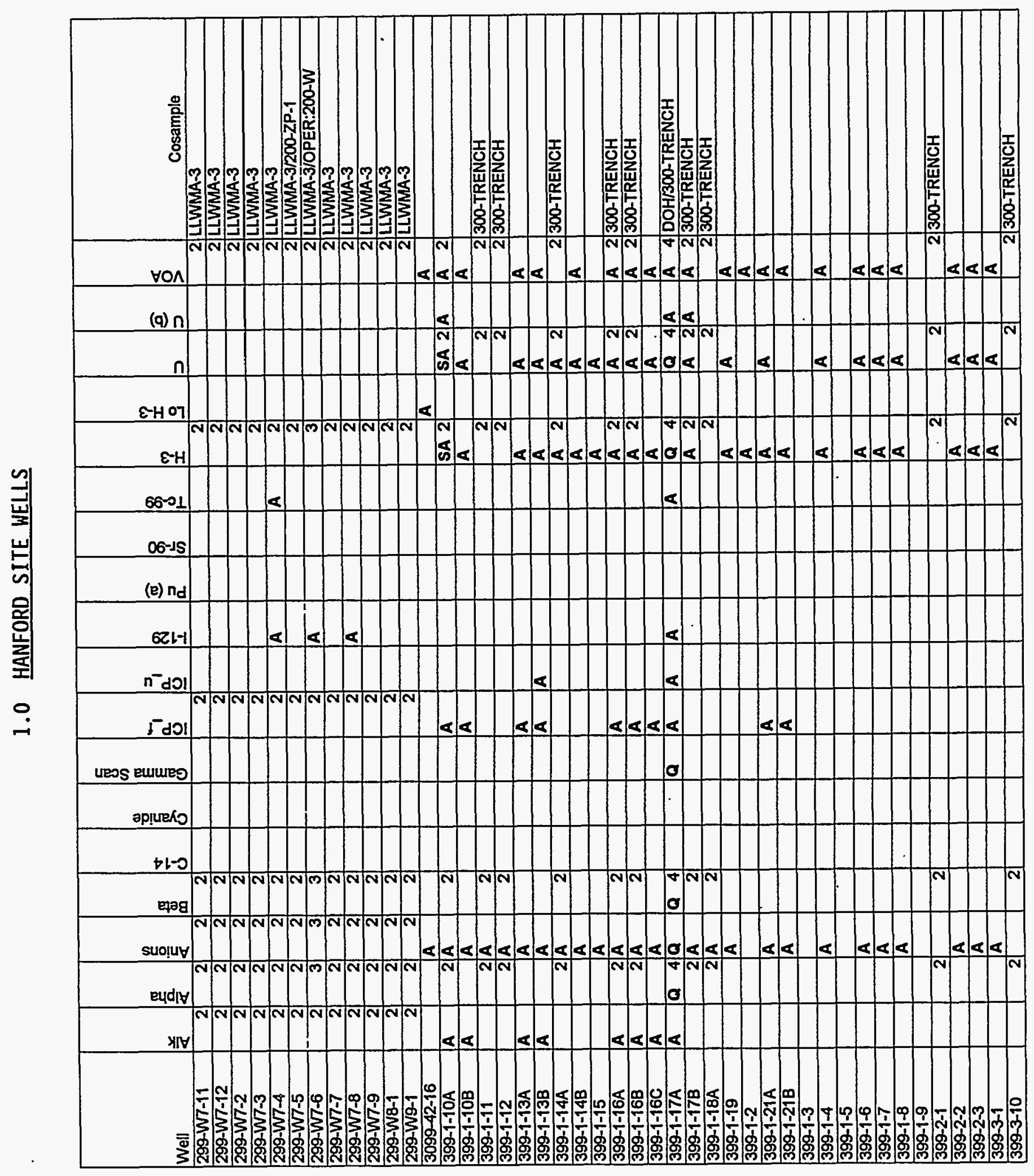




\subsection{HANFORD SITE WELLS}

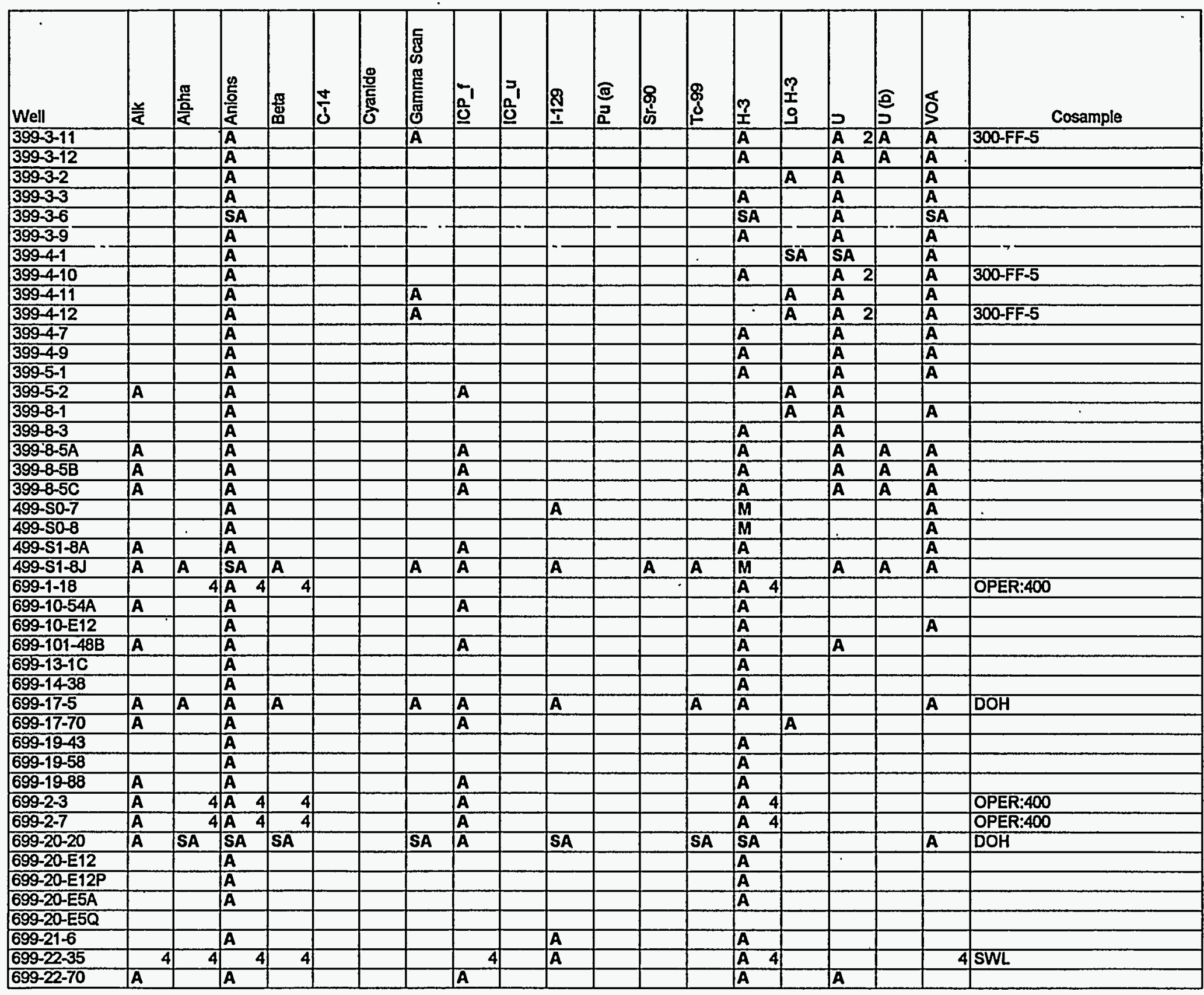




\subsection{HANFORD SITE WELLS}

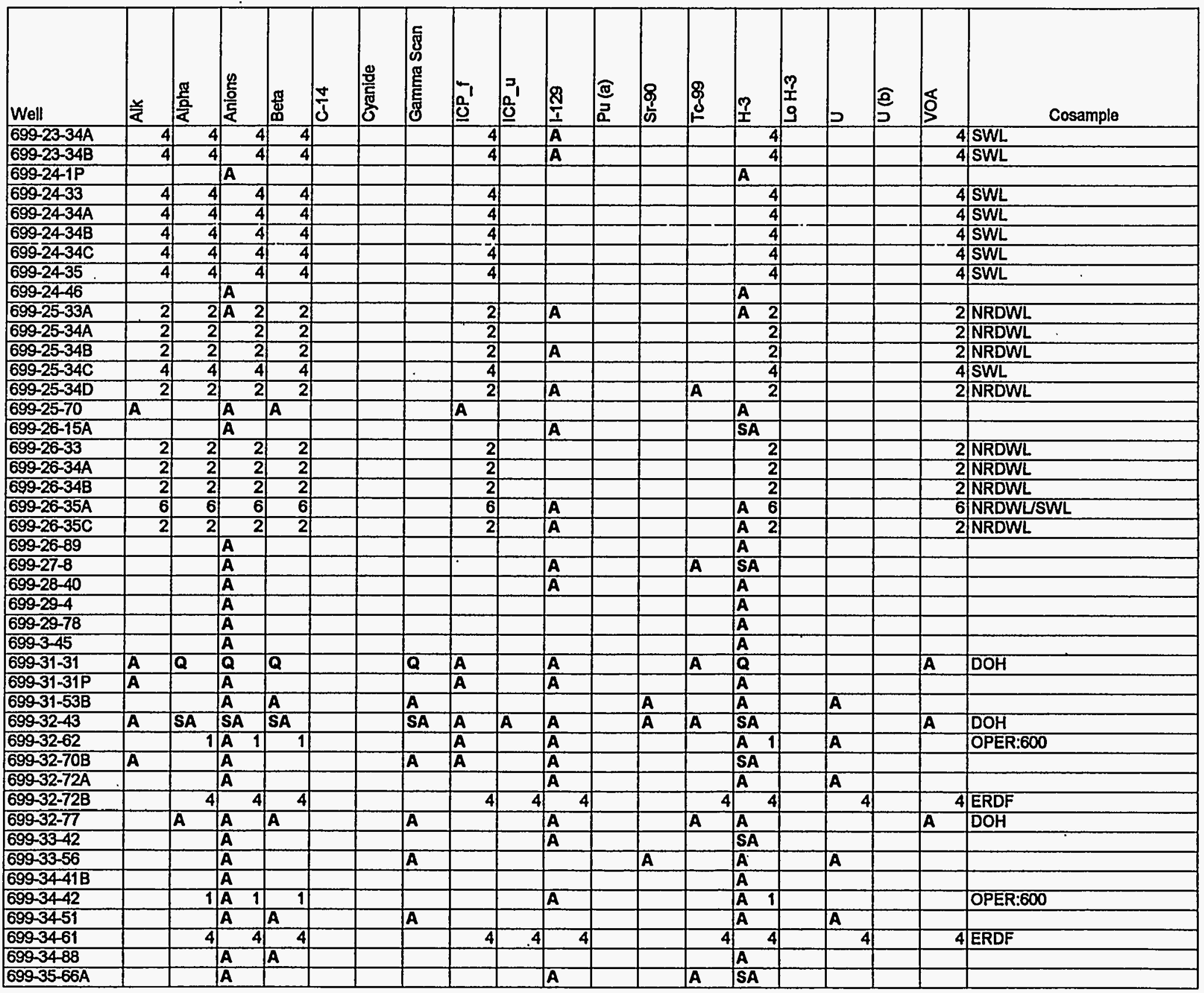




\subsection{HANFORD SITE WELLS}

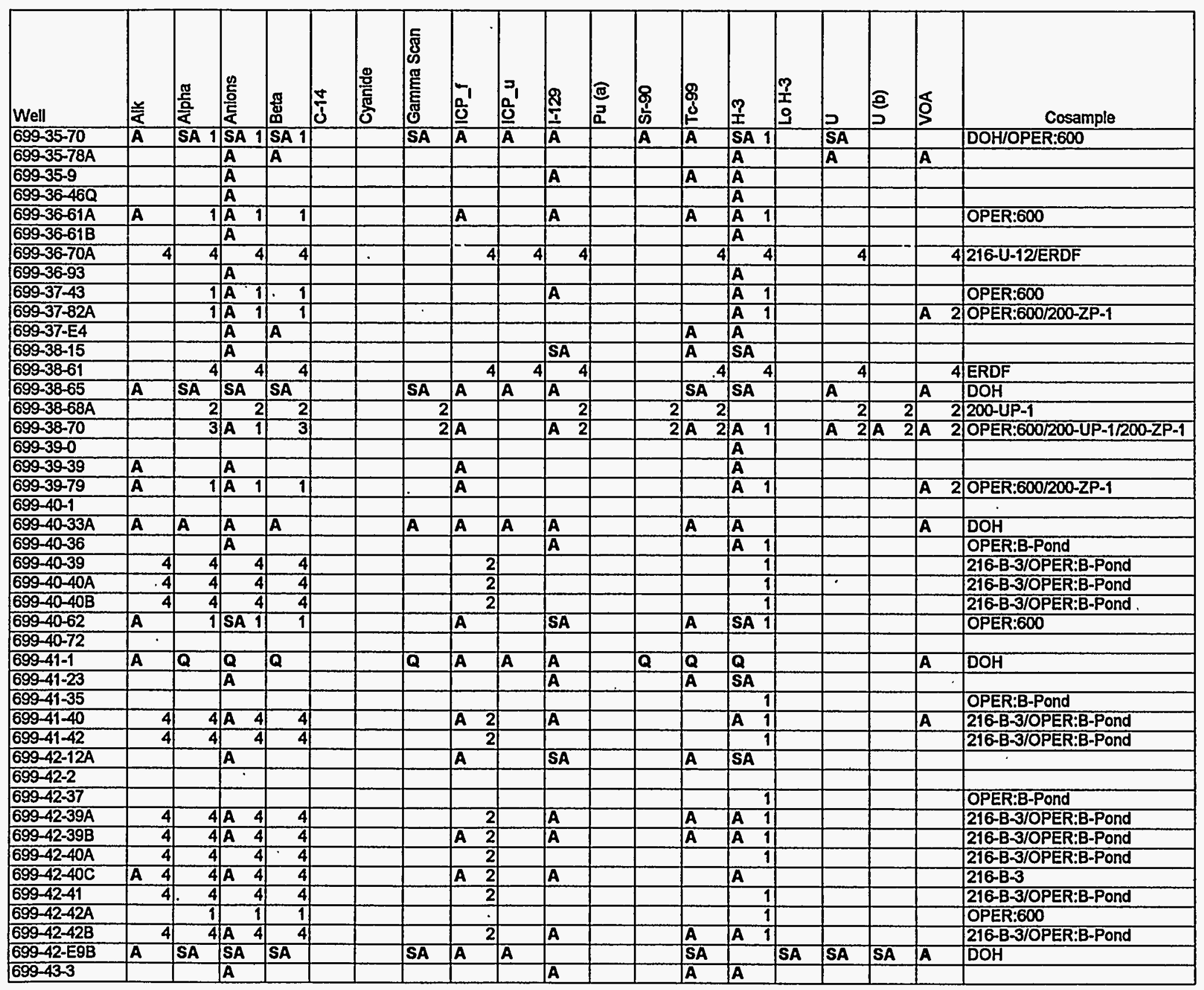




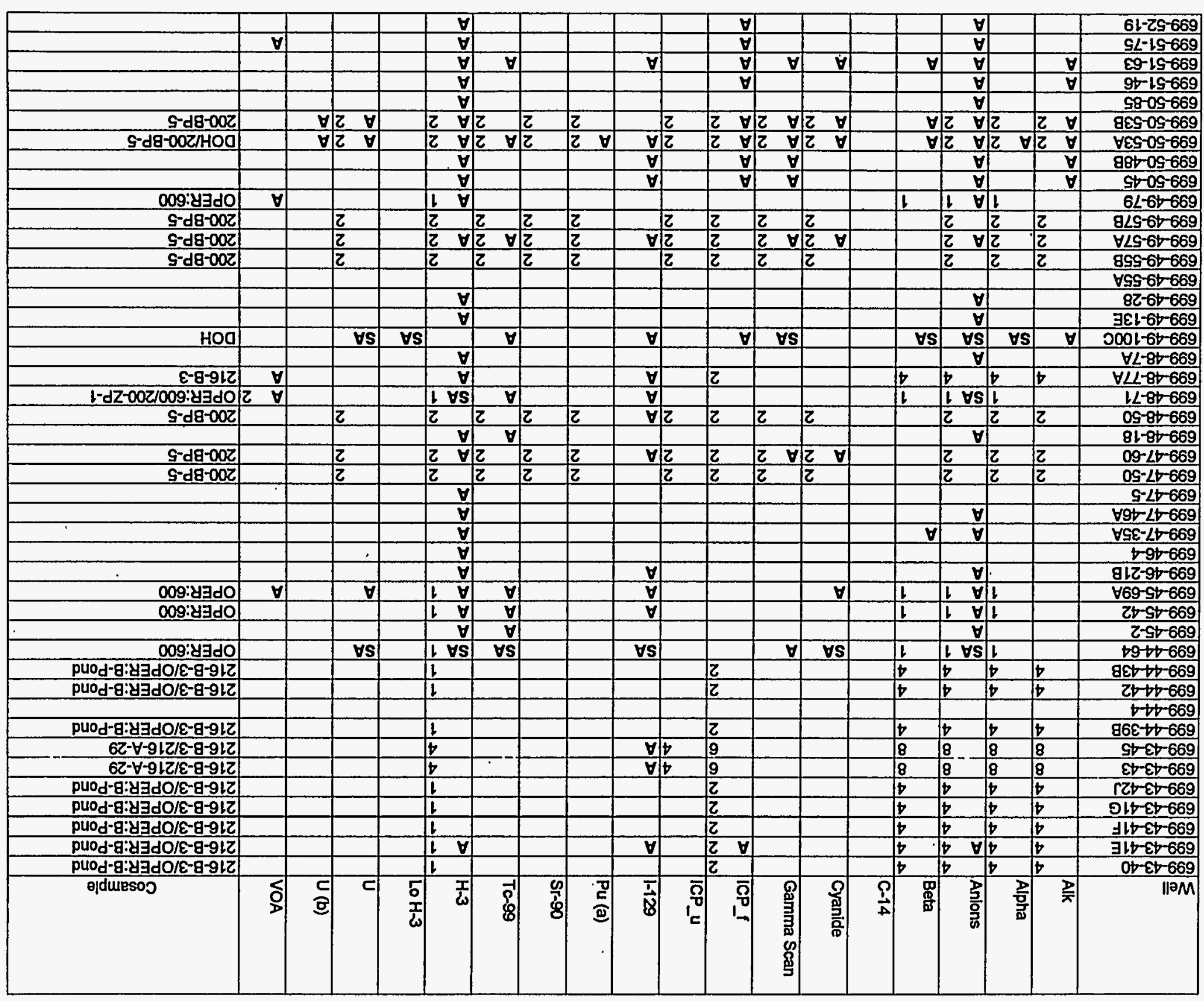




\subsection{HANFORD SITE WELLS}

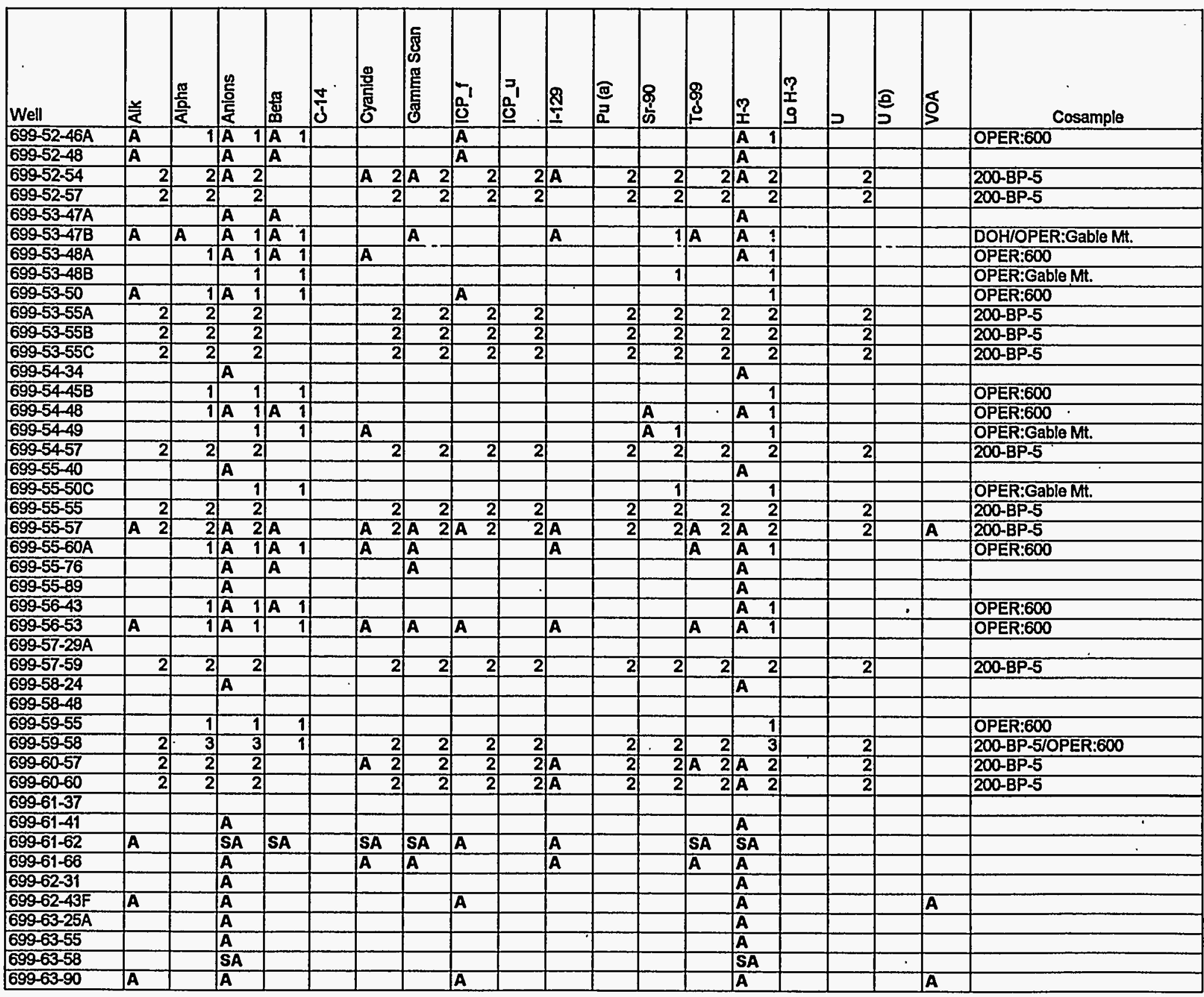




\subsection{HANFORD SITE WELLS}

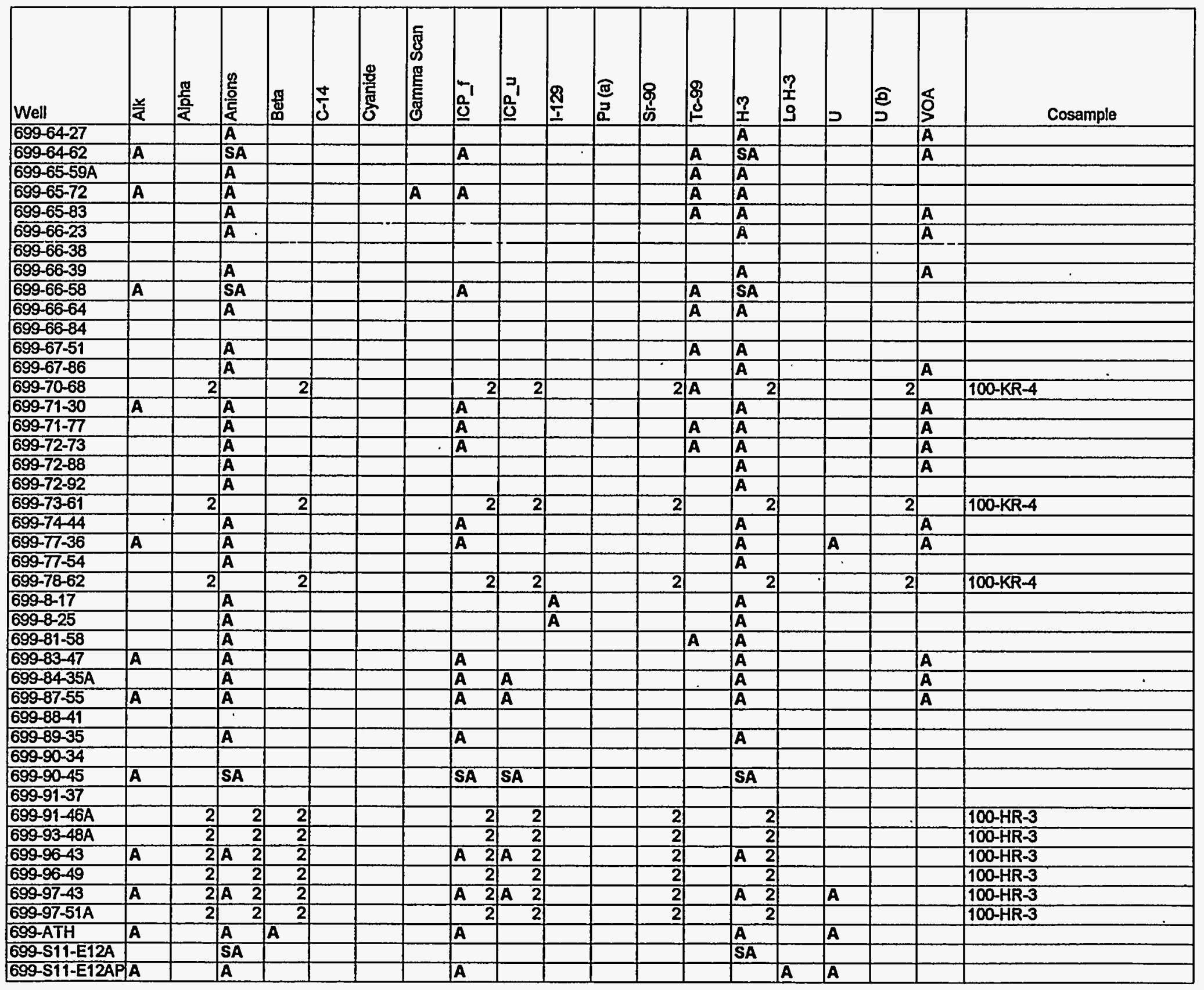




\subsection{HANFORD SITE WELLS}

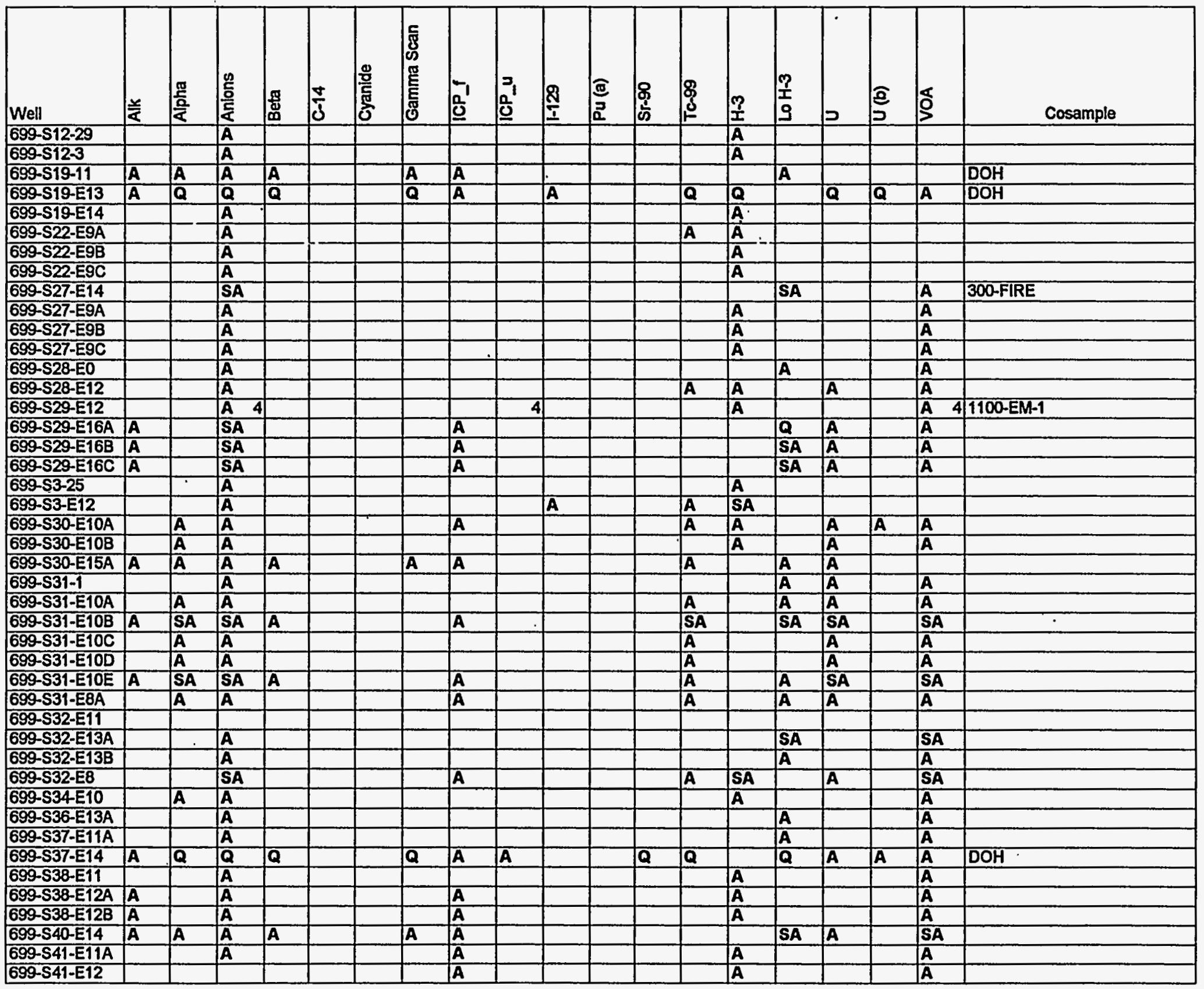




\subsection{HANFORD SITE WELLS}

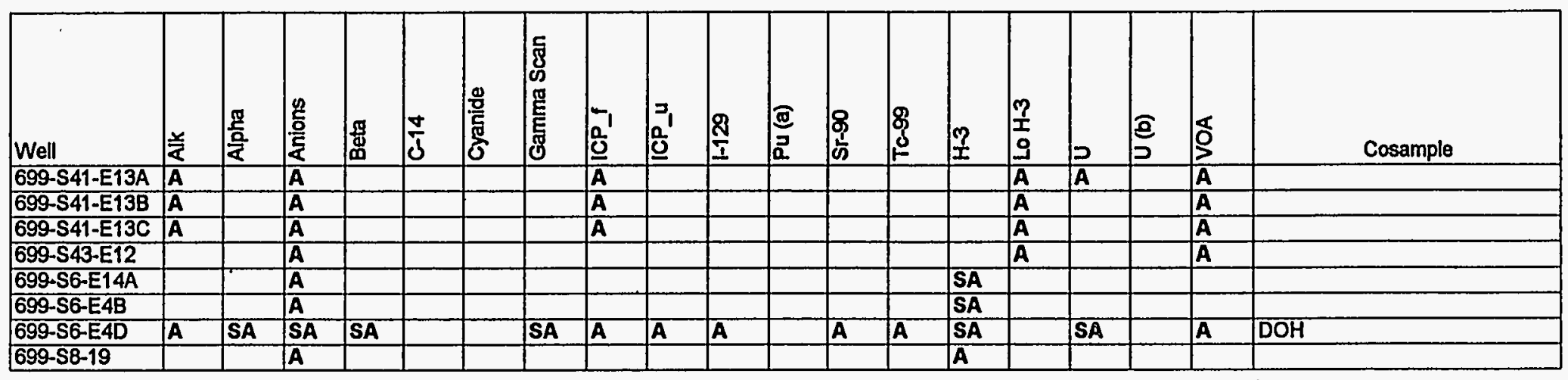

(a) Isotopic plutonlum.

(b) Isotopic uranlum. 


\subsection{WELL DISTRIBUTION ACCORDING TO PROGRAM}

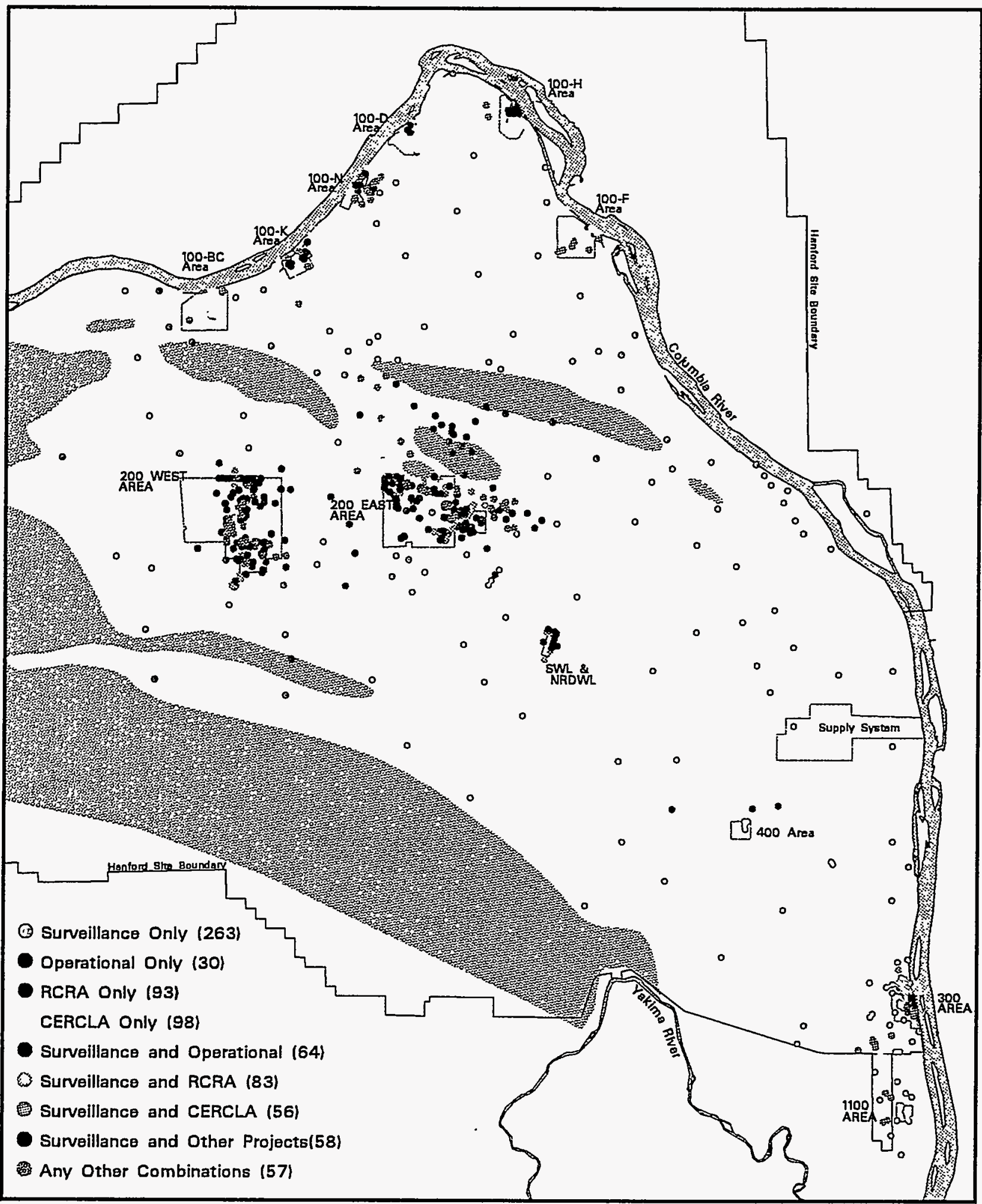

Figure 2.1. Distribution of Wel1s Sampled by the Ground-Water Surveillance, CERCLA, RCRA/Operational Monitoring Programs 


\section{DISTRIBUTION}

No. of

Copies

OFFSITE

12 DOE/Office of Scientific and Technical Information

R. Chong

U.S. Army Corps of Engineers Walla Walla District

Walla Walla Regional Airport, B1dg. 618

Walla Walla, WA 99362-9265

C. E. Cline

Washington State Dept. of Ecology 99 South Sound Center, MS 7600

Olympia, WA 98504-7600

R. Allan Danielson

Division of Radiation Protection Washington State Dept. of Health 1801 South 66th Ave

Yakima, WA 98808

J. L. Erickson

Division of Radiation Protection Radiation Control Section Washington State Dept. of Health Airindustrial Center, B7dg. 5, MS LE-13

$07 y m p i a$, WA 98504

R. E. Fink

U.S. Army Corps of. Engineers

Walla Walla District

Walla.Walla Regional Airport, Bidg. 618

Walla Walla, WA 99362-9265

Dave Jansen

Washington State Dept. of Ecology 99 South Sound Center, MS 7600

01ympia, WA 98504-7600
No. of

Copies

K. Kowalic

Washington State Dept. of Ecology

99 South Sound Center, MS 7600

01ympia, WA 98504-7600

3 Dave Nilander

Washington State Dept. of Ecology 7601 West Clearwater Street

Suite 102

Kennewick, WA 99336

R. D. Paris

Oregon Health Division

Radiation Control Section

Suite 705, 800 NE Oregon \#21

Portland, OR 97232

2 D. R. Sherwood

U.S. Environmental Protection Agency

P.0. Box 550, MS B5-01

Richland, WA 99352

9 Community-Operated Environmental.

Station Managers

L. D. Delitt

312 S. Columbia Center Blvd.

Kennewick, WA 99336

T. Droppo

3621 W. Sylvester

Pasco, WA 99301

D. R. Johns

904 Abbot Street

Richland, WA 99352

M. P. Madison

1715 N. 18th Avenue

Pasco, WA 99301 
No. of

Copies

J. $\mathrm{O}^{\prime} \mathrm{Nei} 11$

P. 0. Box 255

Umatilla, OR 97882

C. L. Stevenson

70 Canal Drive

Mesa, WA 99343

K. A. Thomas

6521 W. Argent

Pasco, WA 99301

C. A. Wagner

1528 Hunt Avenue

Richland, WA 99352

C. Zwiener

Rt. 2, Box 21504 A

Benton City, WA 99320

\section{ONSITE}

15 DOE Richland Operations Office

M. J. Furman, S7-55

J. D. Goodenough, H4-83

J. B. Hal1, A5-55

R. D. Hildebrand, A5-55

R. G. Holt, A5-15

R. G. McLeod, H4-83

H. P. Mooers, A5-55

P. M. Pak, H4-83

R. K. Stewart, H4-83

K. M. Thompson, H4-83

D. C. Ward (5), A5-55

2 U.S. Army Corps of Engineers

W. L. Greenwald, A3-47

W. D. Perro, A3-47

14 Bechtel Hanford Incorporated

J. M. Ayres, H4-89

J. V. Borghese, H4-90
No. of

Copies

K. R. Fecht, H4-85

B. H. Ford, H4-85

L. C. Hulstrom, H6-01

G. L. Kasza, H6-04

G. G. Kelty, H6-06

W. J. McMahon, H4-90

D. L. Parker, H6-02

R. E. Peterson, H4-89

R. F. Raidl, H4-89

L. C. Swanson, H6-03

D. C. Weekes, H4-82

C. D. Wittreich, H6-02

9 Westinghouse Hanford Company

L. P. Diediker, T1-30

J. J. Dorian, H6-20

D. G. Horton, H6-06

V. G. Johnson, H6-06

J. W. Lindberg, H6-06

S. M. McKinney, T1-30

R. M. Mitche11, H6-04

C. J. Perkins, $\times 0-21$

J. W. Schmidt H6-20

87 Pacific Northwest Laboratory

M. E. A7marode, K5-49

E. J. Antonio, K6-61

L. L. Be7t, K5-49

L. E. Bisping (30), K6-61

M. L. B lanton, K6-61

M. A. Chamness, K9-48

S. F. Conley, K6-79

A. T. Cooper, K6-61

R. L. Dirkes, K6-61

P. E. Dresel (2), K6-96

A. W. Endres, P7-02

J. C. Evans, K6-96

J. J. Fix, P7-02

B. M. Gillespie, K6-61

R. W. Hanf, Jr., K6-61

L. W. Hankel, K5-49

J. D. Harrison, K5-49

P. S. Henry, K6-79

E. L. Hilty, K6-96 
No. of

Copies

R. E. Jaquish, K9-25

K. K. Large, K6-61

J. J. Lopez, K5-49

E. W. Lusty, K6-79

S. P. Luttre11 (10), K6-96

D. L. Mackliet, K5-49

A. M. Marschman, K5-49

D. A. Muel1er, K6-79

B. E. Opitz, K6-79

T. M. Poston, K6-61

G. W. Patton, K6-61

J. J. Reck, K5-49

J. T. Rieger, K6-96

S. E. Rowe, K4-13

D. R. Sauer, K6-79

F. A. Spane, K6-96

D. L. Stewart, K6-96

B. L. Tiller, K6-63

P. D. Thorne, K6-96

T. L. VanArsdale, K6-79

W. D. Webber, K6-96

Historical File--R. L. Dirkes

Publishing Coordination

Technical Report Files (5)

Routing

R. M. Ecker

M. J. Graham

P. M. Irving

S. A. Rawson

P. C. Hays (1ast) 


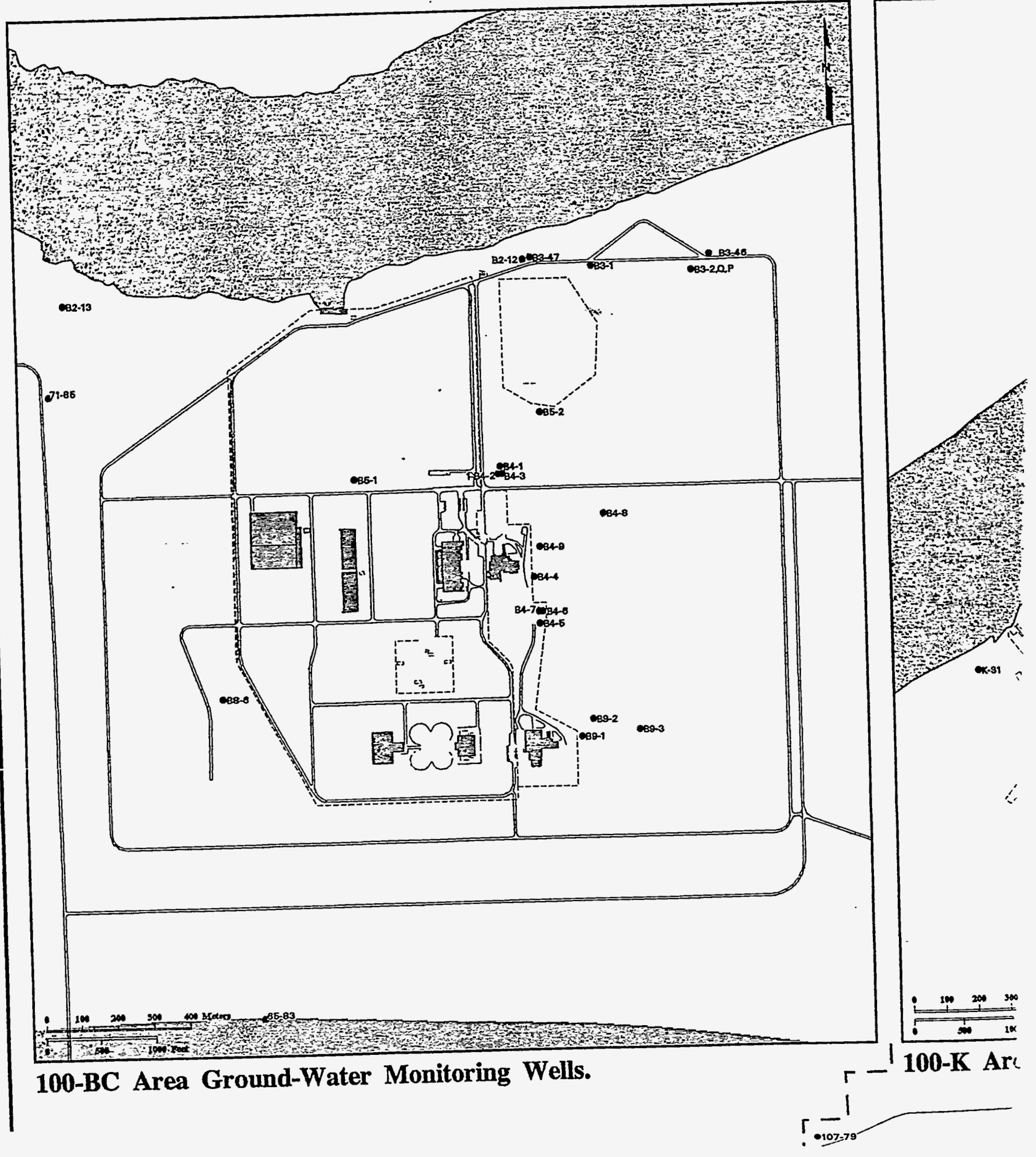




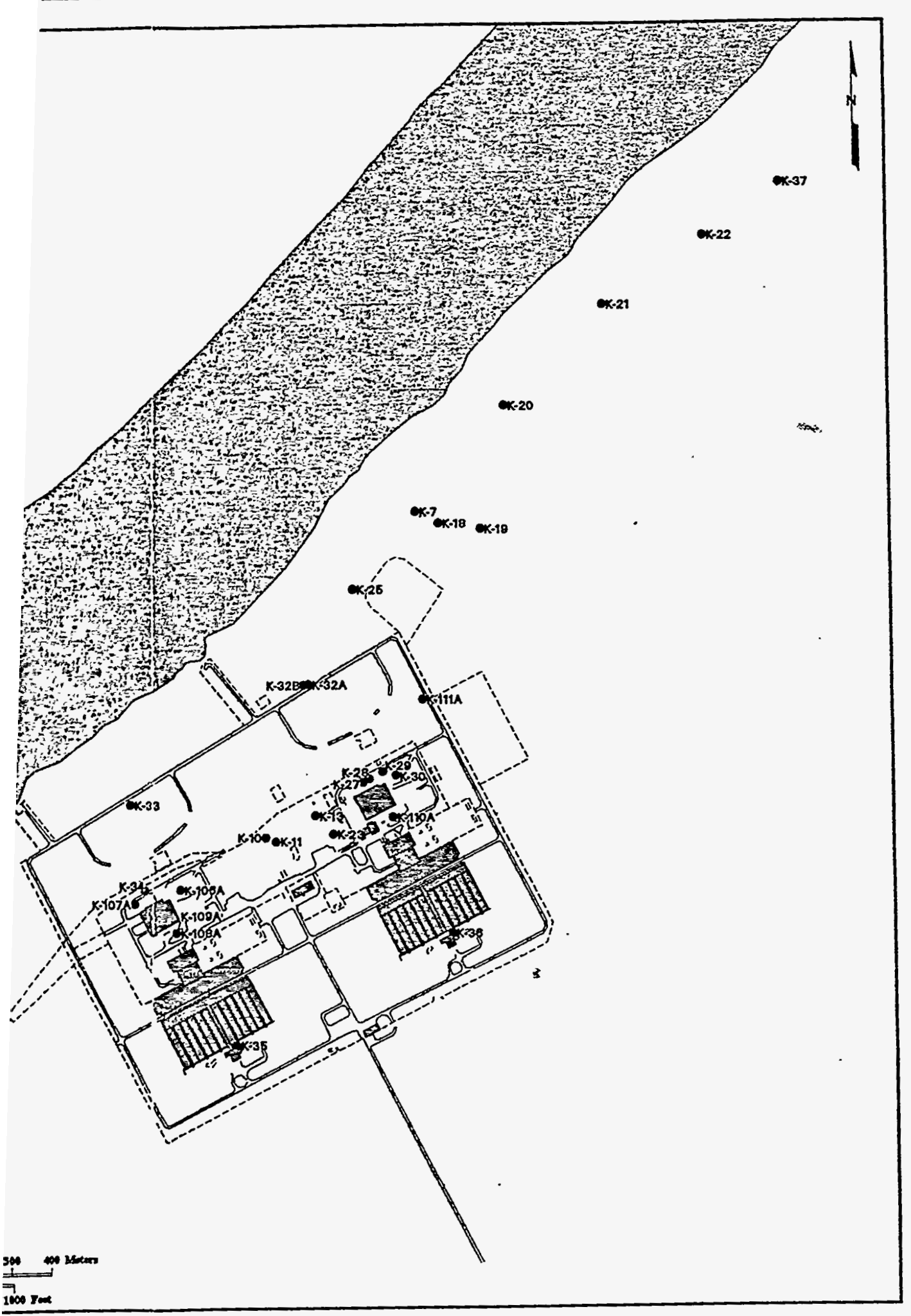

rea Ground-Water Monitoring Wells.

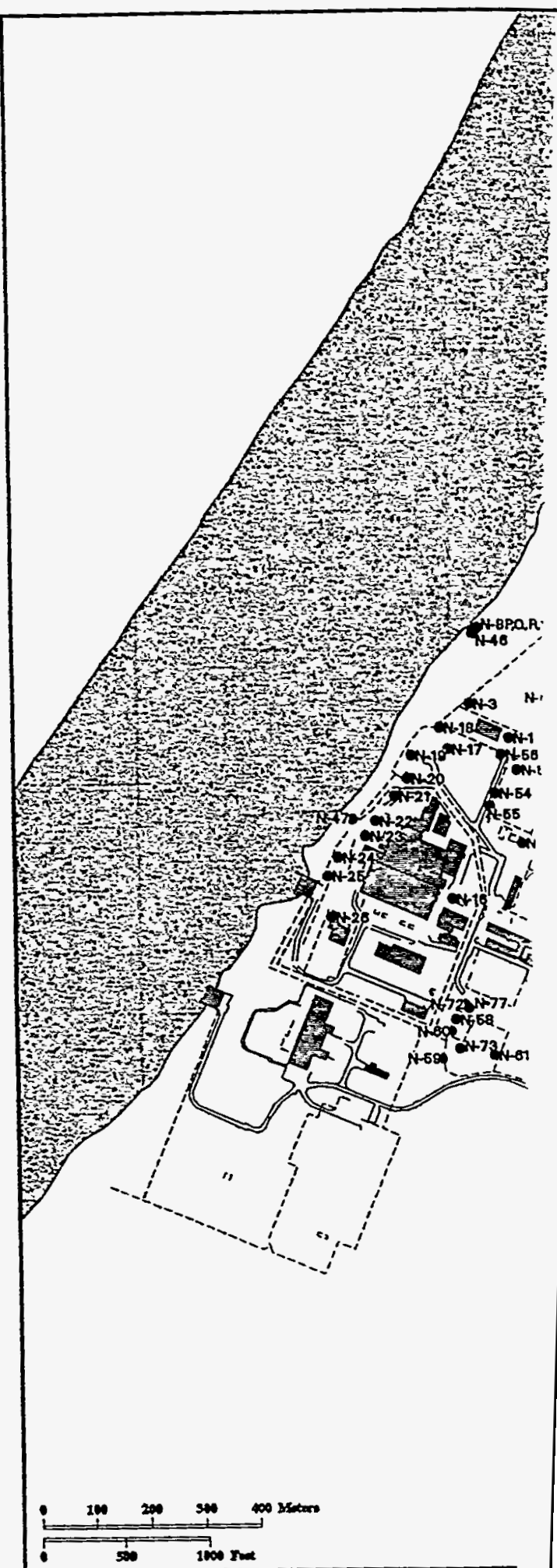

100-N Area Ground-Water :

Highway 24 


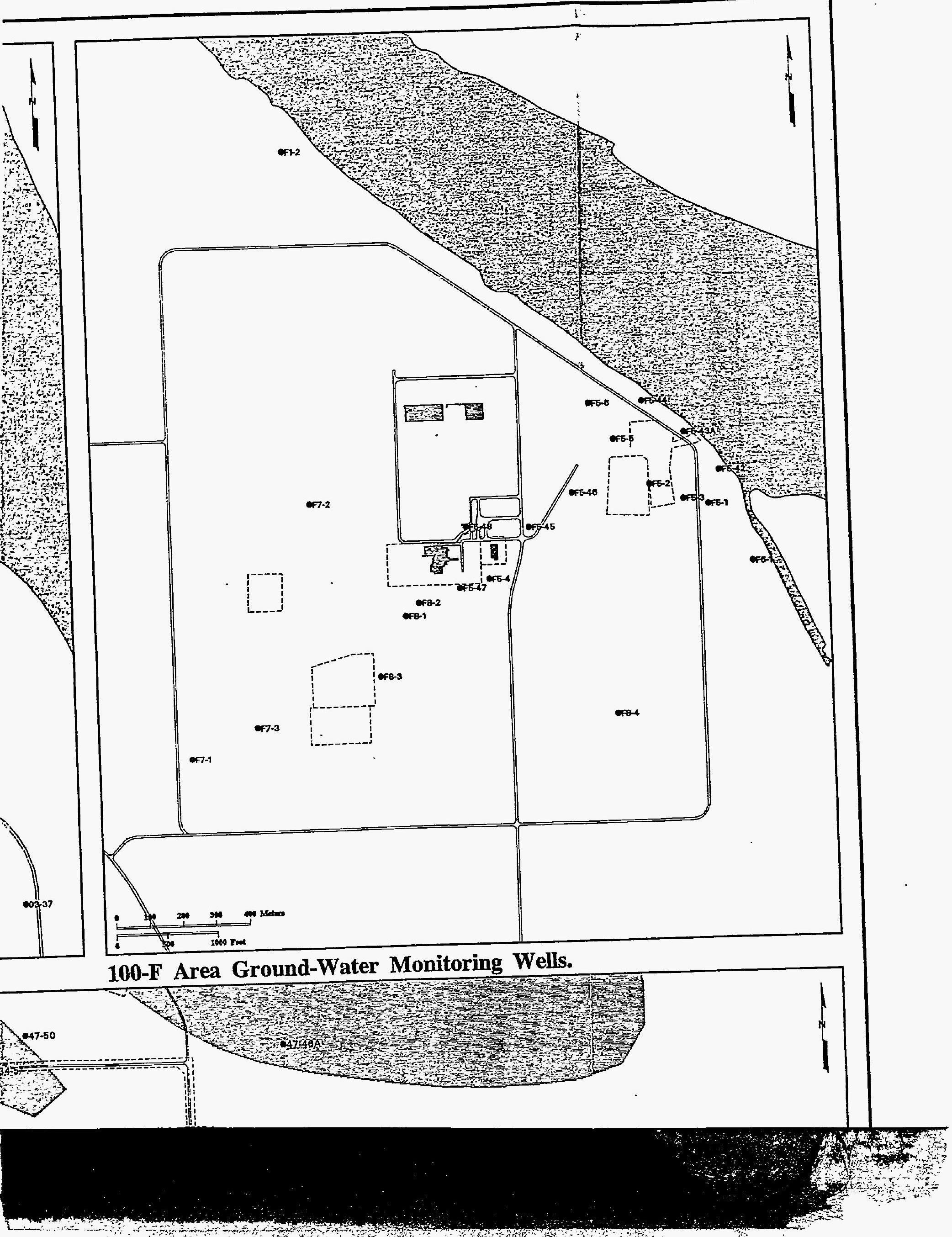




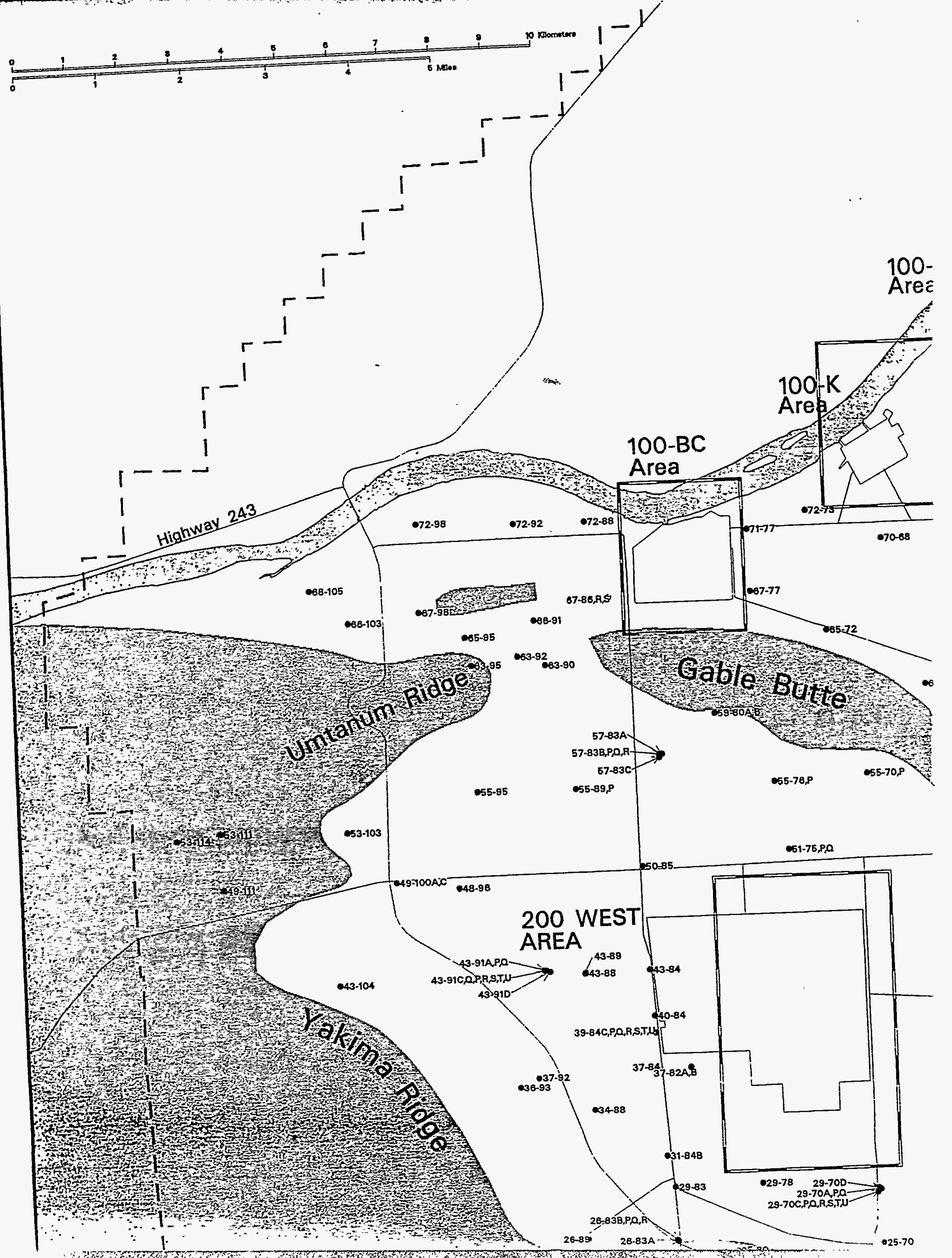




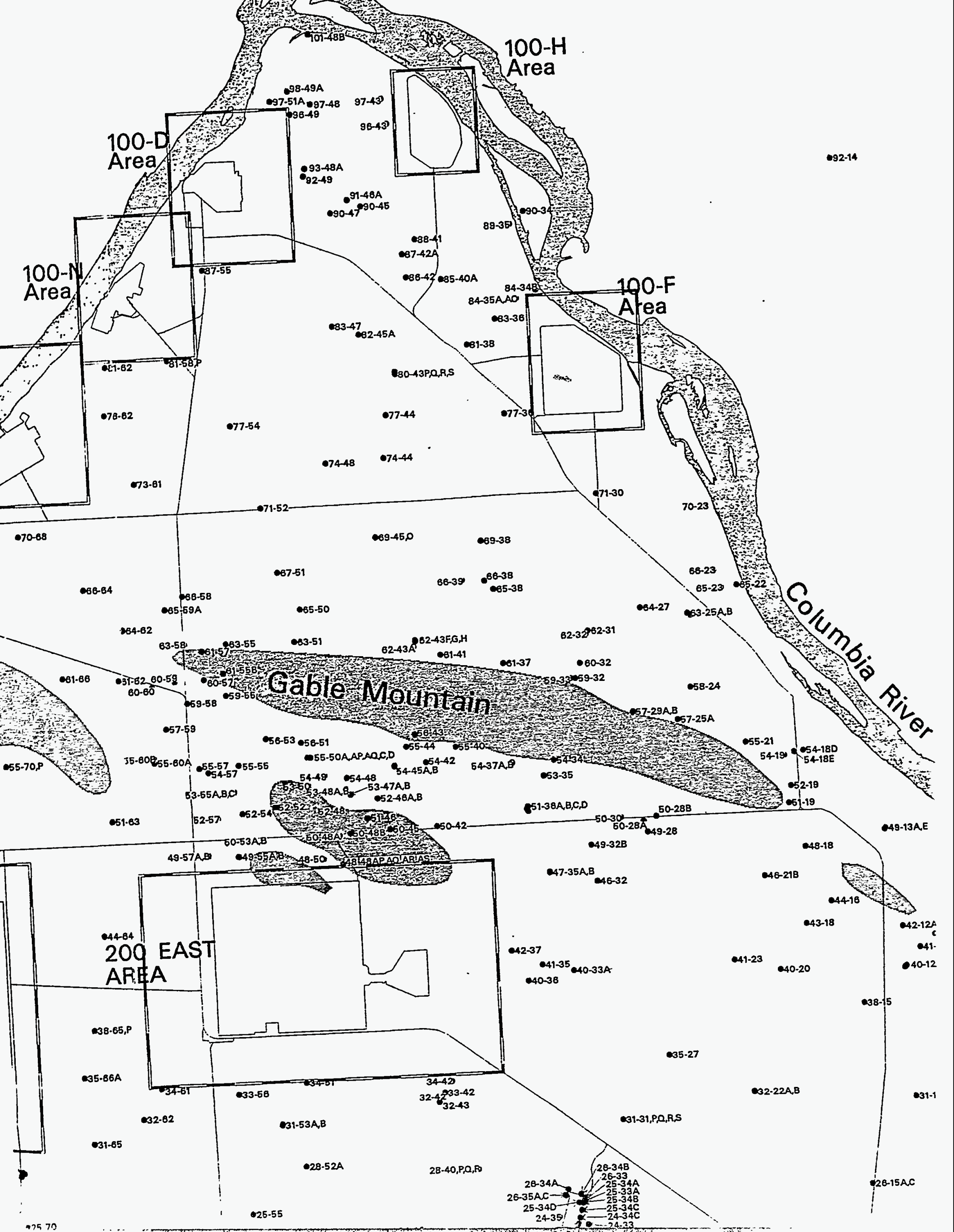




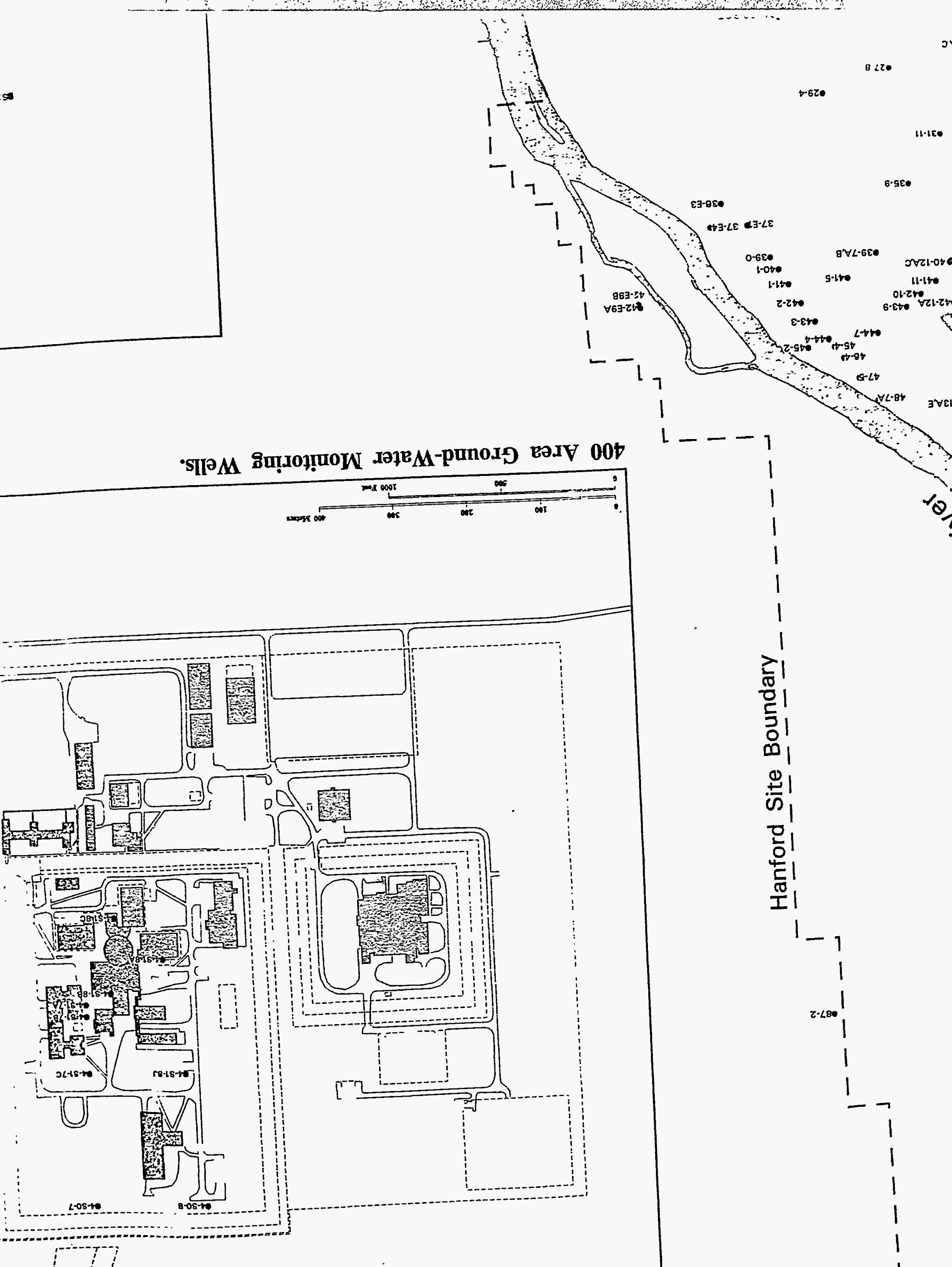




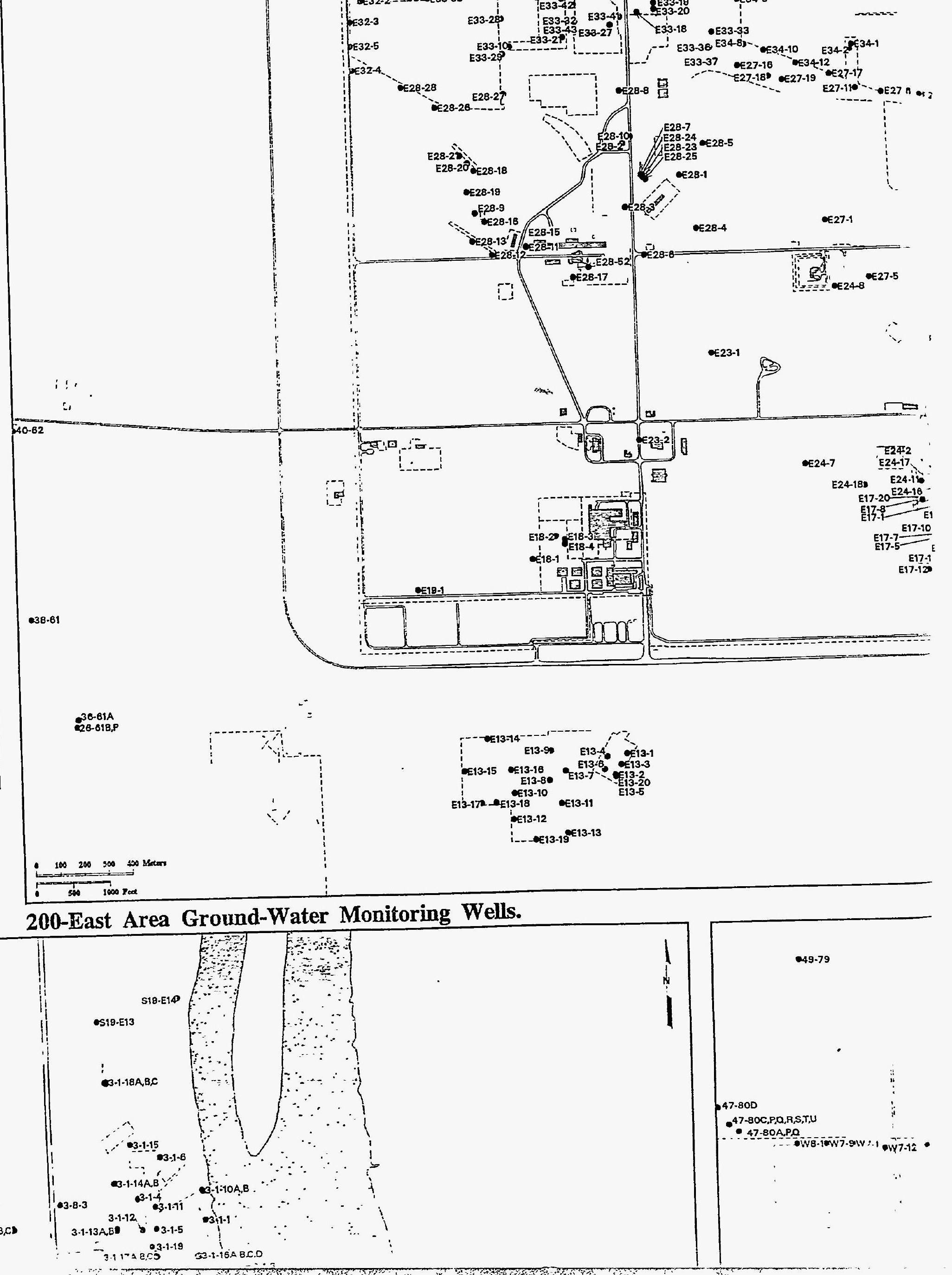




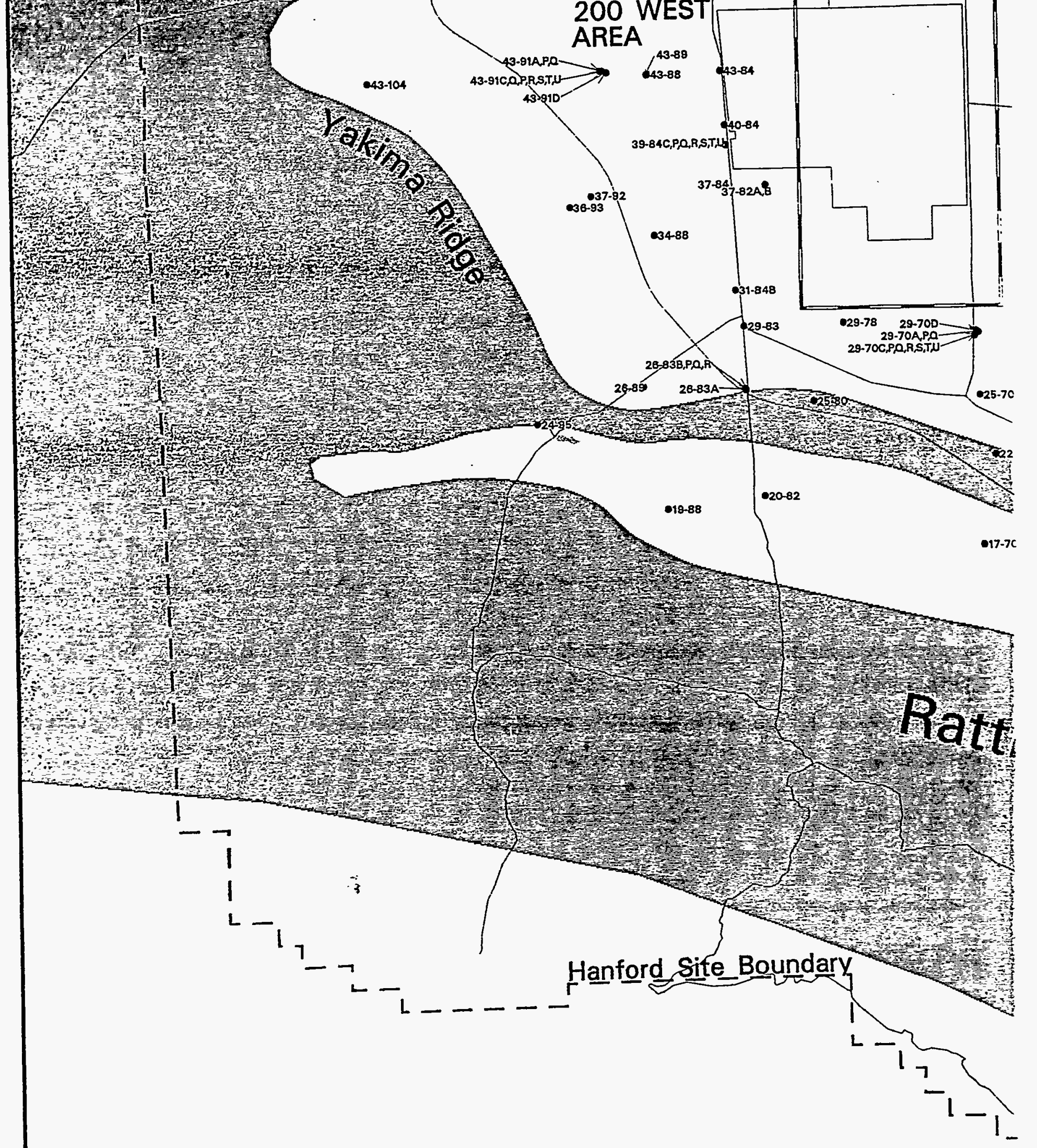




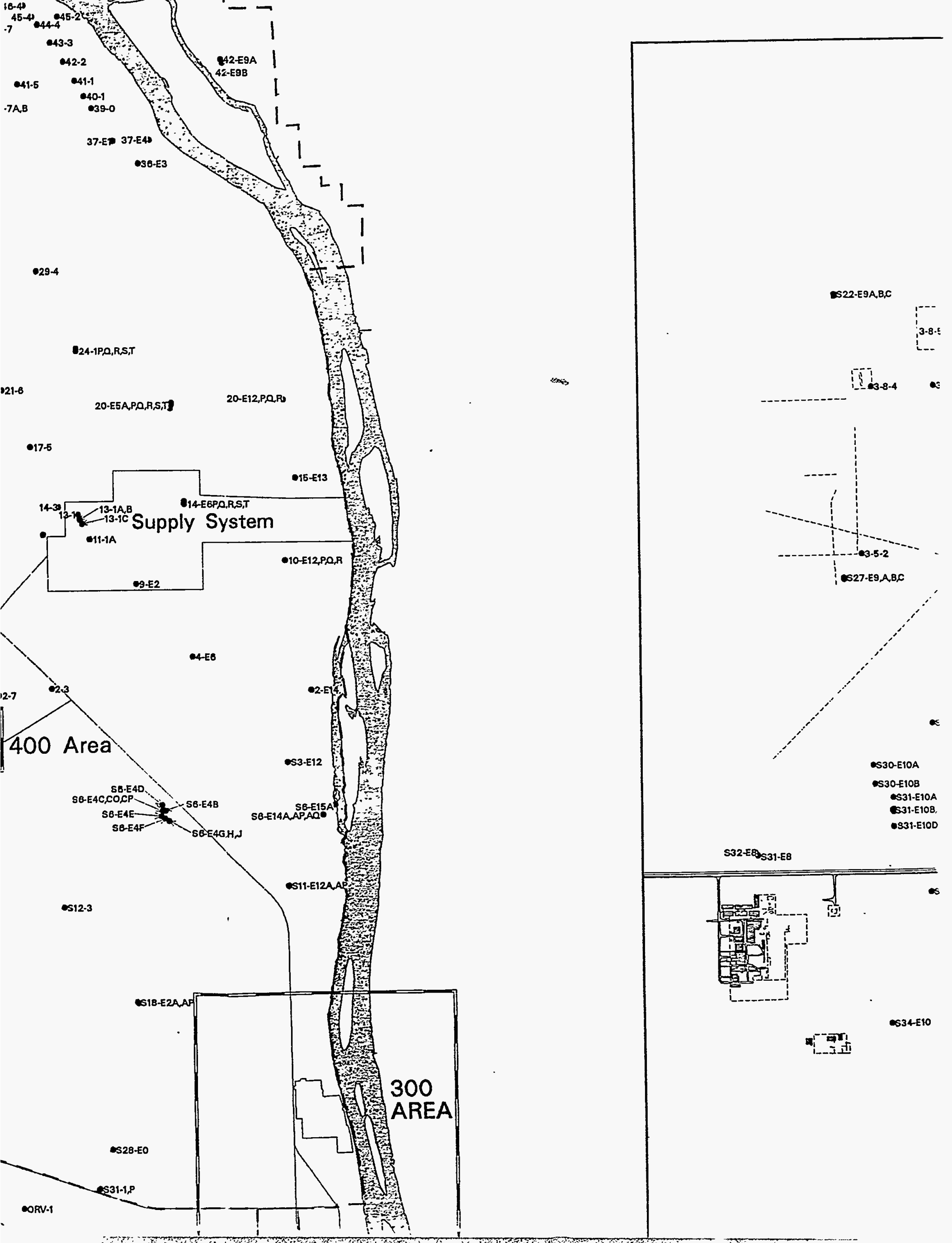




\section{0-East Area Ground-Water Monitoring Wells.}

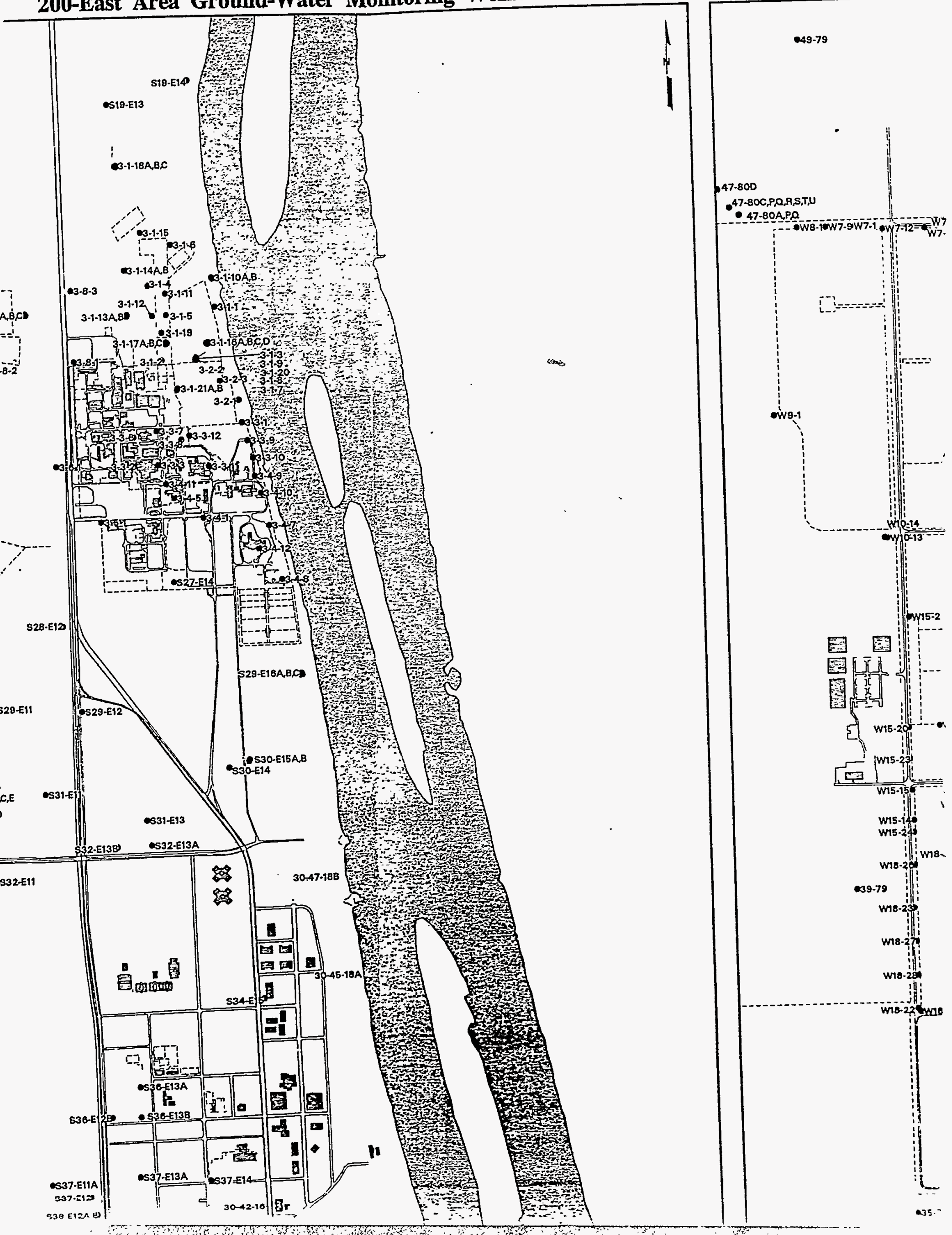




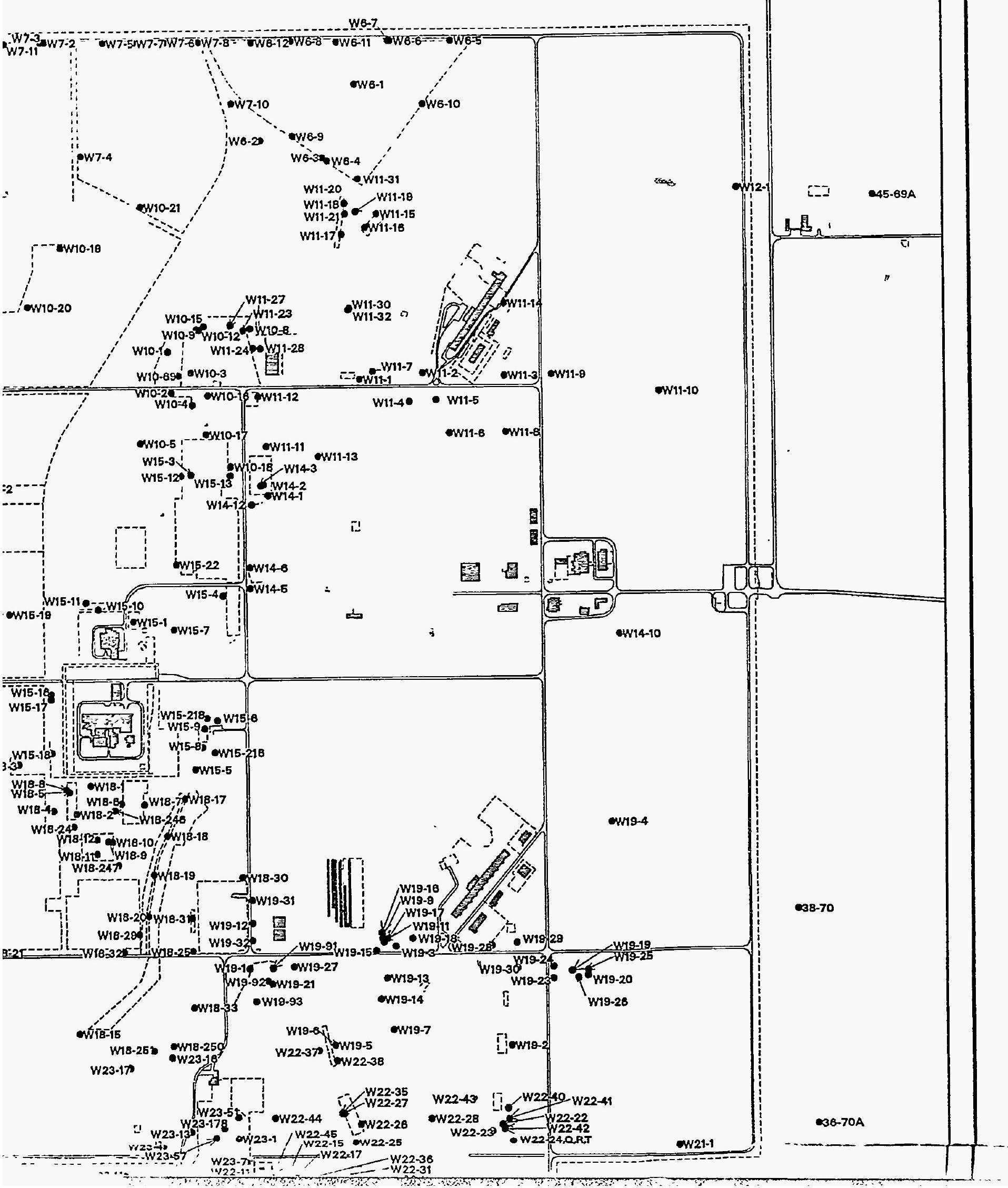


$\rightarrow$

\section{Plate1: Monitoring Wells At Th}




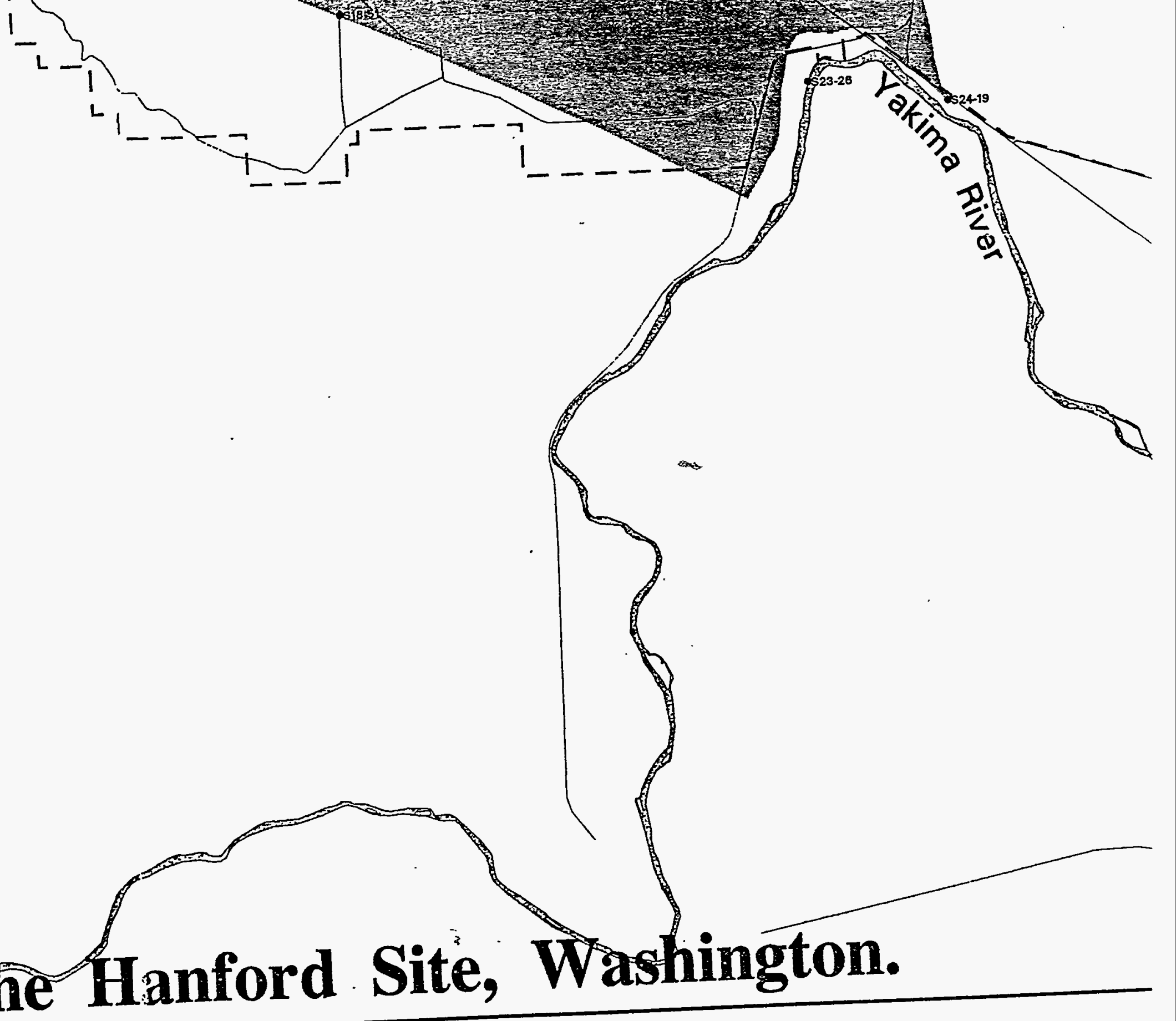




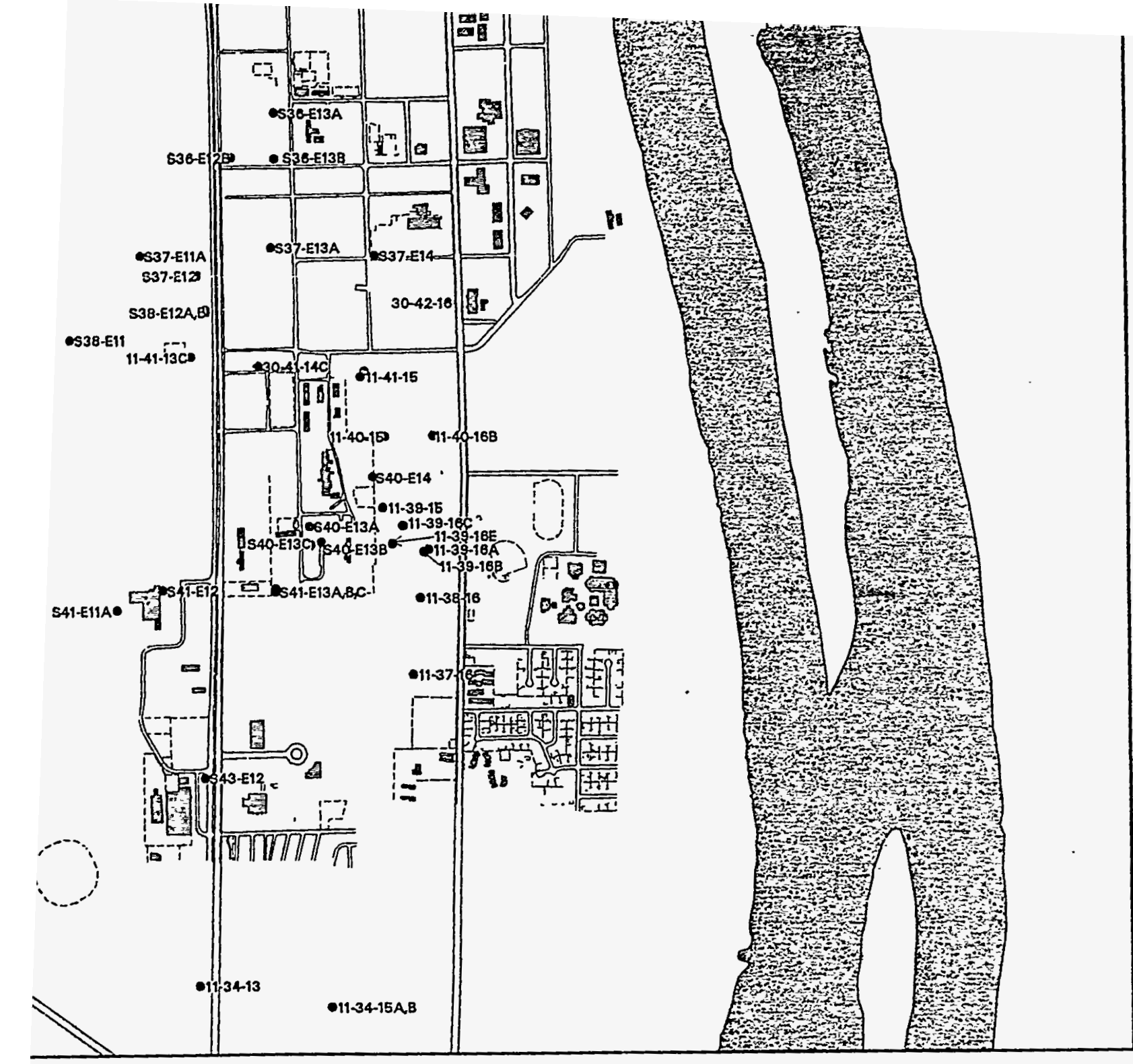

Area Ground-Water Monitoring Wells.

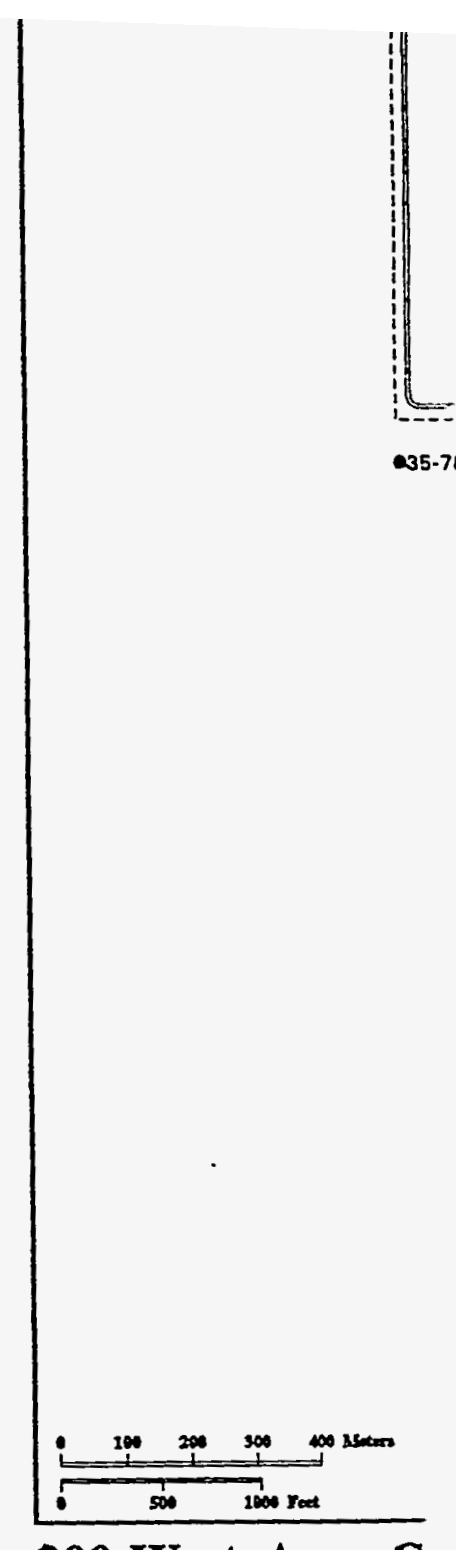

200-West Area G

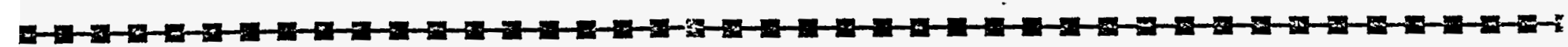




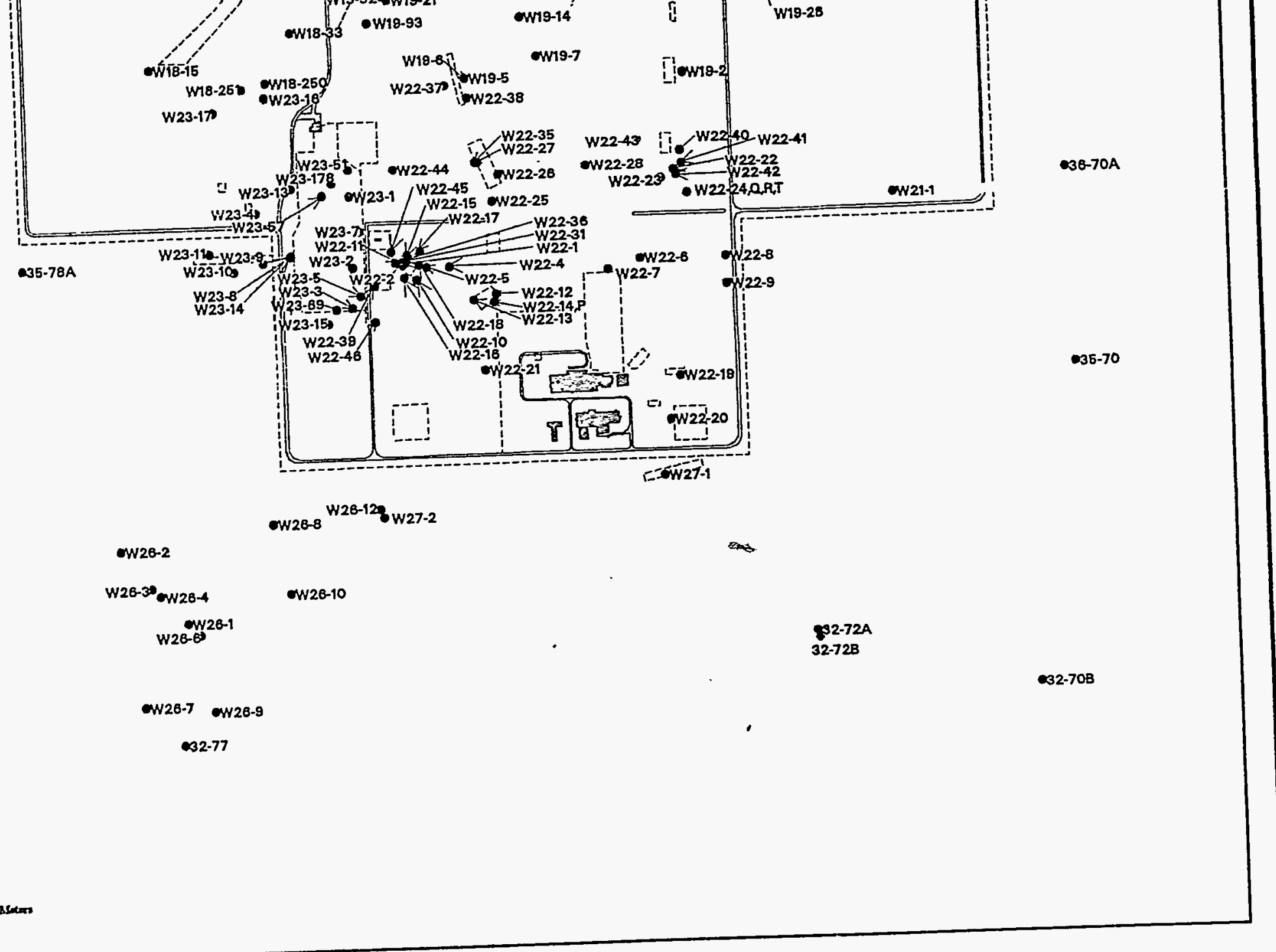

